\title{
Streamline-averaged mass transfer in a circulating drop
}

\author{
Paul Grassia ${ }^{\mathrm{a}, *}$, Sebastián Ubal ${ }^{\mathrm{b}}$ \\ ${ }^{a}$ Department of Chemical and Process Engineering, University of Strathclyde, \\ James Weir Building, 75 Montrose St, Glasgow G1 1 XJ, UK \\ ${ }^{b}$ UNL CONICET, INTEC, Güemes 3450, 3000 Santa Fe, Argentina
}

\begin{abstract}
Solute mass transfer is considered from the outside to the inside of a circulating drop in the context of liquid-liquid extraction. Specifically an internal problem is treated with resistance to mass transfer dominated by the liquid inside the drop. The Peclet number of the circulation is large, on the order of tens of thousands. A model is proposed by which the mass transfer into the drop begins in a boundary layer regime, but subsequently switches into a so called streamline-averaged regime. Solutions are developed for each regime, and also for the switch between them. These solutions are far easier to obtain than those of the full advection-diffusion equations governing this high Peclet number system, which are very stiff. During the boundary layer regime, the rate at which solute mass within the drop grows with time depends on Peclet number, with increases in Peclet number implying faster growth. However larger Peclet numbers also imply that the switch to the streamline-averaged regime happens sooner in time, and with less solute mass having been transferred to date. In the streamline-averaged regime, solute concentration varies across streamlines but not along them. In spite of the very large Peclet number, the rate of mass transfer is controlled diffusively, specifically by the rate of diffusion from streamline-to-streamline: sensitivity to the Peclet number is thereby lost. The model predictions capture, at least qualitatively, findings reported in literature for the evolution of the solute concentration in the drop obtained via full numerical simulation.

Keywords: Liquid-liquid extraction; Circulating drop; Mass transfer; High Peclet number; Streamline-averaged model; Mathematical modelling
\end{abstract}

\footnotetext{
*Corresponding author

Email address: paul.grassia@strath.ac.uk (Paul Grassia)
} 


\section{Highlights}

* Liquid-liquid extraction for a high Peclet number circulating drop is considered

* A boundary layer model for solute mass transfer is applied at early times

* A streamline-averaged model for solute mass transfer is applied at later times

* Boundary layer is switched to streamline-averaged model after one streamline orbit

* Solutions are obtained without a need to solve stiff advection-diffusion equations

\section{Introduction}

Liquid-liquid extraction is a versatile chemical engineering separation technique applicable in diverse fields, including metals processing (Jyothi et al., 2009; Nishihama et al., 2001), oil processing (Yahaya et al., 2013), biomolecule processing (Mazzola et al., 2008; Silva and Franco, 2000) and food processing (Moreno-Gonzalez and Garcia-Campana, 2017). The separation is realised (Richardson et al., 2002) via diffusive transfer of a solute dissolved in one solvent to another immiscible solvent down a gradient of chemical potential. Usually extraction proceeds by dispersing drops of the first solvent (dispersed phase) in the second one (continuous phase) and mass transfer either occurs into the drop (in the event that the solute is initially in the continuous phase) or out of the drop (if the solute is initially in the dispersed phase).

Although the extraction process actually involves a multitude of droplets contained within an extraction column (Mohanty, 2000), so as to understand what is happening at a fundamental level, a starting point is to consider a single drop (Wegener et al., 2014). Mass transfer to and/or from individual drops has been a widely studied topic in chemical engineering (Brodkorb et al., 2003; Handlos and Baron, 1957; Johns and Beckmann, 1966; Korchinsky et al., 2009; Kumar and Hartland, 1999; Negri and Korchinsky, 1986; Negri et al., 1986; Piarah et al., 2001; Ubal et al., 2011; Waheed et al., 2002), and the field becomes wider still if one accounts for analogous systems including heat transfer to/from drops (see e.g. Prakash and Sirignano (1978); Sadhal et al. (1997); Sirignano (2010)) as well as mass transfer to/from bubbles (see e.g. Juncu $(2005,2011)$ ).

In the case of liquid-liquid extraction, regardless of the direction of mass transfer 
(whether to or from the drop), it is useful to be able to predict how long the mass transfer process takes. This determines the residence time for which drops need to be present in an extraction column. Since drops will migrate through such a column at a speed determined (Wegener et al., 2014) by a balance between buoyancy and viscous drag forces, the residence time needed for mass transfer determines the required column height. Estimating the mass transfer time scale accurately is thereby important. If the estimate of the time scale required to achieve mass transfer is too low, then one is at risk of designing an extraction column that is too short, and hence that does not attain the target amount of mass to be transferred. By contrast, if the estimate of the time scale required to achieve mass transfer is too high, a column is likely to be over-designed to be taller than it needs to be, with higher cost implications.

As mentioned above, the driving force for liquid-liquid extraction is diffusive, i.e. transport down a gradient of chemical potential. However estimating the mass transfer time scale can be complicated by convective effects (Ruckenstein, 1967; Uribe-Ramirez and Korchinsky, 2000a,b). A drop of one solvent experiences shear stresses as it migrates through another immiscible solvent. These shear stresses set up fluid flow past the drop and a circulation pattern within the drop itself. Liquid-liquid extraction is thereby a convective-diffusive process rather than just a purely diffusive one.

Convection is beneficial to the extraction process (Ubal et al., 2010; Uribe-Ramirez and Korchinsky, 2000b) and the more complex the flow pattern is, the more beneficial convection tends to be (Edelmann et al., 2017). In the case, for instance, of transfer of a solute from the outside of a drop to the inside, the fluid flow past the drop (see Figure 1(a)) ensures that the drop surface is continually exposed to a new source of solute (rather than the solute concentration immediately outside the drop starting to become depleted, as would happen in the absence of any fluid flow). Moreover the circulation pattern inside the drop (again see Figure 1(a)) ensures that material which was originally near the drop surface (and which has therefore acquired solute from the outside by diffusion) is removed from the drop surface and replaced by fresh material (of low solute concentration) from the drop interior: this can keep solute concentration gradients confined to a sharp boundary 
layer (Uribe-Ramirez and Korchinsky, 2000b) near the drop surface (see Figure 1(b)), hence speeding up the rate of mass transfer quite substantially, although that situation does not necessarily last indefinitely (for reasons to be explained shortly).

Despite the evident benefits to the liquid-liquid extraction process, the presence of circulation complicates the computations that must be performed to determine exactly how liquid-liquid extraction proceeds. Although convection-diffusion equations are conceptually simple to set up (including being amenable to solution via commercial software packages) a significant issue in this particular system is that the circulation is usually rapid compared to diffusion (i.e. the relevant Peclet number is much larger than unity, often on the order of tens of thousands (Uribe-Ramirez and Korchinsky, 2000a,b)). This means that fluid must circulate around the drop many times before the extraction process is complete. Numerical simulations of the process, whilst possible (Edelmann et al., 2017; Ubal et al., 2011), are computationally very expensive owing to the need to resolve each individual circulation: convective-diffusive problems at high Peclet number are, in numerical terms, exceedingly stiff (Press et al., 1992).

A way to understand liquid-liquid extraction in circulating drops has been considered by Ubal et al. (2010) based on ideas originally proposed by Kronig and Brink (1950) and by Abramzon and Borde (1980); Brignell (1975); Oliver et al. (1985); Prakash and Sirignano (1978). Convection is, by definition, along streamlines, so that (at least in a system with steady and laminar fluid flow) the only way to transport material across streamlines is via diffusion, no matter how fast the flow. Since in high Peclet number flows, convection along streamlines is much faster than diffusion across them, it is expected (Ubal et al., 2010) that the solute concentration field should very rapidly become uniform along streamlines, but with diffusion-driven concentration variations from streamline to streamline (see Figure 1(c)). This then constitutes a streamline-averaged formulation of liquid-liquid extraction. Even though the mass transport rate into the drop is ultimately diffusively controlled, a benefit is still derived from convection. In order to fill the entire drop, the distance over which solute must diffuse is no longer the entire drop radius (as it would be in a system with no convection whatsoever (Korchinsky et al., 2009; 
Negri and Korchinsky, 1986; Negri et al., 1986)), but rather the distance from the drop surface to an internal stagnation point about which all the other streamlines circulate.

The exact location of that stagnation point depends on the exact flow field in the drop, but an earlier study using a plausible circulation pattern (Uribe-Ramirez and Korchinsky, 2000b) found it to be roughly one third of the drop radius beneath the surface. Since the time scale for diffusive processes is sensitive to distance (as can be established on dimensional grounds), convective-diffusive liquid-liquid extraction should proceed over a substantially shorter time scale than a comparable process without any convection. This has been verified by Ubal et al. (2010) using full numerical simulations of the convectivediffusive mass transfer process.

For the reasons pointed out above however (i.e. the Peclet number is very large (UribeRamirez and Korchinsky, 2000a,b) and so the governing equations are stiff), these numerical simulations proved extremely expensive. It is expected that a streamline-averaged theory will be much more amenable computationally, as it does not need to resolve the (very short) timescale associated with individual streamline circulations, but instead can focus exclusively on the longer diffusive time-scale over which mass transfer actually occurs. The necessary equations were in fact formulated by Ubal et al. (2010) but the solution was not implemented in that work, albeit there are previous implementations in literature for very a specific streamline layout (Brignell, 1975; Kronig and Brink, 1950; Prakash and Sirignano, 1978). Our purpose here is to revisit the implementation of the streamline-averaged theory, and to analyse the predictions it makes.

Solving the streamline-averaged theory is not however without difficulties. The theory assumes that solute concentration is uniform or near uniform along streamlines. This assumption is however invalid early on in the evolution (Ruckenstein, 1967; Uribe-Ramirez and Korchinsky, 2000b) for reasons we now explain. Consider for instance the situation as described earlier whereby solute diffuses into the drop from the outside. Circulating streamlines inside the drop that pass very near to the drop surface (and which are therefore exposed to the solute external to the drop) can and do acquire mass diffusively (UribeRamirez and Korchinsky, 2000b), but those same streamlines also penetrate very close 
to the drop axis, deep inside the drop where solute may not yet have reached. Until these streamlines undergo one complete circulation then (with material elements on the streamline having had the opportunity to pass along both the drop surface and the drop axis), it is a poor approximation to say the solute concentration is uniform along them. Instead what is required is an early-time theory valid up until the time of one complete circulation. The early-time theory must keep proper account of the solute mass entering the drop during this stage, so that the streamline-averaged theory which follows on from it is taken to start off with the correct amount of solute mass.

Suitable early-time theories have been proposed by Levich et al. (1965); Ruckenstein (1967); Uribe-Ramirez and Korchinsky (2000b); Vorotilin et al. (1965), and have also been discussed by Ubal et al. (2010). They fall into the general class of boundary layer theories (Leal, 2007) since at early times only streamlines passing near the drop surface acquire solute from the outside (see Figure 1(b)), those same streamlines subsequently being the ones that transport this solute along the drop axis (again see Figure 1(b)). Our secondary aim in this paper is to match the early-time boundary layer theory with the later time streamline-averaged theory: exactly how and when to switch between these theories has not been established previously.

Interestingly the work of Uribe-Ramirez and Korchinsky (2000b) treated an extension of the boundary layer theory which assumed that the solute acquired by streamlines as they passed close to the drop surface could subsequently be well mixed in the drop interior when carried along the parts of those same streamlines that passed close to the drop axis. This implied that when those same streamlines arrived back at the drop surface, they did so (by assumption) with a solute concentration far lower than the one with which they had formerly departed from it, having left behind a substantial amount of mass in the drop interior. Solute concentration gradients for fluid elements arriving back at the surface were thereby kept artificially large (Uribe-Ramirez and Korchinsky, 2000b), and the consequent predicted rate of mass transfer into the drop was likewise far too large.

Full numerical simulation of the (exceedingly stiff, and hence numerically expensive) convection-diffusion equations revealed that this assumption of solute mixing between 
streamlines within the drop interior was not valid (Ubal et al., 2010). Instead, for a streamline passing both very near the drop surface and very near the drop axis, the only material elements on that streamline which could arrive at the surface with low solute concentrations were those that had not yet been in close contact with the surface. Such material elements can however only survive up to one entire orbit of a streamline, implying that significant non-uniformities in solute concentrations along streamlines can likewise only survive that long. A further implication was that the large cross-stream concentration gradients near the drop surface which are predicted by the boundary layer theory (and the rapid mass transfer into the drop that these large gradients imply) cannot last indefinitely, but rather only up to the time at which one entire streamline orbit is complete (as indeed the work of Brignell (1975) recognised). The conclusion of Ubal et al. (2010) then was not that the boundary layer theory itself was inherently incorrect, merely that it was inappropriate to apply it for times far beyond a single orbit time.

To reiterate, once an entire streamline orbit is complete, all elements on the streamline in question must have spent part of their life near the surface (acquiring solute from the exterior of the drop) and part of their life near the drop axis (but barely mixing solute with other streamlines). This means that, not only do gradients in solute concentration normal to the drop surface (and hence mass transfer rates into the drop) start to decrease from one orbit time onwards, the solute concentration on the entire near surface streamline should be comparatively uniform, being set by the concentration immediately outside the drop. This however is the criterion for the previously mentioned streamline-averaged theory (Ubal et al., 2010) to begin to apply. In other words the upper time limit for applicability of the early-time boundary layer theory should coincide with the lower time limit for applicability of the streamline-averaged theory.

It is plausible then that the entire evolution of the mass transfer process in a circulating drop can be described by selecting a suitable time to switch between the two aforementioned theories. This then is the hypothesis that we investigate here. Moreover, as we will discuss shortly, one of the features of the streamline-averaged theory is that it can be set up retaining just very incomplete information about the streamline pattern, 
rather than requiring complete knowledge of all details of the geometry and kinematics of the flow field in the drop. Thus one of the key questions we shall consider is whether it is feasible to predict evolution of concentration fields in the drop using just incomplete information about the circulation.

This work is laid out as follows. Section 2 discusses the equations governing convectivediffusive mass transfer within a drop during liquid-liquid extraction, and how these reduce to either boundary layer theories or streamline-averaged theories in relevant limits. Section 3 details the methods used to solve the equations governing each of the above mentioned theories, as well as the parameter values selected in each case. After that, section 4 presents results obtained for each theory and the process of switching between them. Section 5 presents conclusions.

\section{Theory and governing equations}

This section is laid out as follows. The general equations for mass transfer into a circulating liquid drop are given in section 2.1. A boundary layer theory of the mass transfer process is described in section 2.2, building upon material which is already presented in Ubal et al. (2010). Streamline orbit times are computed in section 2.3: these are needed in order to determine the time to switch from a boundary layer theory to a streamline-averaged one. As we will see, defining this time scale which nominally corresponds to "one streamline orbit time" is far from trivial. The streamline-averaged theory itself is presented in section 2.4, again building on material already presented in Ubal et al. (2010) and even earlier papers (Brignell, 1975; Kronig and Brink, 1950; Prakash and Sirignano, 1978). Boundary and initial conditions for solving the streamline-averaged system however turn out to be non-trivial, and these are considered in section 2.5. An overall summary of the theory is given in section 2.6.

\subsection{Convective-diffusive mass transfer}

We consider a solvent drop of radius $R$, moving through an immiscible solvent at speed $U$ : see Figure 1(a). For simplicity, we envisage that the drop motion is driven by buoyancy 
forces, and assume that it is moving vertically upwards (although the theory presented here is not affected by the nature of the driving force and/or direction of motion).

The drop is assumed to remain spherical as it moves and, in the frame of reference of the drop, a spherical polar coordinate system denoted $r^{\prime}, \theta, \phi$ is defined. Circulation develops in the drop whilst fluid flows past outside and (still in the drop's reference frame) we define a fluid velocity field $\boldsymbol{u}^{\prime}$. Although quite complex flow patterns can arise (Edelmann et al., 2017), for simplicity, $\boldsymbol{u}^{\prime}$ is assumed to be steady state and axisymmetric, so that velocity components $u_{r}^{\prime}$ and $u_{\theta}^{\prime}$ depend on $r^{\prime}$ and $\theta$, but not on $\phi$, and moreover $u_{\phi}^{\prime}$ vanishes. The values of $u_{r}^{\prime}$ and $u_{\theta}^{\prime}$ can be computed in principle given $R, U$, and given the viscosities and densities outside and inside the drop, but for the present we do not specify exactly what these velocity fields are, focussing instead on mass transfer.

A solute is dissolved in the external solvent at some mass fraction $\tilde{w}_{R}$ whereas the solute is initially at mass fraction $w_{0}^{\prime}$ within the drop itself. The solute has a diffusivity $D$ within the drop, and possibly a different diffusivity outside the drop. We suppose that solute at mass fraction $\tilde{w}_{R}$ in the external solvent has the same chemical potential as solute at mass fraction $w_{R}^{\prime}$ in the internal solvent. We also suppose that $w_{R}^{\prime}$ exceeds $w_{0}^{\prime}$ so that the direction of mass transfer is into the drop (although the case where the direction of transfer is out of the drop is entirely analogous to what we present).

We define a drop Peclet number as

$$
P e=R U / D
$$

noting that in problems of interest this is typically on the order of tens of thousands (UribeRamirez and Korchinsky, 2000a,b). We are interested in solving for the time evolution of mass fraction represented by a field $w^{\prime}$ within the drop and $\tilde{w}$ outside it. We denote time by $t^{\prime}$, and the gradient operator by $\nabla^{\prime}$. We also define a dimensionless time by $t=t^{\prime} D / R^{2}$ (i.e. time made dimensionless on a diffusive time scale), a dimensionless coordinate by $r=r^{\prime} / R$, a dimensionless fluid flow field $\boldsymbol{u}=\boldsymbol{u}^{\prime} / U$ (with components $u_{r}$ and $u_{\theta}$ ) and a dimensionless gradient operator $\nabla=R \nabla^{\prime}$. In terms of these dimensionless variables, the 
governing convection-diffusion equation inside the drop is

$$
\partial w^{\prime} / \partial t+P e \boldsymbol{u} \cdot \nabla w^{\prime}=\nabla^{2} w^{\prime}
$$

The equation we must solve for $\tilde{w}$ outside the drop is broadly similar, requiring only a minor change to reflect the fact that the diffusivity outside the drop possibly differs from that inside. Although we could in principle solve a coupled mass transfer problem both inside and outside the drop, to simplify the system of equations, we shall suppose that the resistance to diffusive mass transfer is dominated by the inside of the drop, meaning we solve what is known as an internal problem, rather than a coupled one. At one level this can be viewed as equivalent to supposing that the solute diffusivity inside the drop is substantially lower than that outside. However even if the two diffusivities are comparable, assuming that resistance to diffusion is dominated by the inside of the drop is actually not unreasonable, at least over a significant period of the drop's evolution (Ubal et al., 2010). The fluid elements on the outside of the drop pass by the drop once only so have only a limited opportunity (and a limited time) to lose solute mass to the drop. The fluid elements on the inside however circulate around multiple times. The implication is that any gradients of mass fraction have only limited time to develop outside the drop (and hence develop over a comparatively small distance), but have a much longer time to develop inside the drop (and hence develop over longer distances). To compensate for this imbalance in distance scales, the change in mass fraction outside the drop tends to be rather smaller than that inside ${ }^{1}$. Hence by assumption we only solve an internal problem - equation $(2)$ - where the initial condition at $t=0$ is $w^{\prime}=w_{0}^{\prime}$ and the boundary condition at $r=1$ is $w^{\prime}=w_{R}^{\prime}$.

It is convenient to define a "normalised" or "rescaled" mass fraction denoted $w$ as

$$
w=\left(w^{\prime}-w_{0}^{\prime}\right) /\left(w_{R}^{\prime}-w_{0}^{\prime}\right)
$$

\footnotetext{
${ }^{1}$ Clearly there are exceptions to this general rule, e.g. the case of a gas bubble in liquid (Juncu, 2005, 2011), as opposed that of a liquid drop, where the diffusivity inside the bubble is far greater than that outside making the change in mass fraction inside the bubble become exceedingly small. Such cases are not however considered here.
} 
Equation (2), being linear in $w^{\prime}$, applies equally well to $w$ as to $w^{\prime}$, but writing it in terms of $w$ is convenient since $w$ varies across the full range of 0 to 1 , the initial condition being $w=0$ and the boundary condition being $w=1$. In what follows we will loosely refer to $w$ as a "mass fraction", although it must be remembered that the true mass fraction $w^{\prime}$ varies only between $w_{0}^{\prime}$ and $w_{R}^{\prime}$ with typically $w_{R}^{\prime}-w_{0}^{\prime}$ being much smaller than unity.

Equation (1) is challenging to solve not only because $P e$ is large (meaning the equation is stiff (Press et al., 1992)) but also because $\boldsymbol{u}$ can in general vary in a quite complicated way in space. One of the strategies for trying to solve equation (1) is to look at a boundary layer theory, which accounts for very different length scales in the cross-stream and streamwise direction, and is simplified by only needing to consider the values of $\boldsymbol{u}$ at the drop surface. This particular theory is discussed in the next section.

\subsection{Boundary layer theory}

The boundary layer theory for mass transfer from a circulating drop has been developed by Levich et al. (1965); Ruckenstein (1967); Uribe-Ramirez and Korchinsky (2000b); Vorotilin et al. (1965) but here we follow the development of Ubal et al. (2010) which included a discussion of how to interpret the boundary layer theory physically: readers familiar with the arguments of Ubal et al. (2010) may prefer to skip directly to section 2.3.

It is assumed that (at sufficiently early times) variations in (normalised) mass fraction $w$ are confined near the drop surface, or at least to streamlines that pass close to the drop surface. A (dimensionless) streamfunction $\psi$ is also defined such that

$$
\begin{aligned}
& u_{r}=\left(r^{2} \sin \theta\right)^{-1} \partial \psi / \partial \theta \\
& u_{\theta}=-(r \sin \theta)^{-1} \partial \psi / \partial r .
\end{aligned}
$$

There is a free additive constant in the streamfunction which is set here so that the streamline comprising the drop surface and drop axis has $\psi=0$. The sense of the circulation here is such that $\psi$ is positive inside the drop. Moreover the drop contains an internal stagnation point: the streamfunction has a local maximum at this point. 
A (dimensionless) time variable $T$ (referred to a convective time scale) is defined via

$$
T=t^{\prime} U / R \equiv P e t
$$

Clearly, since $P e \gg 1$, if we are interested in order unity values of $T$ (corresponding to time scales comparable with one streamline orbit time), this corresponds to times $t \ll 1$.

A Lagrangian derivative on the drop surface D/DT is defined as

$$
\mathrm{D} / \mathrm{D} T=\partial / \partial T+\left.u_{\theta}\right|_{\operatorname{surf}} \partial / \partial \theta
$$

where $\left.u_{\theta}\right|_{\text {surf }}$ is the velocity field along the drop surface ${ }^{2}$.

Additionally a so called extent of diffusion function $\zeta$, which is a function of polar angle $\theta$ and time $T$ (but not of radial coordinate $r$ ) is defined such that

$$
\mathrm{D} \zeta / \mathrm{D} T \equiv \partial \zeta / \partial T+\left.u_{\theta}\right|_{\mathrm{surf}} \partial \zeta / \partial \theta=\left.u_{\theta}^{2}\right|_{\text {surf }} \sin ^{2} \theta .
$$

Observe (via equation (5)) that $\mathrm{D} \zeta / \mathrm{D} T$ is the square of the cross-stream gradient of $\psi$ at the surface: hence the more closely spaced are the streamlines, the faster the rate at which $\zeta$ grows following a fluid element.

Equation (8) is to be solved with an initial condition $\zeta=0$ when $T=0$, and we also impose, at least for the moment, a boundary condition ${ }^{3}$ that $\zeta=0$ when $\theta=0$. The physical interpretation of $\zeta$ was explained by Ubal et al. (2010): it represents a "diffusive clock" which runs fast when streamlines are close together (making it easier to exchange mass between them) and which runs slow when streamlines are far apart (so that mass exchange becomes more difficult). Streamlines actually tend to move further apart both due to the kinematics (i.e. as the speed on surface decreases) and due to the geometry

\footnotetext{
${ }^{2}$ Note that $u_{r}$ vanishes on the drop surface because the drop is assumed to remain spherical, hence the surface velocity field only needs to be specified for $u_{\theta}$.

${ }^{3}$ This boundary condition, $\zeta=0$ for $\theta=0$, implies that any material newly arriving at the drop surface from the interior has not previously been on the drop surface, and so has not previously been able to exchange mass with the exterior of the drop. Since it turns out that we are only interested in using the boundary layer theory up to the order of one streamline orbit time, and not for times any longer than that, such a condition is actually reasonable.
} 
(i.e. as points on the surface move closer to the axis). In fact these two effects cooperate with one another because there are stagnation points where the drop axis intersects the drop surface (respectively a forward stagnation point and a rear stagnation point), and hence $\left.u_{\theta}\right|_{\text {surf }}$ is small whenever $\sin \theta$ is small.

In the remainder of this section we explain how to obtain the extent of diffusion (section 2.2.1), the solute mass fraction field (section 2.2.2), and the total amount of solute within the drop (sections 2.2.3-2.2.4).

\subsubsection{Solving for the extent of diffusion}

Equation (8) can be solved by first identifying a location $\theta_{0}$, a function of $\theta$ and $T$, defined as the initial angular location of a material element on the drop surface that subsequently happens to reach angular location $\theta$ at time $T$. This is easily defined implicitly

$$
T=\int_{\theta_{0}}^{\theta} \mathrm{d} \theta /\left.u_{\theta}\right|_{\text {surf }}
$$

At very early times, such that $T \ll 1$, it is evident that $\theta_{0}$ is just slightly smaller than $\theta$. For any given $\theta$, as $T$ increases, $\theta_{0}$ migrates back towards zero. Increasing $\theta$ however at any given $T$ also increases $\theta_{0}$.

Once $\theta_{0}$ is defined, the value of $\zeta$ then follows (Ubal et al., 2010)

$$
\zeta=\left.\int_{\theta_{0}}^{\theta} u_{\theta}\right|_{\operatorname{surf}} \sin ^{2} \theta \mathrm{d} \theta
$$

\subsubsection{Solving for the solute mass fraction field}

The solute mass fraction field can now be given (Ubal et al., 2010) in terms of the streamfunction $\psi$, Peclet number $P e$ and extent of diffusion $\zeta$

$$
w=\operatorname{erfc}(\psi \sqrt{P e} / \sqrt{4 \zeta})
$$

This has the same functional form for $w$ as employed by Uribe-Ramirez and Korchinsky (2000b), despite the predictions that we will obtain from it later on being very different from theirs. This underlines that the main issue with the work of Uribe-Ramirez and 
Korchinsky (2000b) was not the formulation of the boundary layer model itself, but rather the attempt to apply it out to times far longer than its true domain of applicability.

Note that equation (11) includes both variation across streamlines (the term in $\psi$ ) and along them (angular coordinate $\theta$ varies along near surface streamlines, and $\zeta$ varies with $\theta$, as well as with $T$ ). Despite this potentially complicated variation of $w$, it is clear that solute mass really is distributed in a boundary layer (see e.g. Figure 1(b)), since the only streamlines that have significant solute mass fraction are those with streamfunction $\psi$ smaller than order $\sqrt{\zeta / P e}$, where recall that $\psi$ vanishes on the drop surface (and so is small near the drop surface) and that $P e \gg 1$.

\subsubsection{Solving for the solute mass in the drop}

Equation (11) can be used to determine the total amount of solute mass that has entered the drop as follows. We define a coordinate $n$ to be distance of a point inside the drop from the drop surface, i.e. $n=1-r$. The solute mass $M$ that has entered the drop (or rather the "normalised" solute mass, in view of equation (3)) is then obtained via

$$
M=2 \pi \int_{0}^{\pi} \int_{0}^{1} w \sin \theta(1-n)^{2} \mathrm{~d} \theta \mathrm{d} n
$$

Although in principle $n$ can vary between 0 and 1 , in practice we are only interested in values of $n$ very close to the drop surface, since $w$ decays very rapidly away from the drop surface. This means we can approximate $1-n$ by unity. Near the drop surface, equation (5) implies moreover $\partial \psi /\left.\partial n \approx u_{\theta}\right|_{\operatorname{surf}} \sin \theta$. We can therefore change the integration variable in equation (12) from $n$ to $\psi$. We can also shift the upper limit of the $\psi$ integration to infinity, owing to the very rapid decay of $w$ (which effectively vanishes for any $\psi$ value in excess of order $\sqrt{\zeta / P e})$. Hence

$$
M=2 \pi \int_{0}^{\pi} \int_{0}^{\infty} w \mathrm{~d} \theta \mathrm{d} \psi /\left.u_{\theta}\right|_{\text {surf }}
$$

Substituting from equation (11) and integrating over $\psi$ gives

$$
M=2 \pi \int_{0}^{\pi} \sqrt{4 \zeta /(P e \pi)} \mathrm{d} \theta /\left.u_{\theta}\right|_{\text {surf }} .
$$


Although equation (14) gives a formal expression for the amount of solute mass that has entered the drop, it is not always convenient to use. As fluid elements migrate along the surface of the drop they collect more and more solute, or equivalently, solute reaches increasing numbers of streamlines. There is however a rear stagnation point at the downstream end of the drop, and in the neighbourhood of this point streamlines change direction, from being aligned along the drop surface to being aligned along the drop axis $^{4}$. Since solute has reached a certain number of streamlines, specifically those with streamfunction up to order $\sqrt{\zeta / P e}$, and since the streamlines are themselves beginning to penetrate very deeply into the drop interior, it follows that solute itself is penetrating deep into the drop interior along the axis (see also Figure 1(b)). Near the rear stagnation point then, the "boundary layer" containing the solute is no longer confined close to the drop surface making it challenging to keep proper account of the solute mass within it.

There is however an alternative way of computing solute mass $M$. This is to split the total solute mass that has entered the drop, into a part that is considered to be located near the drop surface and a part that is considered to have left the drop surface so as to be returned to the interior of the drop. To do this, we select arbitrarily a value $\theta_{\mathrm{r}}$ (with $\left.\pi-\theta_{\mathrm{r}} \ll 1\right)$, considered to be an angular location at which we deem mass effectively to have left the surface and to be returned to the interior. The near surface solute mass denoted $M_{\text {surf }}$ follows a formula identical to equation (14) but with integration limits 0 and $\theta_{\mathrm{r}}$ instead of 0 and $\pi$, hence

$$
M_{\text {surf }}=2 \pi \int_{0}^{\theta_{\mathrm{r}}} \sqrt{4 \zeta /(\operatorname{Pe} \pi)} \mathrm{d} \theta /\left.u_{\theta}\right|_{\text {surf }} .
$$

The solute mass entering or being returned to the interior denoted $M_{\text {ret }}$ meanwhile is obtained by integrating over time the total mass flux across $\theta_{\mathrm{r}}$

$$
M_{\mathrm{ret}}=\left.2 \pi \int_{0}^{T} \int_{0}^{n} w u_{\theta}\right|_{\operatorname{surf}, \theta=\theta_{\mathrm{r}}} \sin \theta_{\mathrm{r}}(1-n) \mathrm{d} T \mathrm{~d} n
$$

\footnotetext{
${ }^{4} \mathrm{An}$ analogous change in alignment direction of the streamlines also happens of course near the forward stagnation point, but is not found to be problematic (Ubal et al., 2010) for computing mass via equation (14) because $\sqrt{\zeta}$ tends to vanish more quickly than $\left.u_{\theta}\right|_{\text {surf }}$ does near the forward stagnation point at $\theta=0$. It is only the rear stagnation point $\theta=\pi$ that proves problematic for equation (14).
} 
As before, we can change variables from $n$ (distance from the drop surface) to streamfunction $\psi$, and shift the upper integration limit for $\psi$ to infinity. If we then substitute from equation (11) and perform the integral, we obtain

$$
M_{\text {ret }}=\left.2 \pi \int_{0}^{T} \int_{0}^{\infty} w\right|_{\theta=\theta_{\mathrm{r}}} \mathrm{d} T \mathrm{~d} \psi=2 \pi \int_{0}^{T} \sqrt{\left.4 \zeta\right|_{\theta=\theta_{\mathrm{r}}} /(P e \pi)} \mathrm{d} T .
$$

We emphasise that the split between near surface mass $M_{\text {surf }}$ and mass in the interior $M_{\text {ret }}$ is artificial, and is dependent on our choice of $\theta_{\mathrm{r}}$. By moving $\theta_{\mathrm{r}}$ closer to $\pi$ we increase $M_{\text {surf }}$ and decrease $M_{\text {ret }}$ by a compensating amount, so that the sum $M \equiv M_{\text {surf }}+M_{\text {ret }}$ should be unaffected. Nevertheless computing $M_{\text {surf }}$ and $M_{\text {ret }}$ as separate entities and summing them together, proves to be simpler than computing the integral (14) directly.

\subsubsection{Rate at which solute is crossing the drop surface}

Yet another way in which we can keep account of the total amount of mass within the drop is to keep track of the instantaneous rate at which solute is crossing the entire drop surface via diffusion, and integrating this rate over time.

If we denote this rate of change $\mathrm{d} M / \mathrm{d} T$ by $\dot{M}$, we deduce

$$
\dot{M}=\left.\left.\frac{2 \pi}{P e} \int_{0}^{\pi} \frac{\partial w}{\partial \psi}\right|_{\psi=0} \frac{\partial \psi}{\partial r}\right|_{r=1} \sin \theta \mathrm{d} \theta
$$

where the factor $1 / P e$ comes from the conversion between diffusive time $t$ and convective time $T$ (see equation (6)). By equation (11)

$$
\left.\frac{\partial w}{\partial \psi}\right|_{\psi=0}=-\frac{2}{\sqrt{\pi}} \sqrt{\frac{P e}{4 \zeta}}
$$

and by equation (5)

$$
\partial \psi /\left.\partial r\right|_{r=1}=-\left.u_{\theta}\right|_{\operatorname{surf}} \sin \theta
$$


giving finally ${ }^{5}$

$$
\dot{M}=\left.2 \pi \int_{0}^{\pi} \frac{1}{\sqrt{\pi P e \zeta}} u_{\theta}\right|_{\operatorname{surf}} \sin ^{2} \theta \mathrm{d} \theta
$$

Via this equation we can infer an interesting result. Most of the solute actually entering the drop tends to enter through those parts of the surface where $\zeta$ is smallest. Most of the solute actually accumulated within the drop is however located in those parts of the surface where $\zeta$ is largest (see equation (14)). The fact that there is a disconnect between the locations where solute enters the drop and the locations where solute actually accumulates indicates the effect of strong convection this high Peclet number system: it is relatively easy for convection to move solute around within the drop.

\subsection{Determining streamline orbit times}

We have said that the boundary layer solution (as presented in the previous section) should only persist up to the time corresponding to a single streamline orbit, and beyond that time a streamline-averaged theory should apply. We must therefore compute the streamline orbit times to determine when to switch from one theory to the other.

For a general streamline, if $s$ is variable measuring distance along that streamline, and $u_{s}$ is the fluid speed on the streamline (so that $u_{s}=\left(u_{r}^{2}+u_{\theta}^{2}\right)^{1 / 2}$ ) then the orbit time $T_{\text {orbit }}$ is straightforwardly defined as

$$
T_{\text {orbit }}=\oint \mathrm{d} s / u_{s}
$$

Here however we face a complication. We cannot deal with the orbit time of the streamline that passes exactly along the drop surface and drop axis, because that streamline contains stagnation points, and therefore has an infinite orbit time. Remembering that $\psi \equiv 0$ by definition on the drop surface and axis, it is possible to show that streamlines passing near the surface and/or axis have an orbit time $T_{\text {orbit }}$ satisfying

$$
T_{\text {orbit }} \sim A \log (1 / \psi)+B
$$

\footnotetext{
${ }^{5}$ Equivalence between solute mass predicted via this equation and via summing $M_{\text {surf }}$ and $M_{\text {ret }}$ follows by using equation (8) to eliminate $\left.u_{\theta}\right|_{\operatorname{surf}} \sin ^{2} \theta$ in favour of $\partial \zeta / \partial T$ and $\partial \zeta / \partial \theta$, and then integrating.
} 
where $A$ and $B$ are constants that can be readily obtained for any given flow fields $u_{r}$ and $u_{\theta}$ and where equation (23) applies for $\psi \ll 1$ (see Appendix A for details).

As time progresses, more and more mass enters the drop, and so reaches streamlines with higher and higher streamfunction, and hence (according to equation (23)) with lower and lower orbit times. Recall from section 2.2.2 that during the period when we deem the boundary layer solution to be valid, the solute concentration varies along as well as across streamlines. Indeed one could consider that, since there are still fluid elements on streamlines in the drop interior that have not yet been on the surface, those parts of the streamline effectively have $\zeta=0$ and hence (according to equation (11)) $w=0$. It is only at the end of the "boundary layer" phase of evolution that mass is considered to be reasonably uniformly distributed along streamlines.

This suggests the following procedure. At any given time $T$, we know the total amount of solute mass $M$ that has entered the drop. Some of this mass is distributed on streamlines arbitrarily close to the drop surface with arbitrarily long orbit times. Some of this mass is however distributed on streamlines which are slightly further from the surface with somewhat shorter orbit times. We can estimate a representative orbit time for any given amount of mass $M$ that has entered by considering that this same amount of mass is redistributed in a step function distribution with normalised mass fraction $w=1$ between $\psi=0$ (the drop surface and/or axis) and some value $\psi=\psi_{M}$, and with $w=0$ for $\psi>\psi_{M}$. Remembering how the mass fractions have been normalised via equation (3) we are effectively saying that for streamlines with $0<\psi<\psi_{M}$ the concentration field has equilibrated with the concentration outside the drop, whereas for $\psi>\psi_{M}$ the concentration is unchanged from the initial one. There is an unambiguous streamline orbit time $T_{\text {orbit }}\left(\psi_{M}\right)$ that is associated with the streamline $\psi_{M}$ : this provides an estimate of the representative orbit time when a given amount of solute mass $M$ has entered the drop, regardless of precisely how the mass is actually distributed across streamlines.

The implications of this definition of representative streamline orbit time are considered further in section 2.3.1, with section 2.3.2 then expanding the discussion to relate these orbit times back to volumes that the streamlines in question enclose. 


\subsubsection{Comparing streamline orbit times with time elapsed}

Clearly $\psi_{M}$ is an increasing function of $M$ : the more solute mass that has entered the drop, the more streamlines must be filled up with solute to store that mass. Note moreover that as time elapsed $T$ increases, $M$ increases, $\psi_{M}$ increases and hence $T_{\text {orbit }}\left(\psi_{M}\right)$ decreases. If $T_{\text {orbit }}\left(\psi_{M}\right)$ exceeds $T$ (as must happen for exceedingly small $T$ ) then it is clear that material on the streamline $\psi_{M}$ has not yet had sufficient time to execute a complete streamline orbit. Under those circumstances, the basis underlying the estimate referred to earlier (i.e. that solute mass can be redistributed uniformly both along and across streamlines between the drop surface/axis $\psi=0$ and a particular streamline $\psi=\psi_{M}$ ) is a poor approximation to the true way that the mass is distributed. As $T$ grows and $T_{\text {orbit }}\left(\psi_{M}\right)$ falls however, we eventually reach a point at which $T_{\text {orbit }}\left(\psi_{M}\right)=T$. Fluid has then had just enough time to orbit the streamline $\psi_{M}$, and we can expect therefore that mass is fairly uniformly distributed along that particular streamline. Our procedure then is, given the $M$ vs $T$ relation obtained via the boundary layer theory of section 2.2 , to find $\psi_{M}$ vs $T$, and hence the value of $T_{\text {orbit }}\left(\psi_{M}\right)$ such that $T_{\text {orbit }}\left(\psi_{M}\right)=T$. This then signals the time at which we should switch from a boundary layer theory to a streamline-averaged one. We denote this time by $T_{\text {switch }}$.

We reiterate that the above choice for the switching time $T_{\text {switch }}$ should be robust even though a step function distribution $w=1$ for $0<\psi<\psi_{M}$ and $w=0$ for $\psi>$ $\psi_{M}$ is artificial, and in reality some solute will have penetrated further than $\psi_{M}$ (albeit at a comparatively low concentration): $\psi_{M}$ is representative of the average distance in streamfunction space to which solute has penetrated, and hence $T_{\text {orbit }}\left(\psi_{M}\right)$ should likewise represent the average orbit time on those streamlines with significant solute concentration.

\subsubsection{Relating orbit times and volumes enclosed by streamlines}

Instead of treating the orbit time $T_{\text {orbit }}$ as a function of streamfunction $\psi$, we can also treat it as a function of enclosed volume (which we will denote $V$ ). Enclosed volume $V$ is defined here as the volume contained between the drop surface (streamfunction equal to zero) and an arbitrary streamline with streamfunction $\psi$. There is a simple relation 
between enclosed volume, orbit time and streamfunction (see Appendix B)

$$
V=2 \pi \int_{0}^{\psi} T_{\text {orbit }} \mathrm{d} \psi
$$

Given equation (23) applicable for streamlines near the drop surface and/or axis, the volume enclosed (again for near surface and/or near axis streamlines with $\psi \ll 1$ ) becomes

$$
V \sim 2 \pi A \log (1 / \psi) \psi+2 \pi(A+B) \psi
$$

Combining equations (23) and (24) gives a direct relation between $V$ and $T_{\text {orbit }}$

$$
\begin{aligned}
V & \sim 2 \pi\left(T_{\text {orbit }}-B\right) \exp \left(-\left(T_{\text {orbit }}-B\right) / A\right)+2 \pi(A+B) \exp \left(-\left(T_{\text {orbit }}-B\right) / A\right) \\
& =2 \pi\left(T_{\text {orbit }}+A\right) \exp \left(-\left(T_{\text {orbit }}-B\right) / A\right) .
\end{aligned}
$$

Notice that $V \rightarrow 0$ implies $T_{\text {orbit }} \rightarrow \infty$ which is as expected for streamlines passing arbitrarily close to the drop surface and axis.

The significance of the enclosed volume is as follows. If a mass $M$ of solute enters the drop and hypothetically were to be redistributed into a region with (normalised) solute fraction $w=1$, then the volume $V$ enclosed by that region would satisfy $V=M$. As time $T$ proceeds, the volume $V$ enclosing the solute filled region grows with time, but the orbit time $T_{\text {orbit }}$ for the particular streamline delineating the edge of that enclosed volume falls. Eventually a point is reached for which $T_{\text {orbit }}$ equals $T$ and that is the point at which we consider that a streamline-averaged formulation first becomes appropriate.

Note that according to section 2.2, the solute mass $M$ vs elapsed time $T$ relation (and hence the $V$ vs $T$ relation, since we are setting $V=M$ here) depends on Peclet number $P e$. This is evident from equation (15) and also from equation (17). The larger the Peclet number, the less mass that enters the drop in a given time $T$, and the less volume it fills. Since $V$ and $T_{\text {orbit }}$ are linked via equations (23) and (25), this means that systems with larger Peclet number require more units of time $T$ to satisfy the criterion $T_{\text {orbit }}=T$, and hence take longer to switch to a streamline-averaged state. Evidently the switching time 
$T_{\text {switch }}$ must increase as $P e$ increases.

This is however an artifact of $T$ having been made dimensionless on a convective time scale. If we convert to a time variable $t$ (made dimensionless on a diffusive time scale, and related to $T$ via equation (6)) the result is reversed: the larger the Peclet number, the more mass that enters the drop in a given time $t$, and the sooner the streamline-averaged state is achieved. The diffusive time scale is more relevant here, because in the streamlineaveraged state, mass transfer into the drop proceeds via cross-stream diffusion: details of the streamline-averaged theory are presented in the next section.

\subsection{Streamline-averaged theory}

The streamline-averaged theory was described by Ubal et al. (2010) but was not implemented in that work, although previous authors implemented it in the case of a very specific flow field (Brignell, 1975; Kronig and Brink, 1950; Prakash and Sirignano, 1978). The formulation can however be adapted to other flow fields as well, and the corresponding derivation of Ubal et al. (2010) is replicated here, including some additional insights over and above those offered by Ubal et al. (2010). The discussion that follows is divided into two parts: section 2.4.1 considers diffusion in generalised coordinate systems, whereas section 2.4.2 derives the streamline-averaged equation for solute mass transfer, the key result being equation (32) below. Readers already familiar with the derivation of this particular equation may prefer to skip directly to section 2.5.

\subsubsection{Convection-diffusion in a generalised coordinate system}

We start from the convection-diffusion equation presented in section 2.1 but change variables by defining a generalised coordinate system $s$ (distance along a streamline), $\psi$ (streamfunction) and $\phi$ (azimuthal angle), instead of the original $r, \theta, \phi$ spherical polars. Note that $s$ varies in the domain $0 \leq s \leq L_{\text {orbit }}(\psi)$ where $L_{\text {orbit }}$ is the length of a streamline orbit which depends on streamfunction $\psi$. We can however define a coordinate $S=s / L_{\text {orbit }}(\psi)$ and the domain for this is always $0 \leq S \leq 1$ independent of $\psi$. We can then employ coordinates $S, \psi$ and $\phi$.

A complication now arises in that, in general, the coordinates $S$ and $\psi$ are not orthog- 
onal to one another. Nevertheless, without loss of generality we can select one arbitrary streamline and define a coordinate $\mathcal{S}$ such that on that arbitrarily chosen streamline $\mathcal{S}=S$. Moving away from this particular streamline, we can construct curves of constant $\mathcal{S}$ by moving in the normal direction from streamline to streamline. Except in the special case when the streamlines are perfect concentric circles, as we move further and further from the originally chosen streamline, discrepancies between $\mathcal{S}$ and $S$ begin to develop. Nevertheless the domain of $\mathcal{S}$ remains the same as that of $S$, i.e. $0 \leq \mathcal{S} \leq 1$, and we also know that, along any given streamline, $\mathcal{S}$ is an increasing function of $S$ and vice versa.

By construction, $\mathcal{S}, \psi$ and $\phi$ form an orthogonal coordinate system and the streamlineaveraged theory is simplest to formulate for such a system. Scale factors for the $\mathcal{S}, \psi, \phi$ coordinate system are given in Appendix B. In particular the scale factor for $\mathcal{S}$ is $\chi L_{\text {orbit }}$ where $\chi \equiv(\partial S / \partial \mathcal{S})_{\psi}$ (and is a function of both $\mathcal{S}$ and $\left.\psi\right)$. For the particular streamline upon which we set $\mathcal{S}=S$, clearly $\chi \equiv 1$, but away from this streamline all we can say in general is that $\chi>0$ and $\int_{0}^{1} \chi \mathrm{d} \mathcal{S}=1$. Meanwhile the scale factor for $\psi$ is $\left(u_{s} r \sin \theta\right)^{-1}$ (see Appendix B) where $u_{s}$ is the fluid speed. The scale factor for $\phi$ is $r \sin \theta$.

With the above mentioned scale factors, equation (2) can be rewritten

$$
\frac{\partial w}{\partial t}+P e \frac{u_{s}}{\chi L_{\text {orbit }}} \frac{\partial w}{\partial \mathcal{S}}=\frac{u_{s}}{\chi L_{\text {orbit }}} \frac{\partial}{\partial \psi}\left(\chi L_{\text {orbit }} u_{s} r^{2} \sin ^{2} \theta \frac{\partial w}{\partial \psi}\right)+\frac{u_{s}}{\chi L_{\text {orbit }}} \frac{\partial}{\partial \mathcal{S}}\left(\frac{1}{u_{s} \chi L_{\text {orbit }}} \frac{\partial w}{\partial \mathcal{S}}\right)
$$

where the system is still taken to be axisymmetric, so has no dependence on $\phi$.

\subsubsection{Deriving the streamline-averaged equation}

The streamline-averaged theory proceeds by assuming that within equation (27) we can replace $w$ (which depends on $S, \psi$ and $t$ ) by a quantity $W$ given by

$$
W=\frac{1}{T_{\text {orbit }}} \oint \frac{w \mathrm{~d} s}{u_{s}}=\frac{1}{T_{\text {orbit }}} \oint \frac{w L_{\text {orbit }} \mathrm{d} S}{u_{s}}=\frac{1}{T_{\text {orbit }}} \oint \frac{w \chi L_{\text {orbit }} \mathrm{d} \mathcal{S}}{u_{s}}
$$

each element $\mathrm{d} s$ of the streamline, being weighted by the time $\mathrm{d} s / u_{s}$ to travel along it.

An evolution equation for $W$ is derived as follows. If we multiply equation (27) by 
$\chi L_{\text {orbit }} / u_{s}$, integrate over $\mathcal{S}$ and divide through by $T_{\text {orbit }}$ we obtain

$$
\frac{\partial W}{\partial t}=\frac{1}{T_{\text {orbit }}} \oint \mathrm{d} \mathcal{S} \frac{\partial}{\partial \psi}\left(\chi L_{\text {orbit }} u_{s} r^{2} \sin ^{2} \theta \frac{\partial w}{\partial \psi}\right)
$$

Assuming that $\partial w / \partial \psi$ on the right hand side of equation (29) can be replaced by $\partial W / \partial \psi$ (which is independent of $\mathcal{S}$ and so can be taken outside the integral), and swapping the order of integration with respect to $\mathcal{S}$ and differentiation with respect to $\psi$ leads to

$$
\frac{\partial W}{\partial t}=\frac{1}{T_{\text {orbit }}} \frac{\partial}{\partial \psi}\left(\left(\oint \chi L_{\text {orbit }} \mathrm{d} \mathcal{S} u_{s} r^{2} \sin ^{2} \theta\right) \frac{\partial W}{\partial \psi}\right)=\frac{1}{T_{\text {orbit }}} \frac{\partial}{\partial \psi}\left(\left(\oint \mathrm{d} s u_{s} r^{2} \sin ^{2} \theta\right) \frac{\partial W}{\partial \psi}\right) .
$$

We define an effective diffusivity via

$$
D_{\text {eff }}=\frac{1}{T_{\text {orbit }}} \oint \frac{\mathrm{d} s}{u_{s}} u_{s}^{2} r^{2} \sin ^{2} \theta
$$

This is the average along a streamline of the function $u_{s}^{2} r^{2} \sin ^{2} \theta$ weighted by the time $\mathrm{d} s / u_{s}$ taken to move along each streamline segment. Note that the expression $u_{s}^{2} r^{2} \sin \theta$ that we are averaging is analogous to the term on the right hand side of equation (8), except that equation (8) applied only on the drop surface whereas equation (30) applies to streamlines in the interior of the drop.

The physical interpretation is however the same. If the speed of the flow increases, streamlines move closer together and it is easier to exchange mass between them. Moreover (in the spherical geometry of the drop) as we move further from the drop axis, streamlines also move closer together, and again it becomes easier to exchange mass. Thus $D_{\text {eff }}$ really does permit an interpretation as a "diffusivity" albeit in a generalised streamfunction coordinate, not in physical space.

Substituting equation (31) into equation (30) leads to

$$
\frac{\partial W}{\partial t}=\frac{1}{T_{\text {orbit }}(\psi)} \frac{\partial}{\partial \psi}\left(D_{\text {eff }}(\psi) T_{\text {orbit }}(\psi) \frac{\partial W}{\partial \psi}\right)
$$

which is the same final equation as Ubal et al. (2010) obtained. 
Note that the $T_{\text {orbit }}$ term in the above equation cannot generally be cancelled out. It would only cancel from the equation in the special case of a uniform $T_{\text {orbit }}$ which implies a solid body rotation velocity field, which is not typically the case. In fact the $T_{\text {orbit }}$ term has a physical significance in terms of the volume enclosed between adjacent streamlines. The larger $T_{\text {orbit }}$, the larger the volume enclosed by adjacent streamlines (see e.g. equation (24)). For a given mismatch in the solute flux entering across one streamline and leaving across an adjacent one, the impact on the evolution of the mass fraction is less for a larger volume, and hence less for a larger $T_{\text {orbit }}$.

Equation (32) is an evolution equation for mass fraction, where details of the flow field, i.e. its kinematics and its geometry, are absorbed solely into the functions $D_{\text {eff }}(\psi)$ and $T_{\text {orbit }}(\psi)$, with the flow field having no other impact on the subsequent evolution.

Clearly computing these functions is of central importance, and they can be computed immediately once the streamline pattern is known. The converse is not however true: knowledge of the functions $D_{\text {eff }}(\psi)$ and $T_{\text {orbit }}(\psi)$ does not permit us to reconstruct the full streamline pattern, because $D_{\text {eff }}(\psi)$ and $T_{\text {orbit }}(\psi)$ provide only incomplete (i.e. integrated) information about the streamlines, and hence do not supply full details of how the streamlines are laid out in space. Equation (32) therefore utilizes incomplete information about the streamline field, but this still appears to be enough to estimate how solute is distributed in the drop over space and time. This equation must be solved subject to appropriate boundary and initial conditions which are discussed next.

\subsection{Boundary and initial conditions for streamline-averaged theory}

The boundary condition on the streamline at the drop surface and drop axis $\psi=0$ is $w=1$ (see section 2.1) and consequently $W=1$, i.e. solute at the drop surface is equilibrated with solute outside.

The boundary condition at the internal stagnation point is slightly more complicated to obtain. In physical space we know that $w$ should be a local minimum at that point, i.e. $\nabla w=0$ there or equivalently $\nabla W=0$. The problem however is that this does not necessarily imply that $\partial W / \partial \psi=0$ there. The reason is that $\nabla \psi=0$ at the stagnation point, and since $\nabla W=\partial W / \partial \psi \nabla \psi$, we can achieve $\nabla W=0$ at the stagnation point 
without $\partial W / \partial \psi$ needing to vanish. In fact it is possible to prove (see Appendix $\mathrm{C}$ ) that at the stagnation point, we have an evolution equation

$$
\partial W /\left.\partial t\right|_{\text {stag }}=-\partial W /\left.\partial \psi\right|_{\text {stag }} r_{\text {stag }} \sin \theta_{\text {stag }} \omega_{\text {stag }}
$$

where $\omega_{\text {stag }}$ is the vorticity at this point. Knowing the instantaneous value of $W$ at the stagnation point, and knowing how $W$ varies spatially between the stagnation point and a nearby streamline, equation (33) permits us to determine how $W$ at the stagnation point subsequently evolves over time.

Regarding initial conditions for the streamline-averaged formulation, the complication here is that the streamline-averaged formulation does not apply all the way down to zero time, but instead only commences at a finite time corresponding to one complete orbit of the near surface streamlines. It must therefore inherit its $W$ vs $\psi$ distribution from whatever mass fraction field preceded the onset of streamline averaging.

Indeed if we knew the exact distribution of the mass fraction $w$ with respect to both $\psi$ and $s$ at the critical time corresponding to the onset of streamline averaging, we could very simply compute the initial $W$ via equation (28). Were we to perform a full numerical simulation of the convection-diffusion equation, we would indeed know the mass fraction field $w$ at any given time, and could therefore employ equation (28) to obtain $W$.

Such full numerical simulations are however expensive, and we have already pointed out that it is desirable to use a boundary layer approach to describe the early-time behaviour. One of the issues with the boundary layer formulation, is that (as explained in section 2.2) we tend only to describe the mass fraction field near the drop surface up to an angular location $\theta=\theta_{\mathrm{r}}$ (with $\theta_{\mathrm{r}}<\pi$ but $\pi-\theta_{\mathrm{r}} \ll 1$ ). Beyond $\theta=\theta_{\mathrm{r}}$ we tend to say some mass has entered the interior of the drop, but we do not explicitly specify how it is distributed. This means that we cannot employ equation (28) which requires knowledge of the mass fraction field along entire streamlines. An alternative which does not require such knowledge is however discussed below. 


\subsubsection{Proposing an initial condition for $W$}

We note that at the time at which we switch from a boundary layer formulation to a streamline-averaged one, a known total amount of mass has entered the drop. We also note that the boundary layer mass fraction field as given by equation (11) strictly speaking does not have near uniform concentration on streamlines, not even on those parts of the streamline, i.e. near the drop surface, where the boundary layer theory manages to predict the concentration field. Instead the "extent of diffusion" term $\zeta$ in equation (11) is known to vary along the drop surface and hence along the streamwise direction, implying non-uniformities in $w$ along the streamwise direction also.

If we wish to replace equation (11) by a solute distribution which is uniform along streamlines, it makes sense to use a distribution $W$ (as an initial condition for the streamline-averaged model) with a broadly similar functional form. Hence we propose

$$
W=\operatorname{erfc}\left(\psi / \psi_{*}\right)
$$

where $\psi_{*}$ is a value that we must determine (and depends on Peclet number). We emphasise that this equation (34) is only considered to apply at the instant at which we switch to a streamline-averaged formulation, and that afterwards the field $W$ vs $\psi$ will evolve according to the governing equation (32).

As a bare minimum we require that, upon switching to a streamline-averaged formulation, the mass under the distribution $W$ in equation (34) matches the mass $M$ inherited from the boundary layer model (see section 2.2.3). To achieve this, we firstly need a general expression for the solute mass under the distribution $W$. Via equation (24) which relates volumes to $T_{\text {orbit }}$ and $\psi$, the solute mass $M$ can be obtained by integrating

$$
M=2 \pi \int_{0}^{\infty} W T_{\text {orbit }} \mathrm{d} \psi=2 \pi \int_{0}^{\infty} \operatorname{erfc}\left(\psi / \psi_{*}\right) T_{\text {orbit }} \mathrm{d} \psi
$$

The upper limit of the integration has been pushed to infinity here, reflecting the fact that we typically switch from a boundary layer formulation to a streamline-averaged one, at times such that the value of $M$ is still relatively small (ideally $M \ll 1$ ), meaning $\psi_{*}$ 
is likewise small, and hence much smaller than the full range of $\psi$ covering the entire drop: the term $\operatorname{erfc}\left(\psi / \psi_{*}\right)$ in the integrand is completely negligible whenever $\psi / \psi_{*} \gg 1$. At the opposite end of the integration domain, where $\psi \rightarrow 0$, we observe that $T_{\text {orbit }}$ (see equation (23)) in the integrand of equation (35) actually diverges, but the divergence is a weak logarithmic one, so the function remains integrable.

We employ equation (35) to define the $\psi_{*}$ value required to obtain a certain target mass $M$ inherited from the boundary layer model. Moreover since $M$ decreases as $P e$ increases (as is obvious from equation (14)), it follows that $\psi_{*}$ is also a decreasing function of Pe. Observe nevertheless that the rate of change of mass within the drop depends on the mass flux across the drop surface, and hence on the spatial gradient of mass fraction at the surface. By changing the assumed form of the mass fraction field adopting equation (34) in lieu of equation (11), we change the mass fraction gradients, thereby changing the rate of accumulation of mass within the drop. Hence, equation (34) recovers the correct amount of solute mass when we switch from a boundary layer formulation to a streamline-averaged formulation, but leads to a discontinuity in the rate of change of solute mass.

To an extent though, this discontinuity in the rate of change of mass is actually an effect we want to capture. Remember that the instant when we switch from the boundary layer theory to the streamline-averaged theory is supposed to represent the point when we switch from material being injected onto the drop surface from the drop axis never having been in contact with the drop surface, to material being injected onto the drop surface from the drop axis having previously been in contact with that surface. Thus it represents a transition whereby we see a sudden change from a very large spatial gradient in solute fraction across near surface streamlines to a much smaller spatial gradient across them, and thus a dramatic fall in the rate of accumulation of mass in the drop. Full numerical simulations do indeed show this effect (Ubal et al., 2010), albeit the abrupt decrease in rate of mass accumulation is not instantaneous, but rather is spread over a finite (but exceedingly small) time interval.

Note that an alternative way of distributing the solute mass $M$ at the instant when we switch to a streamline-averaged formulation would be to assume a step function solute 
distribution, i.e. $W=1$ for $\psi<\psi_{M}$ and $W=0$ for $\psi>\psi_{M}$ where $\psi_{M}$ is a parameter we defined in section 2.3. We already used this distribution in that section as a convenient way to estimate the appropriate time at which to switch to the streamline-averaged model. We do not however find this step function to be the most suitable to use as an initial condition for solving equation (34). On one hand, it has instantaneously zero mass fraction gradient at the drop surface, and hence instantaneously zero rate of mass accumulation but, on the other hand, the mass fraction gradient at the edge of the step $\left(\psi=\psi_{M}\right)$ is infinite at the same instant of time. Full numerical simulations (Ubal et al., 2010) show a sudden decrease in the rate of mass accumulation around one orbit time of a near surface streamline, but do not show the rate of mass accumulation being temporarily arrested altogether.

\subsection{Summary of theory/governing equations}

To summarize the discussion so far, we propose that during liquid-liquid extraction with a circulating drop, a boundary layer theory (see section 2.2) can describe the earlytime behaviour of the mass transfer into the drop up to a single streamline orbit time, but not any longer than that: Uribe-Ramirez and Korchinsky (2000b) extrapolated the boundary layer theory to times far longer than it actually applies, and hence overpredicted the mass transfer rate very significantly (Ubal et al., 2010). We identified (see section 2.3) the upper time limit for applicability of the boundary layer theory by determining how far into the drop the solute concentration field extends as a function of elapsed time $T$, and matching that time to the streamline orbit time $T_{\text {orbit }}$ (which itself falls as the solute concentration extends further and further): there is therefore a well-defined matching or switching point at which $T$ and $T_{\text {orbit }}$ become equal. This time is denoted $T_{\text {switch }}$ and it depends on Peclet number Pe. Beyond this time, a switch to the streamline-averaged theory is proposed (see sections 2.4-2.5), which involves diffusive mass transfer in the cross-stream direction. Despite the streamline-average theory being thereby diffusive in nature, it still retains some information about the streamline pattern, albeit incomplete information, involving just two parameters per streamline, the orbit time $T_{\text {orbit }}$ mentioned above and an effective diffusivity $D_{\text {eff }}$. The above then constitutes the mathematical model that we propose to describe mass transfer during liquid-liquid extraction. The 
methodology that we employ to implement the model is addressed in the next section.

\section{Solution methods}

Section 2 concerned itself with formulating the model used to describe mass transfer to a circulating drop during liquid-liquid extraction. The present section highlights the solution methodology that we use to implement the model. Since the theory of section 2 involves a number of diverse elements combined together into a single overall model, the solution methods we use likewise require us to bring together a number of diverse techniques. That said, each solution technique that we employ is actually a fairly standard one: as such, we have chosen to present the details of the numerical solution methods within an appendix (see Appendix D), using the present section merely to sketch out very briefly the main steps in the methodology that we follow, namely:

1. The fluid flow field is specified (with the aid of a Galerkin expansion).

2. The fluid flow field near the drop surface is used to determine (via numerical integrations) the structure of the solute concentration boundary layer, as well as the solute mass that enters the drop through the boundary layer.

3. The volume of the drop invaded by solute is examined versus the time elapsed, and a switching time is identified (via a Newton-Raphson technique) such that time elapsed matches the streamline orbit time associated with the volume invaded.

4. Following the switching time, a streamline-averaged approach is adopted; parameters to be input into the streamline-averaged model are determined by following the flow field along individual streamlines (again via numerical integrations).

5. Having computed the parameters to input into the streamline-averaged model, the model itself is solved (via finite differences).

Those readers requiring a fuller description of the techniques employed, along with the parameter values utilised in the model implementation, should consult Appendix D. 


\section{Results}

This section presents and discusses the predictions of the model for solute mass transfer in a circulating drop. The structure of this section mirrors the structure already used in section 2 to describe the model itself. In other words, first a boundary layer theory is considered and then this is subsequently switched to a streamline-averaged theory. Specifically in what follows section 4.1 examines data for $\theta_{0}(\theta, T)$ which is the initial location of a material point which currently finds itself at location $\theta$ at time T. Next section 4.2 examines results for the function $\zeta(\theta, T)$, the so called "extent of diffusion", in terms of which the boundary layer theory is expressed. After that section 4.3 examines solute masses transferred for the boundary layer theory (which can be obtained via the predictions for $\zeta)$ : together solute adjacent to the surface $M_{\text {surf }}(T)$ and solute that has been returned to the drop interior $M_{\text {ret }}(T)$ comprise the total solute mass $M(T)$. Section 4.4 then considers streamline orbit times $T_{\text {orbit }}(\psi)$, and also determines the time $T_{\text {switch }}$ at which the system switches from a boundary layer behaviour to a streamline-averaged one: as we have already explained, the criterion for finding this so called switching time is that the solute has invaded a sufficient volume of the drop that the fluid elements have managed to circulate exactly once around a streamline, so that all points on the streamline have passed through the same set of spatial locations, meaning that their solute concentrations should not be too dissimilar. Following this, the focus of the discussion changes towards the streamlineaveraged theory. Section 4.5 considers effective diffusivities $D_{\text {eff }}(\psi)$ which are essential input into the streamline-averaged theory. Section 4.6 considers the streamline-averaged results themselves, specifically data for streamline-averaged solute mass fractions $W(\psi, t)$, and also shows a global overview of the time evolution of the total amount of solute mass in the drop, spanning both the boundary layer and streamline-averaged behaviours.

\subsection{Results for angular location $\theta_{0}$}

Before determining the structure of the boundary layer it is necessary to determine the angular location $\theta_{0}$ which is a function of $\theta$ and $T$. Specifically $\theta_{0}$ given by equation (9) is the initial location of a fluid element that is currently at location $\theta$ at time $T$. 
Figure 2 plots $\theta_{0}$ vs $T$ for a selection of $\theta$ values. For any given $\theta$, it is clear that $\theta_{0}$ decreases as $T$ increases, reflecting the fact that material points must start off closer and closer to the forward stagnation point of the drop if they are to reach location $\theta$ by time $T$. Moreover Figure 2 shows that the larger the value of $\theta$, the longer it takes for $\theta_{0}$ to decrease down to small values (i.e. $\theta_{0}$ much smaller than unity).

According to equation (9) it would take an arbitrarily long time for $\theta_{0}$ to fall to arbitrarily small values. In reality however it is unnecessary to consider arbitrarily small $\theta_{0}$. The value of the integral (10) determining the extent of diffusion function $\zeta$ is extremely insensitive to the choice of $\theta_{0}$ provided $\theta_{0} \ll 1$. As is explained more fully in Appendix D.2, it is possible to define an injection point, denoted $\theta_{\mathrm{i}}$, at which fluid elements are considered to be injected from the interior of the drop onto the drop surface. Equation (10) is then modified to equation (D.4) which turns out to make it unnecessary to specify $\theta_{0}$ values less than $\theta_{\mathrm{i}}$. Moreover any choice of $\theta_{\mathrm{i}} \ll 1$ gives effectively the same predictions of $\zeta$ : as Appendix D.2.3 explains, equation (D.4) is (like equation (10) itself) very insensitive to its lower integration bound, as long as that lower bound is small.

Returning to consider the data in Figure 2, the dash-dot line at the bottom of the figure shows a selected value of $\theta_{\mathrm{i}}$ (chosen as $\theta_{\mathrm{i}}=\frac{\pi}{100}$ here). For the flow field assumed here (see Appendix D.1) and for the largest $\theta$ value considered (namely $\theta=\theta_{\mathrm{r}}$ chosen to be $\theta_{\mathrm{r}}=\frac{99 \pi}{100}$ in Appendix D.2), it takes a time $T \approx 18.08$ for $\theta_{0}$ to fall to the value $\theta_{\mathrm{i}}$. Beyond that time, for any $\theta<\theta_{\mathrm{r}}$, the corresponding $\theta_{0}$ value becomes less than $\theta_{\mathrm{i}}$.

This completes our discussion of the function $\theta_{0}$. Our primary interest in this function is not so much for its own sake, but rather because it enables us to compute the so called extent of diffusion $\zeta$ (via equation (10) or equation (D.4)), with $\zeta$ being needed in turn to compute the amount of solute mass in the drop. Results for the behaviour of $\zeta$ are considered in the next subsection.

\subsection{Results for extent of diffusion $\zeta$}

The extent of diffusion function $\zeta$ (which depends on both $\theta$ and $T$ ) is a measure of how much solute diffusion has taken place across a boundary layer at the drop surface. As has been described in section $2.2, \zeta$ behaves analogously to a "diffusive clock" which 
runs fast when near-surface streamlines are close together (rapid diffusion from streamline to streamline) and which runs slow when near-surface streamlines spread far apart (slow diffusion from streamline to streamline). As is evident from the solute concentration field in equation (11), solute is confined to those streamlines that satisfy $\psi \leq O(\sqrt{\zeta / P e})$ where $P e$ is the Peclet number. Knowing $\zeta$ thereby permits us to know the amount of solute within the drop, and how far that solute has penetrated.

In what follows we will consider plots of $\zeta$ vs $\theta$ for a selection of different times $T$ (always assuming that time $T$ is smaller than $T_{\text {switch }}$ so that a boundary layer theory, rather than a streamline-averaged theory, applies). Specifically we will consider values $T=1, T=5$ and $T=10$. Given that later on (see section 4.4 ) we will show that $T_{\text {switch }}$ tends to be a little larger than 10, these chosen values for $T$ are actually sensible. We will find (see sections 4.2.1-4.2.2) that there exists a very early-time limit in which the solute mass transfer into the drop is actually independent of convection even though the definition of $\zeta$ inherently involves convection. Likewise we will find (in sections 4.2.34.2.6) that there exists a later time limit in which $\zeta$ evolves into a steady state that depends on $\theta$, but not on $T$. One key result we will demonstrate (already anticipated in section 4.1) is that $\zeta$ is very insensitive to the choice of the injection angle $\theta_{\mathrm{i}}$

\subsection{1. $\zeta$ vs $\theta$ at time $T=1$}

Figure 3(a) plots $\zeta$ vs $\theta$ for $T=1$. It is evident that at this particular time, $\zeta$ has a peak close to the equator of the drop, but its value becomes much smaller approaching either the forward or rear stagnation point of the drop. This is the general sort of behaviour that we expect to see in the small time limit. When $T$ is small, such that a material point has migrated only very little from its initial position (or equivalently such that $\theta$ and $\theta_{0}$ are close together), we approximate equation (10) or equation (D.4) by

$$
\left.\zeta \approx\left(\theta-\theta_{0}\right) u_{\theta}\right|_{\text {surf }} \sin ^{2} \theta
$$


Since in addition equation $(9)$ becomes $\theta-\left.\theta_{0} \approx u_{\theta}\right|_{\text {surf }} T$ in the limit of "very early" times 6 , an approximate formula follows

$$
\left.\zeta \approx T u_{\theta}^{2}\right|_{\operatorname{surf}} \sin ^{2} \theta
$$

This approximate formula is also plotted in Figure 3(a), setting as before $T=1$.

Whilst this shows the same general behaviour as the true $\zeta$ vs $\theta$ function, by the time $T=1$ there is nonetheless an already noticeable difference between the true $\zeta$ and the approximate one. The peak value of the approximate $\zeta$ is higher than that of the true $\zeta$ and it is also located further upstream. In fact the peak value of equation (37) is located at the point where $\left.u_{\theta}\right|_{\operatorname{surf}} \sin \theta$ is maximal, and given the specific functional form chosen for $\left.u_{\theta}\right|_{\text {surf }}$ (see Appendix D.1.1) this occurs slightly upstream of the equator.

In other words, the peak value of the true $\zeta$ is shifted slightly downstream relative to the peak value of $\left.u_{\theta}\right|_{\operatorname{surf}} \sin \theta$, which is unsurprising since equation (8) indicates that values of $\zeta$ are convected along. This also explains why the peak of the true $\zeta$ is less high than the peak of equation (37). Following a material element, the highest rate of growth of $\zeta$ occurs where $\left.u_{\theta}\right|_{\operatorname{surf}} \sin \theta$ is largest, but no single material element ever feels that highest rate of growth for the entire time interval $0 \leq T \leq 1$.

If we move some distance upstream from the equator, the true $\zeta$ tends to be less than the approximate one, because historically $\zeta$ (following a material element) had rates of growth upstream less than the instantaneous growth rate at the element's current location. On the other hand, if we move downstream from the equator, the true $\zeta$ exceeds the approximate one: historically $\zeta$ exhibited faster growth rates than the instantaneous rate at an element's current location.

\subsubsection{Spherically symmetric concentration field}

Equation (37) has a very simple physical interpretation. If we substitute equation (37) into equation (11) and recognize near the surface of the drop that $\partial \psi / \partial r \approx-\left.u_{\theta}\right|_{\operatorname{surf}} \sin \theta$,

\footnotetext{
${ }^{6}$ We refer to these times as "very early" as a reminder that we are considering the early stages of the evolution of the boundary layer, with the boundary layer model itself being an early-time theory compared to the streamline-averaged model.
} 
then we deduce a solute concentration field in the drop

$$
w \approx \operatorname{erfc}((1-r) \sqrt{P e} / \sqrt{4 T})
$$

Converting from convective time $T$ to diffusive time $t$ (with $t=T / P e$ ) implies

$$
w \approx \operatorname{erfc}((1-r) / \sqrt{4 t})
$$

This then is a spherically symmetric solute concentration field which (provided $t \ll 1$ ) corresponds to the similarity solution arising for a so called rigid drop: mass transfer is purely diffusive without any contribution from convection.

That such a "rigid drop" solution should apply at very early times is unsurprising (and has already been established by Ubal et al. (2010)). Solute concentration gradients are initially arbitrarily sharp, meaning that initially diffusion always dominates convection no matter how large the Peclet number $P e$ is. The value of $P e$ governs not whether diffusion initially dominates convection, but rather the length of time for which that situation is allowed to persist. In summary then, for convective time $T=1$ (or equivalently for diffusive time $t=1 / P e)$, the $\zeta$ profile that we obtain implies a solute concentration field in the drop that is still qualitatively similar to the spherically symmetric concentration field that would arise solely from diffusion, ignoring any effects of convection. This situation cannot however continue indefinitely as time $T$ increases, as we see in the next section.

\subsection{3. $\zeta$ vs $\theta$ at time $T=5$}

A plot of $\zeta$ vs $\theta$ at time $T=5$ is given in Figure 3(b). As before, the value of $\zeta$ remains small at the forward and rear stagnation points of the drop, reaching a peak value at an intermediate $\theta$ value. By contrast with Figure 3(a) however, we see that the peak value is now shifted well downstream of the equator of the drop: this is the effect of convection.

Yet another observation is that the height of the peak in Figure 3(b) is about twice that in Figure 3(a) although the time $T$ is 5 times higher. This is a strong indication that the concentration field, has departed strongly from the spherically symmetric "rigid drop" case (because otherwise equation (37) would predict $\zeta$ being proportional to $T$ ). 
This is corroborated by the study of Ubal et al. (2010) which presents data for full numerical simulations of the advection-diffusion equation. In that study, concentration fields (albeit fields for $P e=1000$ rather than $P e=10000$ ) are plotted at various times, and whereas the data for $T=2$ show only a modest departure from spherical symmetry, by time $T=5$ the deviation from spherical symmetry is already very strong.

Figure 3(b) makes a distinction between material points which have been continuously on the drop surface since time zero (those for which the original location $\theta_{0}$ exceeds the injection point $\theta_{\mathrm{i}}$ ) and material points which have been newly injected from the interior of the drop (those for which $\theta_{\mathrm{i}}$ exceeds $\theta_{0}$ implying that the material point only entered the surface at location $\theta_{\mathrm{i}}$ for time $T>0$ ). It is supposed here that material points are injected at the location $\theta_{\mathrm{i}}=\frac{\pi}{100}$. With this choice of $\theta_{\mathrm{i}}$ and at time $T=5$, roughly speaking half of the surface (the rear half) consists of points continuously on the surface since time zero, whilst the other half (the forward half) consists of newly injected points. However the balance between points continuously on the surface and newly injected ones changes according to the value of $\theta_{\mathrm{i}}$. Verification that such changes have nonetheless only very limited effect upon the value of $\zeta$ is provided in the next subsection.

\subsubsection{Effect of varying $\theta_{\mathrm{i}}$ upon $\zeta$ at $T=5$}

According to equation (D.4) the value of $\zeta$ can depend upon the choice for $\theta_{\mathrm{i}}$ although only points on the forward part of the drop (i.e. only points with $\theta_{0}<\theta_{\mathrm{i}}$ ) are affected. However it is demonstrated in Appendix D.2.1 that despite $\zeta$ being in principle sensitive to $\theta_{\mathrm{i}}$, that sensitivity is actually very weak: if $\theta_{\mathrm{i}}$ changes, then the maximum change in $\zeta$ at any given $\theta$ value is on the order of $\theta_{\mathrm{i}}^{4}$.

To quantify the effect of changing $\theta_{\mathrm{i}}$, in Figure $4(\mathrm{a})$ we examine (still for time $T=5$ ) a zoomed view of $\zeta$ vs $\theta$ for two different $\theta_{\mathrm{i}}$ values $\left(\frac{\pi}{100}\right.$ and $\left.\frac{\pi}{10}\right)$ focussing on the forward part of the drop, $\theta<\frac{\pi}{4}$, which is where changes in $\zeta$ with changing $\theta_{\mathrm{i}}$ are most apparent. The differences we see between the $\zeta$ values in these two different cases within Figure 4(a) are really very small, with the $\zeta$ values for $\theta_{\mathrm{i}}=\frac{\pi}{100}$ being slightly larger than those for $\theta_{\mathrm{i}}=\frac{\pi}{10}$. A further reduction in the $\theta_{\mathrm{i}}$ value, down to $\theta_{\mathrm{i}}=\frac{\pi}{1000}$ say, would lead to a further increase in $\zeta$ but this would be 4 orders of magnitude smaller than the difference already 
seen in Figure 4(a). In what follows therefore we select $\theta_{\mathrm{i}}=\frac{\pi}{100}$ confident that this choice has no significant impact on the solute mass transfer that we compute as a result.

\subsubsection{Comparing $\zeta$ at $T=5$ with steady state $\zeta_{\mathrm{ss}}$}

We have just verified that $\zeta$ is relatively insensitive to $\theta_{\mathrm{i}}$, the effect of $\theta_{\mathrm{i}}$ only manifesting itself via very weak sensitivity of $\zeta$ to the lower integration bound of equation (D.4). Interestingly we can draw a similar conclusion regarding $\zeta$ being relatively insensitive to time: this is because the time dependence within equation (D.4) only manifests itself via the term $\theta_{0}$ which appears in that same lower integration bound. It can be shown (see Appendix D.2.2-Appendix D.2.3) that, as time $T$ increases, $\zeta$ vs $\theta$ evolves towards a steady state profile that we denote $\zeta_{\mathrm{ss}}$. If, by $T=5$, the value of $\zeta$ has become relatively insensitive to time as we now anticipate, $\zeta$ should already be relatively close to $\zeta_{\mathrm{ss}}$.

In Figure 4(b) we compare $\zeta$ vs $\theta$ for $T=5$ with the steady state profile $\zeta_{\text {ss }}$. We see that $\zeta$ is actually very close to $\zeta_{\mathrm{ss}}$ over most of the surface of the drop (the neighbourhood of the rear stagnation point being an exception, i.e. convergence of $\zeta$ to $\zeta_{\mathrm{ss}}$ is not uniform).

In the forward half of the drop, agreement between $\zeta$ and $\zeta_{\mathrm{ss}}$ is unsurprising. Restricting consideration to points that have been newly injected onto the drop surface (i.e. those with $\theta_{0}<\theta_{\mathrm{i}}$ ), the lower integration bound in equation (D.4) is no longer time dependent, so steady state is certainly attained. The more significant finding is in the rear half of the drop, up to and including the $\theta$ value corresponding to the peak value of $\zeta$. For $T=5$, all the $\zeta$ values on this part of the drop whilst not exactly at steady state, do have $\theta_{0}$ values which, despite being greater than $\theta_{\mathrm{i}}$, are nevertheless much smaller than unity. This means that $\zeta$ evolves very slowly as time evolves: all that happens is that $\theta_{0}$ gradually decreases towards $\theta_{\mathrm{i}}$, but equation (D.4) is barely affected. We analyse how this gradual evolution proceeds by considering an even later time within the next section.

\subsection{6. $\zeta$ vs $\theta$ at time $T=10$}

In Figure 3(c) we show the $\zeta$ vs $\theta$ profile for $T=10$. Qualitatively this is similar to the $T=5$ profile already seen in Figure 3(b), namely $\zeta$ is small both at the forward and rear stagnation points of the drop, and there is a peak value of $\zeta$ at a certain $\theta$ value (which 
owing to convection is pushed far downstream of the drop equator). The differences seen at $T=10$ compared to $T=5$, are that for the longer time, the peak is shifted even further downstream, and a result there is a very sharp decrease in $\zeta$ from the peak value down to the rear stagnation point.

As was also done in Figure 3(b), a distinction is made in Figure 3(c) between points that have been continuously on the drop surface and points newly injected onto the surface since time zero (still assuming $\theta_{\mathrm{i}}=\frac{\pi}{100}$ ). Whereas in Figure 3(b) the drop surface was roughly equally split between the two types of points, here in Figure 3(c) it is the newly injected points that cover almost all the surface of the drop.

As discussed in section 4.2.3, the $\zeta$ values at these "newly injected" locations are already at steady state. Only in the relatively small region to the right of the peak $\zeta$ value, i.e. in the region of the sharp drop, is any time dependence of $\zeta$ still manifest.

As mentioned earlier, we are typically only interested in solving for $\zeta$ up as far as a "return point" $\theta_{\mathrm{r}}$ (at which point solute is considered to be simply returned to the interior of the drop). Once the $\theta_{0}$ value corresponding to $\theta=\theta_{\mathrm{r}}$ has fallen to the value $\theta_{\mathrm{i}}$, it is the case that $\zeta$ has attained $\zeta_{\text {ss }}$ globally throughout the domain $\theta_{\mathrm{i}} \leq \theta \leq \theta_{\mathrm{r}}$. Assuming that $\theta_{\mathrm{i}}=\frac{\pi}{100}$ and $\theta_{\mathrm{r}}=\frac{99 \pi}{100}$ this has not yet happened by time $T=10$ (as was mentioned in section 4.1, it does not happen until $T=18.08)$. Nevertheless it is clear from Figure 3(c) that by time $T=10$ the fraction of the drop surface over which $\zeta$ is still varying with time is really very small indeed.

This completes our discussion of the "extent of diffusion" $\zeta$ as a function of $\theta$ and $T$. The key finding is that there is an early-time behaviour ( $\zeta$ proportional to $T$ ) corresponding to a "rigid drop" (i.e. a spherically symmetric, purely diffusive mass transfer), but as time progresses the growth in $\zeta$ is arrested and a steady state $\zeta_{\mathrm{ss}}$ is attained. The implications for mass transfer into the drop are considered in the next section.

\subsection{Results for solute mass transferred according to boundary layer theory}

This section considers the solute mass transfer into the drop as predicted by the boundary layer theory. This can be obtained in terms of the extent of diffusion $\zeta$ using equation (15) (or more correctly by a slightly modified version thereof, equation (D.8) 
given in Appendix D.2.6), that gives the solute mass adjacent to the surface of the drop $M_{\text {surf }}$, and equation (17), which gives the solute mass $M_{\text {ret }}$ which passed through boundary layer but which has been now returned the interior of the drop. The total solute mass that has entered the $\operatorname{drop} M$ is the sum of $M_{\text {surf }}$ and $M_{\text {ret }}$.

Although we have performed a detailed analysis of how $M_{\text {surf }}, M_{\text {ret }}$ and $M$ evolve with time, the end result that we find is a rather surprising one. To a very good approximation, the amount of solute in the drop predicted by the boundary layer theory can be determined by identifying an abrupt transition between a very early-time state (in which mass transfer is dominated by a purely diffusive boundary layer with no effect whatsoever of convection) and a later time state (in which a convective boundary layer has fully developed into a steady state structure, albeit a streamline-averaged state is not yet attained). It turns out therefore that using the boundary layer theory to compute how solute mass transfer rates evolve at intermediate times is not strictly necessary: it is sufficient to know just the very early-time asymptotic behaviour of $\zeta$ and its later time asymptotic behaviour. In spite of this, computing the full time evolution of $\zeta$ (as we did in section 4.2) has been an essential step in the process, because it is only by doing so that we demonstrate the dominance of the aforementioned early- and late-time behaviours.

The rest of this section is laid out as follows. Section 4.3.1 considers the near surface solute mass $M_{\text {surf }}$, section 4.3.2 considers the solute mass returned to the interior of the $\operatorname{drop} M_{\text {ret }}$ and section 4.3 .3 considers the total mass $M \equiv M_{\text {surf }}+M_{\text {ret }}$. Finally section 4.3.4 presents a simple (but remarkably accurate) expression predicting $M$ as a function of $T$. Only the simple expression for $M$ vs $T$ from section 4.3 .4 needs to be carried forward to later results (section 4.4 onwards). Nonetheless the results from sections 4.3.1-4.3.3 explain how and why that simple expression can come about.

As long as the Peclet number $P e$ is sufficiently large that a boundary layer theory actually applies, the thickness of the boundary layer (see e.g. equations (17) and (D.8)) is proportional to $P e^{-1 / 2}$. Hence by multiplying solute masses by $P e^{1 / 2}$ we can collapse together data for all different Peclet numbers (assuming Peclet number is large): the data discussed here are therefore mostly expressed terms of $P e^{1 / 2} M_{\text {surf }}, P e^{1 / 2} M_{\text {ret }}$ and $P e^{1 / 2} M$. 


\subsubsection{Near surface mass in the boundary layer}

Figure 5(a) plots the computed $P e^{1 / 2} M_{\text {surf }}$ vs $T$. We see that $M_{\text {surf }}$ grows initially very rapidly, but then the rate of growth slows, and $M_{\text {surf }}$ eventually attains a final steady value.

The very rapid initial growth corresponds to the initial period during which mass transfer is controlled entirely by diffusion. We know that solute mass fraction $w$ obeys equation (39) and integrating this over $r$ (in the limit of very small $t$ ) and multiplying by the surface area of the drop (namely $4 \pi$ ) gives the solute mass (Ubal et al., 2010). Hence

$$
M_{\text {surf }} \approx 8 \sqrt{\pi t}
$$

and moreover (since $t=T / P e)$

$$
P e^{1 / 2} M_{\text {surf }} \approx 8 \sqrt{\pi T}
$$

This is also plotted in Figure 5(a). It is seen that this early-time formula starts off agreeing very well with the true $M_{\text {surf }}$ predicted by equation (D.8), although as time proceeds the early-time formula starts to fall beneath the true $M_{\text {surf }}$.

This completes, for the present, discussion of the mass adjacent to the drop surface $M_{\text {surf }}$. There is however another component contributing to the solute mass $M$, i.e. the mass that has been returned to the drop interior $M_{\text {ret }}$. This is discussed in what follows.

\subsubsection{Mass returned to the interior of the drop}

Figure 5(b) plots $P e^{1 / 2} M_{\text {ret }}$ vs $T$ as computed via equation (17). As is clear from equation (17), as long as $\zeta$ evaluated at $\theta=\theta_{\mathrm{r}}$ is negligible, then $M_{\text {ret }}$ is also negligible: in fact we see from Figure 5(b) that $M_{\text {ret }}$ is negligible for the first 10 time units or so.

Thereafter $M_{\text {ret }}$ then begins to increase, quickly attaining a nearly steady state rate of increase. Although a true steady state rate of increase is not achieved in principle until 18.08 time units, a near steady rate of increase is achieved somewhat before then: in order for this to occur, it is sufficient to have $\left.\theta_{0}\right|_{\theta=\theta_{\mathrm{r}}}$ being significantly smaller than unity (see also Figure 2 remembering that $\theta_{\mathrm{r}}=\frac{99 \pi}{100}$ here). Once that happens $\left.\zeta\right|_{\theta=\theta_{\mathrm{r}}}$ varies only very little with time, and this determines how $M_{\text {ret }}$ grows via equation (17). 
As mentioned above, once $M_{\text {ret }}$ begins to grow, it quickly attains a steady growth rate. We denote this by $\dot{M}_{\text {ret,ss }}$ and it can be readily estimated as is now explained.

For $M_{\text {ret }}$ to be steadily increasing, it follows via equation (17) that $\left.\zeta\right|_{\theta=\theta_{\mathrm{r}}}$ must be close to the corresponding steady state value $\left.\zeta_{\mathrm{ss}}\right|_{\theta=\theta_{\mathrm{r}}}$. At steady state, the lower integration bound of equation (D.4) is invariably $\theta_{\mathrm{i}}$ not $\theta_{0}$. To approximate $\left.\zeta_{\mathrm{ss}}\right|_{\theta=\theta_{\mathrm{r}}}$ we replace the lower integration bound $\theta_{\mathrm{i}}$ by zero, and the upper integration bound $\theta_{\mathrm{r}}$ by $\pi$, and thus obtain a quantity that we denote $\left.\zeta_{\mathrm{ss}}\right|_{\theta=\pi}$ via

$$
\left.\zeta_{\mathrm{ss}}\right|_{\theta=\pi}=\left.\int_{0}^{\pi} u_{\theta}\right|_{\mathrm{surf}} \sin ^{2} \theta \mathrm{d} \theta
$$

In our system $\left.u_{\theta}\right|_{\text {surf }}$ is described by equation (D.2) with parameters $\alpha$ and $\beta$ quoted in Appendix D.1.1, and we deduce that

$$
\left.\zeta_{\mathrm{ss}}\right|_{\theta=\pi}=\frac{4}{3} \alpha
$$

Notice that $\left.\zeta_{\mathrm{ss}}\right|_{\theta=\pi}$ is affected only by $\alpha$ (since the term involving $\beta$ cancels).

Employing $\left.\zeta_{\mathrm{ss}}\right|_{\theta=\pi}$ in lieu of $\left.\zeta\right|_{\theta=\theta_{\mathrm{r}}}$ within equation (17), the steady state value attained for the growth rate of $M_{\text {ret }}$, which we denote $\dot{M}_{\text {ret,ss }}$, becomes

$$
\dot{M}_{\text {ret,ss }}=2 \pi \sqrt{\frac{4}{P e \pi} \frac{4 \alpha}{3}} .
$$

Note that for the $\alpha$ value $(\alpha=0.590)$ considered here we find $\left.\zeta_{\text {ss }}\right|_{\theta=\pi} \equiv 4 \alpha / 3 \approx 0.786$. Coincidentally this is remarkably close to $\frac{\pi}{4} \approx 0.785$. To a very good approximation then

$$
P e^{1 / 2} \dot{M}_{\mathrm{ret}, \mathrm{ss}} \approx 2 \pi
$$

This formula is not of course general: it only works for the flow field with the particular $\alpha$ value considered here. Nonetheless it will prove useful in the analysis to follow.

This completes our discussion of $M_{\text {ret }}$, the solute mass that has been returned to the drop interior. In the next section we combine this with the solute mass adjacent to the surface $M_{\text {surf }}$ in order to determine the total solute mass $M$ in the drop. 


\subsubsection{Combined solute mass in the drop}

Figure 6(a) plots $P e^{1 / 2} M$ vs $T$ along with its constituent components $P e^{1 / 2} M_{\text {surf }}$ and $P e^{1 / 2} M_{\text {ret }}$. Here we make an interesting observation. Not only does $M_{\text {ret }}$ start growing around the same time that growth in $M_{\text {surf }}$ stops, in addition to this, the growth rates of $M_{\text {ret }}$ after this time and $M_{\text {surf }}$ before this time are matched. The net effect is that $M$ has a very substantial period of time during which it grows at a nearly constant rate, and such growth happens from really quite early on in the time evolution, certainly well before the time $T=18.08$ at which a true steady state is achieved.

That the near steady rate of growth of $M_{\text {surf }}$ prior to $M_{\text {surf }}$ saturating at a final steady value must match the near steady rate of growth of $M_{\text {ret }}$ after $M_{\text {ret }}$ has begun to grow, can also be explained as follows. The sum of the rates of change of $M_{\text {surf }}$ and $M_{\text {ret }}$ must equate to the rate at which solute is diffusing across the drop surface, an expression for which ${ }^{7}$ has been given in equation (21) within section 2.2.4.

The rate at which solute is diffusing across the drop surface tends to be dominated by those parts of the drop in which $\zeta$ remains relatively small (i.e. those parts of the drop on which the near surface layer containing solute is comparatively thin). The plots in e.g. Figure 3(b) and 3(c), show that these correspond to the upstream part of the drop. Moreover as is clear by comparing Figure 3(b) with 3(c), these upstream parts of the drop are also those in which $\zeta$ reaches steady state first. Thus the sum of the rates of change of $M_{\text {surf }}$ and $M_{\text {ret }}$ must reach steady state, even though the individual rates of change of $M_{\text {surf }}$ and $M_{\text {ret }}$ are not yet at steady state.

The value of $\dot{M}$ initially contributes almost exclusively to the growth of $M_{\text {surf }}$, with $M_{\text {ret }}$ remaining insignificant (see Figure 6(a)). Over time moreover, the distribution of solute mass contributing to $M_{\text {surf }}$ starts to shift, with the larger $\zeta$ values (which also make the larger contributions to $M_{\text {surf }}$ according to equation (D.8)) managing to migrate further and further downstream. Eventually the solute "breaks through" the location $\theta=\theta_{\mathrm{r}}$, after which $M_{\text {surf }}$ stops changing, whereas $M_{\text {ret }}$ starts to grow (again see Figure 6(a)). The exact

\footnotetext{
${ }^{7}$ We will not present any numerical data computed via equation (21) here since there are technical difficulties in evaluating equation (21) when describing $\zeta$ via equation (D.4) in place of equation (10) as we do here. Although these difficulties can be overcome, they have no bearing on the discussion to follow.
} 
time at which this breakthrough occurs is sensitive to the (arbitrary) choice of $\theta_{\mathrm{r}}$ but the value of $\dot{M}$ (both before and after breakthrough) does not depend on $\theta_{\mathrm{r}}$, having been already set by processes occurring upstream long before breakthrough occurs.

The above analysis also resolves a paradox which would otherwise arise when comparing these predictions that include solute convection to rigid drop predictions without any convection. We know from Figure 5(a) that the mass accumulated in the drop with convection exceeds the rigid drop predictions, implying that (at least part of) the near surface layer must contain more solute mass, i.e. be thicker, than the analogous situation for a rigid drop. Normally however the thicker a layer is, the slower mass diffuses into it. Hence the convective case cannot have a near surface layer that is uniformly thicker than the rigid drop case over the entire drop surface. There must be a region somewhere for which this near surface layer containing solute remains thin, permitting large concentration gradients and rapid mass transfer there. Figure 7 corroborates that this is indeed the case. The boundary layer is kept thin at the forward end of the drop as long as fresh material (which has not yet been in contact with solute) is brought from the drop interior to the surface: this maintains the sharp concentration gradients.

In the section that follows, we use what we know about the solute mass transport properties of this boundary layer at very early- and at later times to predict the evolution of the total solute mass in the drop over the entire range of times applicable to the boundary layer model.

\subsubsection{Combining early-time and later time growth for $M$}

In section 4.3.1 we found that the very early-time growth of the solute mass $M$ was controlled by diffusive "rigid drop" boundary layer behaviour (with $M$ proportional to $\sqrt{T}$ ). Then in section 4.3.2 we found that the later time growth of $M$ (albeit still within the boundary layer regime) corresponded to a steady rate of increase, but (for reasons that were explained) the system locks into a nearly steady state surprisingly soon, certainly much sooner than was originally expected.

Figure 6(b) shows a plot of $M$ vs $T$ as predicted by the boundary layer theory, but with the very early-time and late-time asymptotic behaviours from that theory superposed 
upon it. What we notice is that the transition between the early-time and late-time asymptotic behaviour is very abrupt. There is not any significant period of time when the system matches neither one nor the other asymptotic behaviour.

This suggests that to a good approximation, we can assume that the transition between the early-time and late-time states is instantaneous. We determine the time at which the transition occurs by requiring continuity of $\dot{M}$ through the transition. Equations (41) and (45) then require that the transition time ${ }^{8} T_{\text {trans }}$ is $T_{\text {trans }} \approx \frac{4}{\pi}$ although this is particular to our flow field (which has $\alpha=0.590$ ). A more general relation (which follows via equation (44)) is $T_{\text {trans }}=3 /(4 \alpha)$. The solute mass $M_{\text {trans }}$ at time $T_{\text {trans }}$ is obtained directly via equation (41) and it turns out that $P e^{1 / 2} M_{\text {trans }} \approx 16$ (or more generally it is $4 \sqrt{3 \pi / \alpha}$. We also define a quantity $M_{\text {extrap }}$ by extrapolating the late-time growth of $M$ backwards from $\left(T_{\text {trans }}, M_{\text {trans }}\right)$ until it intersects the $M$-axis. The intersection is always half of $M_{\text {trans }}$, so that $P e^{1 / 2} M_{\text {extrap }} \approx 8$ (or more generally it is $2 \sqrt{3 \pi / \alpha}$ ).

Figure 6(c) compares the computed $P e^{1 / 2} M$ vs $T$ with an approximate formula which uses the "rigid drop" equation (41) up to time $T_{\text {trans }}$, and then uses

$$
M=M_{\text {extrap }}+\dot{M}_{\text {ret,ss }} T
$$

thereafter (with the steady $\dot{M}_{\text {ret,ss }}$ coming from equation (44)). Clearly according to Figure 6(c) the computed $P e^{1 / 2} M$ value and the approximate formula are virtually indistinguishable on the scale of the graph. The laborious procedure for computing $M$ (firstly obtaining $\zeta$ from equation (D.4) and thence $M$ via equations (17) and (D.8)) can be short-cut using this simple explicit $M$ vs $T$ formula.

\subsection{Results for streamline orbit time $T_{\text {orbit }}$ and switching time $T_{\text {switch }}$}

In section 4.3 we computed the amount of solute mass $M$ entering the drop as a function of time T. Another way to represent the results for the amount of solute transferred is in terms of an equivalent volume that solute has invaded. Because of the way that we

\footnotetext{
${ }^{8}$ We refer to this time within the evolution of the boundary layer as a "transition time", so as not to confuse it with the "switching time", at which the boundary layer theory is replaced by a streamlineaveraged theory, a process which happens later on.
} 
normalise our concentration field via equation (3) (zero normalised concentration initially inside the drop and unit normalised concentration outside), it follows (see section 2.3.2) that $M$ and $V$ are actually identical. The specific formula that we employ for $V$ vs $T$ (which is given as equation (D.9) within Appendix D.3) actually corresponds to the prediction for $M$ vs $T$ given in equation (46) with the terms in this equation evaluated via the expressions provided in section 4.3.4.

As we have explained (see section 2.3), the criterion for determining the switching time $T_{\text {switch }}$ between the boundary layer theory and the streamline-averaged one requires matching the time elapsed $T$ with a streamline orbit time $T_{\text {orbit }}$. Accordingly data for $T_{\text {orbit }}$ are considered first in section 4.4 .1 with data for the switching time $T_{\text {switch }}$ itself then being discussed in section 4.4.2.

\subsubsection{Results for $T_{\text {orbit }}$}

Using theory from section 2.3, data from Appendix D.1 and methods from Appendix D.3, we have computed and plotted $T_{\text {orbit }}$ vs streamfunction $\psi$ : see Figure 8(a).

The results are as we expect. The orbit time is a decreasing function of $\psi$. Indeed small $\psi$ values give very long orbit times (since fluid elements are held up near the forward and rear stagnation points of the drop) and a small $\psi$ asymptotic expression (equation (23)) also captures the asymptotic behaviour. Meanwhile the largest $\psi$ values, those neighbouring the internal stagnation point $\psi=\psi_{\text {stag }}$ have the smallest orbit times, approaching a well-defined limit $T_{\text {orbit,stag }}$ (see Appendix D.1.2).

In Figure 8(b) these same $T_{\text {orbit }}$ data are re-expressed as volume $V$ enclosed (between a given streamline and the drop surface) vs $T_{\text {orbit }}$. The volume axis in the plot only extends

up to $V=1$ whereas the full drop volume is $V=\frac{4 \pi}{3}$ : hence Figure 8(b) focuses just on part of the drop corresponding to streamlines close to the surface and/or axis. We plot firstly $V_{\text {orbit }}(T)$ obtained by a small $\psi$ asymptotic formula (equation (26)), but also show the finite $\psi$ correction (equation (24)). Since the focus in Figure 8(b) is only on part of the drop, the finite $\psi$ correction is seen to be small, and is neglected here, as it is easier to work with an analytical expression such as equation (26) provides. 


\subsubsection{Results for the switching time $T_{\text {switch }}$}

Switching between the boundary layer and streamline-averaged theories should occur at a time $T_{\text {switch }}$ when the solute that has entered the drop to date has had the opportunity to orbit exactly once around the volume that it has invaded: this can be found via the intersection between equation (26) for $V$ vs $T_{\text {orbit }}$ and equation (D.9) mentioned earlier for $V$ vs $T$. This latter equation is plotted in Figure 8(b) for selected $P e$ values between $P e=10000$ and $P e=100000$ : recall from sections 1 and 2.1 that typical Peclet numbers for this process are on the order of tens of thousands. All the $T_{\text {switch }}$ intersection points in Figure 8(b) are around ten times longer than the time $T_{\text {trans }}$ defined in section 4.3.4. Hence the volume invaded $V$ vs time elapsed $T$ is definitely in the straight line region (described by equation (D.9)) not the preceding "rigid drop" region (equation (41)).

The intersection points can be read off and are listed in Table 1. Moreover to demonstrate that these intersections all occur in the regime of small $\psi$ (i.e. $\psi \ll \psi_{\text {stag }}$ ), the $\psi$ values from that table are shown as vertical lines in Figure 8(a). What we see in Table 1 is that the volume invaded by solute $V$ (and the value of the streamfunction $\psi$ that manages to enclose this volume) are decreasing functions of $P e$, whereas the time $T_{\text {switch }}$ to switch into the streamline-averaged state is a slowly increasing function of Pe, a fact we already anticipated in section 2.3.2. Remember however that time $T$ has been non-dimensionalised based on a convective time scale, but (again consulting section 2.3.2) a fairer comparison between the behaviours at different $P e$ values is to use a diffusive time scale $t=T / P e$. When expressed in terms of $t$, the time to switch out of the boundary layer regime into the streamline-averaged one is not only exceedingly small $(t \ll 1)$ it is also a decreasing function of $P e$, as we expect and as Ubal et al. (2010) in fact found.

One observation is that we cannot confidently extrapolate the boundary layer theory down to Peclet numbers much smaller than 10000. When $P e=10000$ the boundary layer already accounts for around $17 \%$ of the drop volume by the time when the streamlineaveraged state is attained. For a further reduction in $P e$ down to $P e=1000$ say (a case considered by Ubal et al. (2010)), that volume percentage would increase further still. Although the qualitative picture of a boundary layer state giving way to a streamline- 
averaged one does seem to be borne out by the numerical simulations of Ubal et al. (2010) even for $P e=1000$, the quantitative accuracy of the boundary layer theory might be compromised without a clear separation of scales. This is not a concern however, since as already mentioned, Peclet numbers are more likely to be on the order tens of thousands than on the order of thousands: the boundary layer theory should become more reliable precisely in the regime in which full numerical simulations are very stiff.

In summary, it is possible using the theory we have outlined to estimate when the boundary layer theory must be switched to a streamline-averaged theory. The switch occurs (at least when expressed in terms of time $t$, rather than time $T$ ) at an extremely early time, which becomes earlier still as Peclet number increases. Determining what happens to the system after the switch, requires first of all knowing the parameters in the streamline-averaged equation, specifically the streamline orbit time (which was already considered above) and the effective diffusivity (which is the topic of the next section).

\subsection{Results for effective diffusivity}

Before considering the effective diffusivity itself, it is easier to compute the product $D_{\text {eff }} T_{\text {orbit }}$ which corresponds to the integral on the right hand side of equation (31). This has a well-defined limit as $\psi \rightarrow 0$. The streamline $\psi=0$ covers the drop surface and axis, but on the axis $\sin \theta$ vanishes, and on the surface $r=1$, we find $\left.D_{\text {eff }} T_{\text {orbit }} \rightarrow \zeta_{\text {ss }}\right|_{\theta=\pi}$ where $\left.\zeta_{\mathrm{ss}}\right|_{\theta=\pi}$ is defined by equations (42)-(43). Thus, for the streamline $\psi=0$ passing along the drop surface, the value of $D_{\text {eff }} T_{\text {orbit }}$ (a quantity that is relevant to the streamline-averaged theory) can be related to $\zeta_{\mathrm{ss}}$ (a quantity that arises in the boundary layer theory).

That these two quantities should be related is in fact unsurprising and has already been mentioned in section 2.4. Cross-stream diffusion is responsible both for solute entering the drop at the surface and for solute transferring from streamline to streamline. Crossstream diffusion is moreover faster or slower according to whether streamlines approach one another or separate, with both $\zeta_{\text {ss }}$ and $D_{\text {eff }} T_{\text {orbit }}$ being measures of this.

Although $D_{\text {eff }} T_{\text {orbit }}$ is finite on the drop surface, at the opposite end of the $\psi$ domain, namely at the internal stagnation point $\psi \rightarrow \psi_{\text {stag }}$, it vanishes: this is clear from equation (31), since both the integrand and the integration path length within that equation 
vanish in the $\psi \rightarrow \psi_{\text {stag }}$ limit. We expect then that $D_{\text {eff }} T_{\text {orbit }}$ will be a decreasing function of $\psi$ and Figure 9 (a) which plots $D_{\text {eff }} T_{\text {orbit }}$ over the entire $\psi$ domain bears out this expectation. The striking observation from Figure $9(\mathrm{a})$ is that $D_{\text {eff }} T_{\text {orbit }}$ is very nearly a straight line function of $\psi$ implying that

$$
\left.D_{\text {eff }} T_{\text {orbit }} \approx\left(1-\psi / \psi_{\text {stag }}\right) \zeta_{\text {ss }}\right|_{\theta=\pi}
$$

Combining the $T_{\text {orbit }}$ data from Figure $8(\mathrm{a})$ with the $D_{\text {eff }} T_{\text {orbit }}$ data from Figure 9 (a) provides $D_{\text {eff }}$ vs $\psi$ which is plotted in Figure $9(\mathrm{~b})$. Observe that $D_{\text {eff }}$ vanishes at both ends of the $\psi$ interval: at one end of the interval $D_{\text {eff }} T_{\text {orbit }}$ is finite, but $T_{\text {orbit }}$ is infinite, whereas at the other end, $T_{\text {orbit }}$ is finite but $D_{\text {eff }} T_{\text {orbit }}$ vanishes. It follows then that $D_{\text {eff }}$ has a local maximum in the interior of the interval. However on the approach to $\psi \rightarrow 0$, we would have to go to exceedingly tiny $\psi$ values in order to attain a small $D_{\text {eff }}$ value. This follows from $D_{\text {eff }} T_{\text {orbit }}$ being finite, but $T_{\text {orbit }}$ having a very weak logarithmic divergence.

This completes our discussion of the functions $T_{\text {orbit }}, D_{\text {eff }}$ and their product $D_{\text {eff }} T_{\text {orbit }}$. Having evaluated these functions we are now ready to obtain results from streamlineaveraged equations, which we do in the next section.

\subsection{Results for streamline-averaged theory}

This section is organised as follows. Sections 4.6.1-4.6.2 show results specific to the streamline-averaged theory (specifically the spatiotemporal behaviour of the streamlineaveraged solute mass fraction $W$ ), whilst sections 4.6.3-4.6.5 give overall predictions for mass transfer. These overall results incorporate both the boundary layer and streamlineaveraged regimes, but as we will see, the latter regime dominates most of the evolution.

\subsubsection{Streamline-averaged profiles for $W$}

Profiles of $W$ (the streamline-averaged mass fraction) vs streamfunction $\psi$ at various times $t$ are presented in Figure 10(a). Note that the data in Figure 10(a) are nominally for $P e=10000$. Recall however that $P e$ does not affect the streamline-averaged theory itself, merely the time at which the streamline-averaged state is first achieved. This particular time, and the $W$ vs $\psi$ profile relevant to it (and also the amount of solute mass under 
that profile), are obtained via the theory discussed in sections 2.3 and 2.5.1. According to Table 1 , for $P e=10000$, the streamline-averaged state is achieved at $T=10.37$ and hence at $t \equiv T / P e \approx 0.001$. All the $W$ vs $\psi$ profiles shown in Figure 10(a) are for times significantly later than this, so correspond to a system already in the streamline-averaged state (and thus insensitive to $\mathrm{Pe}$ ).

What we see in Figure 10(a) is that solute (which transfers from streamline to streamline diffusively) gradually invades more and more of the drop. What is evident however is that for time $t=0.002$ solute has still not reached as far as the internal stagnation point of the drop $\psi=\psi_{\text {stag }}$, and for $t=0.01$ solute it is only just beginning to arrive there. In fact the boundary condition (33) only permits $W$ to grow with time at $\psi=\psi_{\text {stag }}$ when non-zero spatial gradients $\partial W / \partial \psi$ have also arrived there.

As time proceeds, the spatial variations in $W$ decrease, meaning that the concentration driving force (highest concentration in the spatial domain less lowest concentration at any given $t$ ) also decays with time. The model of Uribe-Ramirez and Korchinsky (2000b) also incorporates the decay of concentration driving forces, but assumes that the entire concentration difference is realised across a very narrow boundary layer of thickness order $P e^{-1 / 2}$ near the drop surface. Here however we see that the concentration differences only start to decay once the concentration gradients manage to spread across the full $\psi$ domain i.e. across the entire streamline pattern. By spreading the concentration gradients out in this fashion, instead of confining them to a thin layer, the decay in the concentration driving force becomes now much slower. Nonetheless the concentration driving force does decay, and by time $t=0.1$ we see that the concentration field is approaching uniformity, with the concentration driving force being only around $10 \%$ of its original value. The smaller spatial gradients in this near uniform field, again however imply, via boundary condition (33), smaller temporal rates of change for $W$ near the stagnation point.

\subsubsection{Streamline-averaged evolution for $W$}

Additional insights can be obtained by plotting data for $W$ vs $t$. We do this in Figure 10(b) both for the internal stagnation point $\psi=\psi_{\text {stag }}$ (the point that is slowest to evolve) and for a volume-average $W$ over the entire drop. It is interesting just how 
dissimilar the two curves are.

The volume-averaged $W$ starts off from a non-zero value, owing to the fact that the streamline-averaged theory does not extend all the way down to zero time, but instead inherits an initial condition from the boundary layer theory. For our nominal Peclet number $P e=10000$ the non-zero starting value for volume-averaged $W$ is the ratio between the volume considered to be invaded by solute during the boundary layer period (read off from Table 1) and the full volume $\left(\frac{4 \pi}{3}\right)$ of the drop. The rate of change of the volume-averaged $W$ also starts off being rapid, since the streamline-averaged model inherits a solute concentration field containing initially sharp gradients from the boundary layer theory that precedes it. However the growth of volume-averaged $W$ slows down, first of all as the solute concentration gradients spread themselves over increasing numbers of streamlines, and following that as the concentration driving force (highest concentration in the spatial domain less lowest concentration in the domain) decays.

By contrast $W$ at $\psi=\psi_{\text {stag }}$ starts off effectively from zero, and does not even begin to increase until the drop is already half filled with solute (i.e. volume-averaged $W$ equal to 0.5). Growth of $\left.W\right|_{\psi=\psi_{\text {stag }}}$, after it starts, is initially slow, then speeds up for a period, and subsequently slows down again: owing to boundary condition (33), this temporal behaviour reflects also how the spatial concentration gradient at $\psi=\psi_{\text {stag }}$ behaves.

\subsubsection{Overall predictions for mass transfer spanning different regimes}

Our overall aim in developing the streamline-averaged theory and matching it to the boundary layer theory was to obtain a means to compute the solute transfer into a circulating drop over the full time domain of evolution whilst avoiding having to solve the highly stiff partial differential equations that ordinarily result in the high Peclet number limit. In order to demonstrate how we have addressed this aim we plot in Figure 11 the predictions of our calculations for total solute mass $M$ in the drop vs time $t$ spanning both the boundary layer regime and the streamline-averaged one.

Figure 11(a) shows data only up as far as $t=0.002$ (which is long before the mass transfer process is complete). Nonetheless the range of times is sufficient to examine the details of switching between the boundary layer and streamline-averaged states. What we 
see is that at very early times the solute mass vs time follows a rigid drop formula, but this soon gives way to a steady rate of increase, still within the boundary layer regime. The larger the $P e$ value, the sooner the steady rate of increase is attained, and the steeper the rise during the steady rate period is. As mentioned in section 2.5.1 however, the rate of increase of solute mass suddenly drops when the streamline-averaged state is attained.

Mathematically this is due to the way that we implement the initial condition for the streamline-averaged equation redistributing the available solute from a state given by equation (11) (which favours solute being towards the rear part of the drop for any given streamline) to a state given by equation (34) (which treats all points on any given streamline uniformly). Values of $\psi_{*}$ (the parameter appearing within equation (34) that governs the initial streamline-averaged state) are reported in Table 1: as anticipated in sections 2.5.1 they are decreasing functions of Peclet number Pe.

We can use these $\psi_{*}$ values to determine how much $\mathrm{d} M / \mathrm{d} t$ changes upon switching into the streamline-averaged state. Before the switch we have $\mathrm{d} M /\left.\mathrm{d} t\right|_{\text {before }}=P e \dot{M}_{\text {ret,ss }}$ where the prefactor $P e$ comes from writing the derivative in terms of $t$ rather than $T$, and where $\dot{M}_{\text {ret,ss }}$ is given by equations (44)-(45). Immediately after the switch we appeal to equation (18), but with $\partial w /\left.\partial \psi\right|_{\psi=0}$ given by $-2 /\left(\sqrt{\pi} \psi_{*}\right)$ (see equation (34)) and not by equation (19). Hence

$$
\left.\frac{\mathrm{d} M}{\mathrm{~d} t}\right|_{\mathrm{after}}=\left.2 \pi \frac{2}{\sqrt{\pi} \psi_{*}} \zeta_{\mathrm{ss}}\right|_{\theta=\pi} .
$$

The data for $\psi_{*}$ presented in Table 1 turn out to give $\mathrm{d} M /\left.\mathrm{d} t\right|_{\text {after }}$ being around 0.51 times $\mathrm{d} M /\left.\mathrm{d} t\right|_{\text {before }}$ regardless of the value of Peclet number.

An instantaneous change in $\mathrm{d} M / \mathrm{d} t$ as is envisaged here is of course artificial. Nonetheless (see e.g. section 2.5.1) the full numerical simulations of Ubal et al. (2010) show an abrupt decrease in $\mathrm{d} M / \mathrm{d} t$ over a small but finite period of very limited duration. This reflects the fact that as soon as fluid elements have circulated once around the drop, it is no longer possible to bring fresh fluid elements from the drop interior into contact with the surface, concentration gradients thereby fall and the mass transfer rate must suffer. 


\subsubsection{Overall predictions on longer time scales}

Figure 11(b) shows the same data as Figure 11(a) but on a much longer time scale. We use a logarithmic time scale here which stretches out the initial boundary layer phase of evolution in order to make it visible. The overwhelming majority of the evolution of the concentration field is spent in the streamline-averaged state, with the boundary layer regime estimated to account for only about $1 \%$ of the time domain (when $P e=10000$ ) or even as little as $0.1 \%$ (in the case of $P e=100000$ ). These estimates follow because Table 1 shows the boundary layer state surviving in very rough terms on the order of 10 units of $T$ (i.e. 10/Pe units of $t$ ) whereas we know from Figure 10(a) that the streamlineaveraged state requires on the order of 0.1 time units to complete. Furthermore the solute mass transfer achieved during the boundary layer phase follows from equation (46) or equivalently from equation (D.9) (remembering that solute mass $M$ is identical to invaded volume $V$ in our scalings). The mass given by these equations scales as $P e^{-1 / 2}$ and so for large $P e$ is necessarily a small fraction of the total amount of solute mass transferred during the overall process (which in this normalised system is $\frac{4 \pi}{3}$ corresponding to a unit concentration filling the volume of the unit sphere). So the streamline-averaged state (and not the boundary layer state) very definitely dominates the overall evolution. Another important observation we make from Figure 11(b) is that with increasing time, the streamline-averaged evolution outperforms the rigid drop: as mentioned in section 1, it is faster for mass to diffuse across streamlines to reach an internal stagnation point, than to diffuse the even greater distance from outside the drop all the way to its centre.

One additional comparison we make is between the streamline-averaged theory presented here and the predictions of Uribe-Ramirez and Korchinsky (2000b). Recall (see sections 1, 2.6 and 4.6.1) that these latter predictions assume that that fluid returned from the boundary layer to the bulk of the drop mixes well with fluid already there, such that the structure of the boundary layer is preserved indefinitely, but the driving force across that boundary layer decays as more and more mass transfer takes place. The resulting 
formula for solute mass $M$ vs time $t$ (which we denote $M_{\text {Uribe }}$ ) is given by

$$
M_{\text {Uribe }}=\frac{4 \pi}{3}\left(1-\exp \left(-\frac{3 \dot{M}_{\mathrm{ret}, \mathrm{ss}}}{4 \pi} T\right)\right)=\frac{4 \pi}{3}\left(1-\exp \left(-\frac{3 P e \dot{M}_{\mathrm{ret}, \mathrm{ss}}}{4 \pi} t\right)\right)
$$

where $\dot{M}_{\text {ret,ss }}$ is defined by equation (44). Predictions from (49) are plotted in Figure 11(c), and can be compared with the predictions from streamline-averaged theory.

Clearly the predictions of equation (49) reach steady state much more rapidly than the streamline-averaged ones do. Mathematically this is a result of $\dot{M}_{\text {ret,ss }}$ being an order $P e^{-1 / 2}$ quantity (via equation (44)) and hence $P e \dot{M}_{\text {ret,ss }}$ within equation (49) being order $P e^{1 / 2}$. Physically this is saying that, by assuming a thin boundary layer structure is retained indefinitely, the time scale for achieving transfer of solute mass is significantly compressed in the predictions of Uribe-Ramirez and Korchinsky (2000b) compared to what a streamline-averaged theory would predict.

Finally we note that there is a qualitative similarity between the streamline-averaged data presented in Figure 11(b) and/or Figure 11(c), and the full numerical simulation data plotted in a similar format in Ubal et al. (2010). Unfortunately we cannot compare the two sets of data directly because Ubal et al. (2010) considered a multi-component diffusion system with coupling between the diffusion of different components, whereas here single component diffusion is considered. Note also that there is limited overlap between the ranges of Peclet number considered: Ubal et al. (2010) only considered $P e$ up to 10000 , whereas the data presented here start from $P e=10000$ upwards: as mentioned in section 4.4, if we push to Peclet numbers smaller than this we lose the separation of scales upon which the boundary layer model is based. Quantitative comparison of our model predictions with full numerical simulations (specifically single-component diffusion with $P e$ of 10000 or greater) remains an important outstanding item of future work.

\subsubsection{Analysis of Sherwood numbers}

Yet another way to analyse the data is in terms of Sherwood numbers. Here we define the Sherwood number $S h$ to be the ratio of the rate of change of solute mass $\mathrm{d} M / \mathrm{d} t$ to the characteristic rate of change associated with diffusion alone. In our dimensionless system, 
we estimate the diffusion only contribution as the concentration driving force (one minus the volume averaged $W$ ) multiplied by the surface area of the unit sphere $4 \pi$. Since the volume averaged $W$ is the ratio of $M$ and $\frac{4 \pi}{3}$ it follows

$$
S h=\frac{\mathrm{d} M / \mathrm{d} t}{4 \pi\left(1-\frac{3 M}{4 \pi}\right)} .
$$

This definition for $S h$ is half of that of Edelmann et al. (2017) since drop radius (not drop diameter) is chosen as the characteristic length scale for non-dimensionalising lengths.

Data for $S h$ vs $t$ are presented in Figure 12. Examining Sherwood numbers is actually a very robust test of any model, because $S h$ is highly sensitive to model predictions as it involves not just $M$ but also $\mathrm{d} M / \mathrm{d} t$. Figure 12 shows that the data follow a "rigid drop" formula for $S h$ at very early times. Immediately after the predictions start to depart from the rigid drop, but provided the boundary layer theory still applies, $S h$ is roughly constant. We have shown this as a horizontal line in Figure 12, on the grounds that $M \ll 1$ in this time domain and hence $S h \approx(4 \pi)^{-1} \mathrm{~d} M / \mathrm{d} t$ with $\mathrm{d} M / \mathrm{d} t$ being constant and equal to $P e \dot{M}_{\text {ret,ss }} \approx 2 \pi P e^{1 / 2}$ according to equation (45): clearly $S h$ is sensitive to $P e$ during this period of the evolution. Once the streamline-averaged state is achieved, the Sherwood number undergoes a discontinuous step change so as to attain the streamlineaverage $S h$ vs $t$ curve. This step change is an artifact of the model associated with assuming a redistribution of the solute mass at the instant that the streamline-averaged state is attained: it is expected that one would actually see a rapid but not instantaneous decrease in $S h$ around this time. In the streamline-averaged state $S h$ is no longer sensitive to $P e$, but nonetheless the streamline-averaged $S h$ exceeds the rigid drop one including in the limit of arbitrarily large time: for the rigid drop $S h$ can be shown to asymptote to $\pi^{2} / 3$ whereas numerically our streamline-averaged data are found to approach $S h \approx 8.6$.

\section{Conclusions}

We have presented a technique for predicting the time evolution of solute mass transfer into a circulating drop in the context of liquid-liquid extraction. This is a challenging system upon which to perform full numerical simulations: despite the fact that the full 
numerical simulations are conceptually easy to set up, owing to the Peclet number being exceedingly large (on the order of tens of thousands), the governing equations are stiff. We therefore sought to find a technique to solve for the evolution of the solute mass fraction in the drop which does not require the solution of such stiff equations.

The model presented here recognises that the drop spends most of its lifetime in a streamline-averaged state, such that solute concentration varies across streamlines but not along them. Mass transfer in this regime proceeds in the cross-stream direction and is controlled by diffusion despite the very large Peclet number: although convection is rapid, by definition it only transports solute along streamlines, not in a cross-stream direction.

Convection in this regime manifests itself only in the sense that it can either bring streamlines together or separate them, and cross-stream diffusion proceeds much faster across more closely spaced streamlines. Information from the flow field is therefore incorporated by assigning an effective diffusivity to each streamline. This can be thought of as being a time average moving along the streamline of the inverse square of streamline separation distance, a measure which is relevant because the rate of diffusion rises in this fashion if streamline separation distances fall.

The fluid elements in the drop circulate around many times whereas those outside the drop flow past it once only and (when Peclet number is large) flow past quickly. The implication is that mass transfer has sufficient time to develop over much larger length scales inside the drop than out, suggesting that it is relevant, as we have done here, to solve an internal problem for which, as an approximation, the solute concentration on the drop surface is kept fixed: ordinarily internal problems are considered when the internal diffusivity is substantially less than the external one, but it is not even necessary to assume that provided the internal length scale is substantially greater than the external one. As time proceeds, the mass transfer in this internal problem extends all the way from the drop surface and axis to an internal stagnation point about which the flow pattern in the drop circulates. This is a rather smaller distance than that from the drop surface to the drop centre, suggesting that streamline-averaged diffusion proceeds more quickly than the "rigid drop" diffusion that would occur in the absence of any circulation whatsoever: this 
expectation is indeed borne out by our numerical data for the streamline-averaged model.

Although the streamline-averaged state accounts for the overwhelming majority of the lifetime of the evolution of the drop, it does not apply right down to the initial state. At relatively early times, prior to one complete streamline circulation having been executed, the system cannot be in a streamline-averaged state. Considering for instance, a circulation streamline that passes both close to the drop surface and along the drop axis, the solute concentration on those fluid elements exposed to the surface must be very different from the concentration on those elements upon the same streamline which have spent all their lifetime to date near the drop axis but deep in the interior. Such a regime needs to be described by a boundary layer model and, unlike the streamline-averaged model, it evolves on a (fast) convective time scale rather than a (slower) diffusive one.

A key variable appearing in the boundary layer description is a so called "extent of diffusion" function. Once this function is known, the amount of solute in the drop can be found very straightforwardly. It was shown that the extent of diffusion is an analogue of the aforementioned effective diffusivity, except that it applies specifically on the drop surface rather than throughout the entire drop. It behaves like a "diffusive clock" which is convected along the surface growing quickly when streamlines near the surface move close together but slowing down as streamlines move apart. Increases in the value of the extent of diffusion imply solute penetrating across increasing numbers of streamlines.

Although convection is an integral part of how the extent of diffusion behaves, at very early times, when the boundary layer is arbitrarily thin, diffusion invariably dominates. The result is that the boundary layer initially adopts a spherically symmetric configuration, just as would happen in a rigid drop without any convection. As time progresses however, the role of convection starts to become important, and this causes the extent of diffusion function (and likewise the distribution of solute mass which follows from it) to become skewed towards the rear of the drop.

Solute enters the drop through its surface and is carried by the circulation along a boundary layer towards the rear, and subsequently is returned by the circulation into the interior of the drop. The extent of diffusion (and hence the structure of the boundary 
layer) evolves towards a steady state but with the steady state on the forward part of the drop being approached considerably faster than at the rear. Since most of the mass transfer across the surface actually happens through the forward end, the boundary layer model predicts that the rate of growth of solute mass in the drop attains a near steady state surprisingly quickly. Moreover the higher the Peclet number, the faster the rate of growth of solute mass during this period, assuming that the comparison is made using a diffusive rather than a convective time scale.

Of course this steady state rate of growth predicted by the boundary layer model cannot proceed indefinitely as the system switches into a streamline-averaged state at longer times. Intuitively the time at which this switch occurs should correspond to one streamline orbit time for those particular streamlines in the drop that pass near the drop surface and axis. At that time, large gradients in solute concentration along such streamlines must cease, since all fluid elements currently on such a streamline have already been exposed to close proximity to the surface at some point during their evolution.

Although this criterion is easy to describe intuitively, it is less easy to formulate mathematically, because the streamline orbit time actually diverges as the drop surface is approached, owing to there being stagnation points at the forward and rear of the drop. It is necessary therefore to use the boundary layer theory to track the volume that solute invades as time proceeds, until the streamline orbit time corresponding to this invaded volume matches the time elapsed. Using this criterion, predictions for the switching time between the boundary layer state and the streamline-averaged one then indicate that increasing Peclet number leads to the switch happening earlier both in terms of time (again with time being measured here on a diffusive scale rather than a convective one) and in terms of net amount of solute transferred up to the switch.

At the switching time, an initial condition for the streamline-averaged equations must be implemented. Our model assumes that this can be done by redistributing the solute mass inherited from the preceding boundary layer model. Redistributing solute in this fashion, leads to an instantaneous change (specifically a step decrease) in the rate at which solute enters the drop immediately after that. Although artificial, we view this 
instantaneous change in the rate of mass transfer as an idealization of an abrupt decrease in the mass transfer rate that actually occurs over a small but finite time (associated, as mentioned earlier, with all the fluid elements near the surface of the drop having orbited once around already, and thereby having had prior contact with solute at the surface).

Taken together, the boundary layer theory, the streamline-averaged theory, and the criterion developed here for switching between the former theory and the latter, are able to make predictions for the solute contained in the drop for any chosen Peclet number (assuming Peclet number is large) and for the full time domain of evolution of the mass transfer. The time to achieve steady state according to the streamline-averaged theory is substantially less than what a rigid drop model (without circulation) would predict. In addition, the time to steady state is substantially more than the predictions of Uribe-Ramirez and Korchinsky (2000b) (which are based on assuming a sharp boundary layer continues to persist even after many streamline orbits have been executed). Although the predictions of the model developed here do seem to reproduce qualitative features of literature data obtained from full numerical simulations of the (exceedingly stiff) advection-diffusion equations, we emphasise that we have not yet subjected the model predictions to a quantitative comparison against full numerical simulation data. Doing such a comparison is identified as an exceedingly important item for further work.

\section{Appendix A. Obtaining the asymptotic expression for streamline orbit time}

It was claimed in the main text that streamlines passing close to the drop surface and/or drop axis have orbit times $T_{\text {orbit }}$ that satisfy equation (23), namely $T_{\text {orbit }} \sim$ $A \log (1 / \psi)+B$ where $\psi$ is the streamfunction (which vanishes on the drop surface and axis) and where $A$ and $B$ are constants. This appendix explains how this relation can be obtained, and how to go about determining the constants $A$ and $B$. This is achieved by first determining how long a fluid material element is held up near the forward and rear stagnation points of the drop, and subsequently adding in the times to displace from the neighbourhood of one stagnation point to the neighbourhood of the other.

In order to compute orbit times, we require a surface velocity field $\left.u_{\theta}\right|_{\text {surf }}$ (varying 
with respect to $\theta$, and vanishing at $\theta=0$ and $\theta=\pi$ ) and an axis velocity field $\left.u_{r}\right|_{\text {axis }}$ (varying with respect to $r$, vanishing at $r=1$, and generally with a different functional form on the upper part of the axis, $\theta=0$, and the lower part of the axis, $\theta=\pi$ ). At the stagnation points, $r=1$ and either $\theta=0$ (forward) or $\theta=\pi$ (rear), local strain rates can be computed in terms of derivatives of $\left.u_{\theta}\right|_{\text {surf }}$ and/or $\left.u_{r}\right|_{\text {axis }}$, and the local forms of the streamfunctions are then known: they correspond to a uniaxial compression (and hence a biaxial extension) near the forward stagnation point and a uniaxial extension (and hence a biaxial compression) near the rear stagnation point. Using these known forms, it turns out that locally near the forward stagnation point $\psi$ reduces to $E_{\text {biax }} \theta^{2}(1-r)$ (where $E_{\text {biax }}$ is the biaxial extension rate, a constant of order unity in our dimensionless system of equations) and near the rear stagnation point it reduces to $\frac{1}{2} E_{\text {uniax }}(\pi-\theta)^{2}(1-r)$ (where $E_{\text {uniax }}$ is the uniaxial extension rate, again a constant of order unity in our dimensionless system). It is easy to demonstrate by substituting these functional forms into equations (4)-(5) that uniaxial compression or uniaxial extension velocity fields result locally.

We perform an analysis near the forward stagnation point as follows. We select a streamline inside the drop with a small $\psi$ value $(\psi \ll 1)$ ensuring that the streamline passes close to the stagnation point. We choose two points on the streamline, one just upstream of the forward stagnation point $\left(r_{\text {up fwd }}, \theta_{\text {up,fwd }}\right)$ and one just downstream of it $\left(r_{\text {down,fwd }}, \theta_{\text {down,fwd }}\right)$. We aim to determine the time to migrate between these points.

Moreover we select an $r_{\text {up,fwd }}$ value such that $\psi^{1 / 3} \ll 1-r_{\text {up,fwd }} \ll 1$ and we also choose a $\theta_{\text {down,fwd }}$ value such that $\psi^{1 / 3} \ll \theta_{\text {down,fwd }} \ll 1$. The local functional form of the streamlines implies that $\theta^{2}(1-r)$ is conserved on the streamline, and owing to the way in which we have constrained $r_{\text {up,fwd }}$, we can deduce that $\theta_{\text {up,fwd }} \ll 1-r_{\text {up,fwd }}$. This means that the point $\left(r_{\text {up,fwd }}, \theta_{\text {down,fwd }}\right)$ is far closer to the drop axis than it is to the drop surface. Analogously the constraint we have placed on $\theta_{\text {down,fwd }}$ implies $1-r_{\text {down,fwd }} \ll$ $\theta_{\text {down,fwd }}$, so that $\left(r_{\text {down,fwd }}, \theta_{\text {down,fwd }}\right)$ is far closer to the drop surface than it is to the axis. Thus migration from $\left(r_{\mathrm{up}, \mathrm{fwd}}, \theta_{\mathrm{up}, \mathrm{fwd}}\right)$ to $\left(r_{\mathrm{down}, \mathrm{fwd}}, \theta_{\mathrm{down}, \mathrm{fwd}}\right)$ corresponds in effect to migrating from the drop axis to the drop surface whilst passing close to the stagnation point. Using the local form of the streamfunction $\left(\psi \sim E_{\text {biax }} \theta^{2}(1-r)\right)$ and the velocity 
fields one derives from it (i.e. biaxial extension near a stagnation point), it is possible to determine analytically via elementary calculus the time to move from $\left(r_{\mathrm{up}, \mathrm{fwd}}, \theta_{\mathrm{up}, \mathrm{fwd}}\right)$ to $\left(r_{\text {down,fwd }}, \theta_{\text {down,fwd }}\right)$ for any chosen streamline $\psi$ and this is what leads to the logarithmic term in equation (23). Moreover the larger the value of $E_{\text {biax }}$, the faster the motion, and the shorter the hold up time near the stagnation point: this manifests itself in the prefactor multiplying the logarithmic term.

Similar considerations apply to computing the hold up time near the rear stagnation point, with a $\psi \ll 1$ streamline inside the drop being chosen, and with suitably chosen upstream and downstream points $\left(r_{\text {up,rear }}, \theta_{\text {up,rear }}\right)$ and $\left(r_{\text {down,rear }}, \theta_{\text {down,rear }}\right)$ being defined, both located near the rear stagnation point, but with the upstream point chosen to be much closer to the surface than it is to the axis, and the downstream point chosen to be much closer to the axis than to the surface. Again a logarithmic term results, and again there is a prefactor that scales inversely with the strain rate ( $E_{\text {uniax }}$ in this case). Hence the total times that fluid elements on a given streamline are held up near both forward and rear stagnation points are well defined. Intuitively, the smaller the value of $\psi$, the closer a streamline passes to either stagnation point (forward or rear), and the longer the fluid is held up near these stagnation points, which is what equation (23) predicts.

The total orbit time is obtained adding in the (finite) times taken to traverse the drop away from the stagnation points i.e. from $\theta=\theta_{\text {down,fwd }}$ to $\theta=\theta_{\text {up,rear }}$ along the drop surface (equation (9) is applicable here) as well along the drop axis $\theta=\pi$ from $r=r_{\text {down,rear }}$ to the drop centre, and additionally along the drop axis $\theta=0$ from the drop centre to $r=r_{\text {up,fwd }}, \theta=0$ : these can be readily obtained by quadrature if necessary. An expression of the form of equation (23) then results.

\section{Appendix B. Relation between enclosed volume, orbit time \& streamfunction}

Equation (24) in the main text claimed that there was a relation between volume $V$ enclosed by a set of streamlines, the streamline orbit time $T_{\text {orbit }}$ and the streamfunction. In this appendix we explain how this relation comes about.

We consider a generalised coordinate system where $s$ measures distance along a stream- 
line, $\psi$ represents the streamfunction, and $\phi$ is the azimuthal angle. As in the main text (section 2.4), we can replace the coordinate $s$ by a new coordinate $S$ where $S=s / L_{\text {orbit }}(\psi)$ where $L_{\text {orbit }}(\psi)$ denotes the length of the streamline orbit: this now gives us a coordinate set $S, \psi$ and $\phi$. Following the procedure outlined in section 2.4 we can also construct an orthogonal coordinate set $\mathcal{S}, \psi$ and $\phi$ such that $\mathcal{S}=S$ on one selected streamline (but not, in general, on any others). Nonetheless the relation between $\mathcal{S}$ and $S$ can be readily determined via a geometric construction (see section 2.4).

Computing the volume of a fluid element is simple using the orthogonal $\mathcal{S}, \psi$ and $\phi$ coordinate set: the volume depends on the product of three scale factors, one associated with each coordinate. Remembering that $s$ is a distance variable, and that $S=s / L_{\text {orbit }}$, it follows that the scale factor associated with $\mathcal{S}$ works out as $\chi L_{\text {orbit }}$ where $\chi \equiv(\partial S / \partial \mathcal{S})_{\psi}$. Meanwhile $\psi$ only varies in the direction normal to streamlines and (via equations (4)-(5))

$$
|\nabla \psi|=\left(\frac{1}{r^{2}}\left(\frac{\partial \psi}{\partial \theta}\right)^{2}+\left(\frac{\partial \psi}{\partial r}\right)^{2}\right)^{1 / 2}=r \sin \theta\left(u_{r}^{2}+u_{\theta}^{2}\right)^{1 / 2}=u_{s} r \sin \theta
$$

where $u_{s}$ denotes the flow speed along a streamline. Hence the scale factor associated with $\psi$ is $|\nabla \psi|^{-1}=\left(u_{s} r \sin \theta\right)^{-1}$. Finally the scale factor associated with $\phi$ is $r \sin \theta$.

The product of all three scale factors is therefore $\chi L_{\text {orbit }} / u_{s}$, so the enclosed volume is

$$
V=2 \pi \int_{0}^{\psi} \oint \mathrm{d} \psi \mathrm{d} \mathcal{S} \chi L_{\text {orbit }} / u_{s}=2 \pi \int_{0}^{\psi} \oint \mathrm{d} \psi \mathrm{d} s / u_{s}
$$

the factor $2 \pi$ coming from integrating over the azimuthal coordinate $\phi$. Substituting $T_{\text {orbit }}=\oint \mathrm{d} s / u_{s}$ from equation (22) into equation (B.2), we obtain equation (24).

\section{Appendix C. Boundary condition at the internal stagnation point}

This appendix proves the validity of equation (33) that gives the boundary condition for the streamline-averaged system at the internal stagnation point.

Consider first of all the situation in physical space. Suppose the internal stagnation point is at coordinate location $r_{\text {stag }}$ and $\theta_{\text {stag }}$. Choose a particular streamline orbit close to the internal stagnation point. Suppose that the streamline encloses a small area $A_{\text {orbit }}$. 
By analysing the flow field in the neighbourhood of the internal stagnation point, which locally is comprised of a strain rate field and a vorticity field, it is possible to show that the streamline has an elliptical shape with semi-major and semi-minor axes, respectively denoted $a_{ \pm}$, that satisfy

$$
a_{ \pm}=\left(1 \pm 2 E_{\text {stag }} / \omega_{\text {stag }}\right)^{1 / 2}\left(1-4 E_{\text {stag }}^{2} / \omega_{\text {stag }}^{2}\right)^{-1 / 4}\left(A_{\text {orbit }} / \pi\right)^{1 / 2}
$$

where $\pm E_{\text {stag }}$ are eigenvalues of the strain rate tensor and $\omega_{\text {stag }}$ is the vorticity, both evaluated at the internal stagnation point. Likewise it is possible to show that the streamline enclosing an area ${ }^{9} A_{\text {orbit }}$ corresponds to a $\psi$ value

$$
\psi=\psi_{\text {stag }}-\left(1-4 E_{\text {stag }}^{2} / \omega_{\text {stag }}^{2}\right)^{1 / 2} \frac{A_{\text {orbit }} \omega_{\text {stag }}}{4 \pi} r_{\text {stag }} \sin \theta_{\text {stag }}
$$

where $\psi_{\text {stag }}$ is the (maximal) value of the streamfunction at the stagnation point itself. That the difference in streamfunction $\psi_{\text {stag }}-\psi$ is related to the enclosed area $A_{\text {orbit }}$ is unsurprising since close to the internal stagnation point the streamfunction must exhibit second order variation with position, while the enclosed area is also a second order quantity with respect to position. Equation (C.2) in fact follows from equation (24) recognising that as $\psi \rightarrow \psi_{\text {stag }}$, the value of $T_{\text {orbit }}$ attains a well-defined finite limiting value (the decreases in speed on the approach to the stagnation point being compensated for by decreases in the path length around the ellipse) and moreover an enclosed area $A_{\text {orbit }}$ in the $(r, \theta)$ plane implies a well-defined enclosed volume in spherical polar $(r, \theta, \phi)$ coordinates.

All the results presented to date in this appendix, follow just from the geometry of the streamfunction field in the neighbourhood of the stagnation point $\psi=\psi_{\text {stag. }}$. We now need to couple these results to expressions for mass transfer, in order to develop a boundary condition for the streamline-averaged mass transfer theory.

We proceed as follows. We equate the rate of increase of mass inside the orbit $A_{\text {orbit }}$

\footnotetext{
${ }^{9}$ Previously we have measured enclosed volume starting from the drop surface and axis moving into the drop. For the present argument in the neighbourhood of the internal stagnation point however, we measure enclosed area from the internal stagnation point moving outwards.
} 
to the mass flux across the boundary of $A_{\text {orbit }}$. Hence for a streamline-averaged solute mass fraction field $W$

$$
A_{\text {orbit }} \partial W / \partial t=-\oint \partial W / \partial n \mathrm{~d} s
$$

where $n$ is a distance coordinate measured from the boundary of orbit $A_{\text {orbit }}$ pointing inwards, with $s$ being a distance coordinate measured along the orbit. Note that for the problem of interest, i.e. mass transfer into the drop, $W$ tends to decrease as $n$ increases, and hence $W$ increases with time, consistent with the notion of mass diffusing from the drop surface and axis to the internal stagnation point. We now write $\partial W / \partial n$ as

$$
\partial W / \partial n=\partial W / \partial \psi \partial \psi / \partial n
$$

We expect $\partial W / \partial \psi$ to be negative, whereas $\partial \psi / \partial n \approx r_{\text {stag }} \sin \theta_{\text {stag }} u_{s}$ (where $u_{s}$ is the speed at any point on the streamline). Under the assumption that $\partial W / \partial \psi$ is uniform across the entire area $A_{\text {orbit }}$ we deduce

$$
A_{\text {orbit }} \frac{\partial W}{\partial t}=-\frac{\partial W}{\partial \psi} r_{\text {stag }} \sin \theta_{\text {stag }} \oint u_{s} \mathrm{~d} s
$$

Stokes theorem however implies that

$$
\oint u_{s} \mathrm{~d} s=\omega_{\text {stag }} A_{\text {orbit }}
$$

where recall $\omega_{\text {stag }}$ is the vorticity at the stagnation point. Terms in $A_{\text {orbit }}$ can thus be cancelled from both sides of equation (C.5) and a limit taken such that $A_{\text {orbit }} \rightarrow 0$. Equation (33) as given in the main text then results.

In the above we used physical arguments to derive the boundary condition (33). Previous work (Brignell, 1975; Prakash and Sirignano, 1978) used mathematical arguments instead. Given that $D_{\text {eff }} T_{\text {orbit }}$ vanishes at the internal stagnation point, and assuming that $\partial^{2} W / \partial \psi^{2}$ remains finite there, equation (32) reduces to

$$
\partial W /\left.\partial t\right|_{\text {stag }}=T_{\text {orbit,stag }}^{-1} \partial\left(D_{\text {eff }} T_{\text {orbit }}\right) /\left.\partial \psi\right|_{\text {stag }} \partial W /\left.\partial \psi\right|_{\text {stag }}
$$


By inspection, this is seen to have the same general mathematical form as equation (33) and after some mathematical manipulation can be shown to reduce to it.

\section{Appendix D. Details of solution methods and model parameters}

The purpose of this appendix is to give full details of the solution methods employed in the main text of the paper, as well as to provide the parameter values that are pertinent to obtaining solutions of the model. This appendix is structured as follows. Appendix D.1 specifies the fluid flow field within the drop. Appendix D.2 revisits the boundary layer theory, introducing a minor change to the governing equations, which has barely any effect upon the solutions themselves, but does make the solutions slightly easier to determine. Appendix D.3 explains details of identifying the time at which we switch from a boundary layer formulation to a streamline-averaged one. Appendix D.4 then sets up the streamline-averaged formulation, and Appendix D.5 describes how it is solved. An overall summary is given in Appendix D.6.

\section{Appendix D.1. Specification of fluid flow field}

In order to implement the theories outlined in section 2 , it is first necessary to specify the drop flow field via a streamfunction. We employ the same streamfunction as was used by Uribe-Ramirez and Korchinsky (2000b) and Ubal et al. (2010). This represents a truncated Galerkin expansion of the flow field produced with Reynolds number $R e=30$ assuming the same viscosity inside and outside the drop, and similar density inside and outside also (notwithstanding that a small density difference is ordinarily required to provide a buoyancy force to drive drop motion in the first place). The streamfunction is

$$
\psi=\left(e_{1} r^{2}+e_{2} r^{3}+e_{3} r^{4}\right) \sin ^{2} \theta+\left(e_{4} r^{2}+e_{5} r^{3}+e_{6} r^{4}\right) \sin ^{2} \theta \cos \theta
$$

where $e_{1}=0.390, e_{2}=-0.190, e_{3}=-0.200, e_{4}=0.012, e_{5}=0.288$ and $e_{6}=-0.300$.

Whilst the results of our model are sensitive to the streamfunction we use, the general procedure we follow is not. It is therefore possible to replace equation (D.1) by any other streamfunction, e.g. the Hadamard-Rybczynski streamfunction (Batchelor, 1967), that 
is applicable for a creeping flow, and repeat the same procedure in that case (Brignell, 1975; Kronig and Brink, 1950; Prakash and Sirignano, 1978). A comparison between the streamline layout for equation (D.1) and for Hadamard-Rybczynski streamfunction is presented in Ubal et al. (2010).

\section{Appendix D.1.1. Flow field at the drop surface}

The boundary layer theory of section 2.2 pays particular attention to the flow on the drop surface, and for the streamfunction given in equation (D.1) this becomes

$$
\left.u_{\theta}\right|_{\text {surf }}=\alpha \sin \theta+\beta \sin \theta \cos \theta
$$

where $\alpha=-2 e_{1}-3 e_{2}-4 e_{3}$ and $\beta=-2 e_{4}-3 e_{5}-4 e_{6}$. For the values of $e_{1}$ through $e_{6}$ that are considered here, we find $\alpha=0.590$ and $\beta=0.312$.

According to equations (D.1)-(D.2) there are stagnation points on the drop surface and axis, a forward stagnation point (at $r=1, \theta=0$ ) and a rear stagnation point (at $r=1, \theta=\pi)$. The forward stagnation point turns out to correspond to compression along the drop axis, i.e. biaxial extension normal to this, with extension rate $E_{\text {biax }}=$ $(\alpha+\beta)$. The rear stagnation point corresponds to uniaxial extension with extension rate $E_{\text {uniax }}=2(\alpha-\beta)$. Knowing the values of $E_{\text {biax }}$ and $E_{\text {uniax }}$ is important as these values affect the amount of time that fluid elements spend held up near each stagnation point: see Appendix A for more detail.

\section{Appendix D.1.2. Flow field near the internal stagnation point}

In addition to the forward and rear stagnation points, there is an additional internal stagnation point, about which all other streamlines circulate. This particular stagnation point corresponds to a local maximum of the streamfunction, which we denote by $\psi_{\text {stag }}$, and its location $\left(r_{\text {stag }}, \theta_{\text {stag }}\right)$ can be found by setting $u_{r}=0$ and $u_{\theta}=0$ in equations (4)-(5) respectively, and applying a Newton-Raphson technique (Press et al., 1992). We actually iterated the Newton-Raphson technique until it converged within round-off error of the computer used, but here we report results to just 3 significant figures, namely $r_{\text {stag }}=0.698$, $\theta_{\text {stag }}=1.38, \psi_{\text {stag }}=0.0810:$ again these values are sensitive to the $e_{1}$ through $e_{6}$ values 
employed in the particular flow field here. Any other flow field we might choose must however also have an internal stagnation point somewhere within the drop: that is a requirement of the topology of the streamline pattern. We also analysed the vorticity and the principal strain rates at the computed stagnation point ${ }^{10}$. The vorticity $\omega_{\text {stag }}$ turned out to be $\omega_{\text {stag }}=2.36$, and the principal strain rates were $\pm E_{\text {stag }}$ with $E_{\text {stag }}=0.628$.

Knowing the vorticity $\omega_{\text {stag }}$ is important since it affects one of the boundary conditions for the streamline-averaged theory (see equation (33) and also Appendix C). The values of $\omega_{\text {stag }}$ and $E_{\text {stag }}$ also turn out to affect the period of the streamline orbits immediately adjacent to the stagnation point. As is mentioned in Appendix C, these orbits are elliptical in shape. As one moves closer to the stagnation point, the length of the orbits shrink, but the velocity also falls, with the result that $T_{\text {orbit }}$ at the stagnation point approaches a well-defined limit, which we denote $T_{\text {orbit,stag, }}$, and which satisfies

$$
T_{\text {orbit,stag }}=\frac{4 \pi}{\omega_{\text {stag }}}\left(1-\frac{4 E_{\text {stag }}^{2}}{\omega_{\text {stag }}^{2}}\right)^{-1 / 2}
$$

We omit the proof of this result here, although we motivate the result by considering limiting cases as follows. For a fluid executing circular streamline orbits in solid body rotation with an angular frequency $\Omega$, it is simple to demonstrate that vorticity $\omega$ is $2 \Omega$, whereas rate of strain vanishes. The period of the solid body rotation is $2 \pi / \Omega$ and hence $4 \pi / \omega$ which agrees with what equation (D.3) predicts in the limit of vanishing strain rates. As strain rates grow for fixed vorticity, streamline orbits become increasingly elongated, and orbit times likewise increase according to equation (D.3): in the limit of a simple shear flow (for which $E_{\text {stag }} \rightarrow \omega_{\text {stag }} / 2$ ), equation (D.3) predicts orbit times will diverge.

The situation we are considering here has $0<E_{\text {stag }}<\omega_{\text {stag }} / 2$ and hence falls somewhere between the two limiting cases discussed above. For the $E_{\text {stag }}$ and $\omega_{\text {stag }}$ values considered here $\left(\omega_{\text {stag }}=2.36\right.$ and $E_{\text {stag }}=0.628$ as given above), we evaluate $T_{\text {orbit,stag }}$ as 6.28. This value is remarkably close to $2 \pi$, and hence has the same orbit time as a solid

\footnotetext{
${ }^{10}$ At the stagnation point, only two principal strain rates in the $(r, \theta)$ plane need to be considered, and they are opposite and equal $\pm E_{\text {stag. }}$. Away from a stagnation point however, azimuthal strain rates can occur, even though motion itself is confined to the $(r, \theta)$ plane: in such cases, principal strain rates in the $(r, \theta)$ plane cease to be opposite and equal.
} 
body rotation with unit angular frequency would have.

This completes our analysis of the main features of the flow field for the moment, although we will revisit the flow field later on in Appendix D.4 when we come to compute the parameters required by the streamline-averaged theory. Before considering that however, we discuss how to implement the boundary layer theory.

\section{Appendix D.2. Implementing the boundary layer theory}

The theory we have presented in section 2, including the boundary layer theory of section 2.2, assumes that convection is dominant (in the sense of having a large Peclet number). Near the forward and rear stagnation points, the situation is slightly more complicated since precisely at these stagnation points there is no flow at all and hence no convection: strictly speaking in the neighbourhood of such points adjustments to the boundary layer theory should be made.

We have already described (see section 2.2) how we handle the rear stagnation point, namely by specifying an angle $\theta_{\mathrm{r}}$ (close to $\pi$ ) at which flow is considered to return from the drop surface into the drop interior. Typically we choose $\theta_{\mathrm{r}}=\frac{99 \pi}{100}$, although we emphasise (again see section 2.2) that different choices of $\theta_{\mathrm{r}}$ only affect the balance between the amount of solute mass considered to be close to the drop surface, and the amount of mass considered to be already in the drop interior: changing $\theta_{\mathrm{r}}$ does not affect the total amount of solute mass determined to be in the drop, which is the quantity of main interest.

We now handle the forward stagnation point in a similar way, i.e. considering that fluid newly arriving from the drop interior (with zero solute mass fraction) was only injected into the boundary layer on the drop surface at a certain small but finite injection angle

$\theta_{\mathrm{i}}$. Typically we set $\theta_{\mathrm{i}}=\frac{\pi}{100}$ although we also considered choices of $\theta_{\mathrm{i}}=\frac{\pi}{10}$ or $\theta_{\mathrm{i}}=\frac{\pi}{1000}$. Introducing this parameter $\theta_{\mathrm{i}}$ to the boundary layer model affects the model's behaviour in a subtle way explained in detail below (Appendix D.2.1-Appendix D.2.3), the resulting solution scheme then being described in Appendix D.2.4-Appendix D.2.6. 
Appendix D.2.1. Role of the injection point $\theta_{\mathrm{i}}$

Since the boundary theory supposes that material points can only begin acquiring solute via diffusion once they arrive at the surface, i.e. once they arrive at angle $\theta_{\mathrm{i}}$, the "extent of diffusion" function $\zeta$ (introduced in section 2.2) is necessarily identically zero on the domain $0<\theta<\theta_{\mathrm{i}}$, and moreover for $\theta>\theta_{\mathrm{i}}$, equation (10) is replaced by

$$
\zeta=\left.\int_{\max \left(\theta_{\mathrm{i}}, \theta_{0}\right)}^{\theta} u_{\theta}\right|_{\operatorname{surf}} \sin ^{2} \theta \mathrm{d} \theta
$$

where recall from equation (9) that $\theta_{0}$ is the initial surface location of a fluid element currently at $\theta$ at time $T$.

If $\theta_{0}$ exceeds $\theta_{\mathrm{i}}$ then equation (D.4) is identical to equation (10). However if $\theta_{0}$ is less than $\theta_{\mathrm{i}}$ there is a difference. Because however we choose $\theta_{\mathrm{i}}$ to be a very small angle (i.e. $\left.\theta_{\mathrm{i}} \ll 1\right)$, the difference in question turns out to be exceedingly small. In the worst case, we consider a $\theta_{0}$ value that is arbitrarily small (tending to zero) and a small but finite $\theta_{\mathrm{i}}$. In the limit of small angles, equation (D.2) reduces to $\left.u_{\theta}\right|_{\text {surf }} \approx(\alpha+\beta) \theta$ and moreover $\sin \theta \approx \theta$, in which case the difference between equations (10) and (D.4), would become in this worst case $\int_{0}^{\theta_{\mathrm{i}}}(\alpha+\beta) \theta^{3} \mathrm{~d} \theta=(\alpha+\beta) \theta_{\mathrm{i}}^{4} / 4$. For $\theta_{\mathrm{i}}=\frac{\pi}{100}$, and for the $\alpha$ and $\beta$ values quoted earlier, the difference between equations (10) and (D.4) is then as little as $2 \times 10^{-7}$.

Appendix D.2.2. Points continuously on drop surface vs newly injected points

The change from equation (10) to equation (D.4) whilst minor in quantitative terms does actually imply a change in the way that the solutions for $\zeta$ behave. In order to understand the nature of this change, we first define a quantity $\theta_{\mathrm{b} \mid \mathrm{i}}$ which satisfies

$$
T=\int_{\theta_{\mathrm{i}}}^{\theta_{\mathrm{b} \mid \mathrm{i}}} \mathrm{d} \theta /\left.u_{\theta}\right|_{\text {surf }}
$$

This quantity $\theta_{\mathrm{b} \mid \mathrm{i}}$ (which clearly depends on $T$ ) corresponds to the trajectory followed by a fluid element which is injected onto the surface at location $\theta=\theta_{\mathrm{i}}$ when time $T=0$. Hence $\theta_{\mathrm{b} \mid \mathrm{i}}$ separates fluid elements which have continuously been in the boundary layer at the drop surface throughout the entire evolution of the system (namely those points with $\left.\theta>\theta_{\mathrm{b} \mid \mathrm{i}}\right)$ from other fluid elements which have been newly injected onto the surface after 
the evolution already started (namely those points with $\theta<\theta_{\mathrm{b} \mid \mathrm{i}}$ ). Observe that $\theta>\theta_{\mathrm{b} \mid \mathrm{i}}$ implies $\theta_{0}>\theta_{\mathrm{i}}$, whereas conversely $\theta<\theta_{\mathrm{b} \mid \mathrm{i}}$ implies $\theta_{0}<\theta_{\mathrm{i}}$.

Equation (D.4) implies that, for any given $\theta$, the value of $\zeta$ only evolves with time when $\theta>\theta_{\mathrm{b} \mid \mathrm{i}}$, since the time dependence of $\zeta$ (at any given $\theta$ ) manifests itself through the time evolution of the lower limit of integration in that equation. For $\theta<\theta_{\mathrm{b} \mid \mathrm{i}}$, the lower limit of integration in equation (D.4) remains fixed at $\theta_{\mathrm{i}}$, and hence the function $\zeta$ achieves a steady state, which does however still depend on $\theta$ : we denote this $\zeta_{\mathrm{ss}}(\theta)$, and discuss its implications in the next section.

\section{Appendix D.2.3. Steady state solution for extent of diffusion}

Knowing that a steady state solution $\zeta_{\mathrm{ss}}(\theta)$ will be attained, makes it slightly easier to compute solutions to equation (D.4) compared to equation (10), since one can stop the computations as soon as the steady state is achieved. Equation (10) by contrast does not attain a steady state at any finite time: for any chosen $\theta$, the lower integration limit of equation (10), namely $\theta_{0}$, continues decreasing with time even out to arbitrarily long times. Despite this subtle difference in behaviour, we reiterate that the difference between equations (10) and (D.4) remains small. In a worst case, we could have vanishingly small $\theta_{0}$ as a lower integration limit in equation (10) and the difference from equation (D.4) would be only $(\alpha+\beta) \theta_{\mathrm{i}}^{4} / 4$ which is negligibly small since we choose $\theta_{\mathrm{i}} \ll 1$.

\section{Appendix D.2.4. Solution scheme for $\theta_{\mathrm{b} \mid \mathrm{i}}$}

The boundary layer theory outlined in section 2.2 gives a solute mass fraction field expressed in terms of analytical formulae. However numerical computations remain necessary to obtain the values that are to be substituted into those analytical formulae. The first step in our numerical scheme was to compute $\theta_{\mathrm{b} \mid \mathrm{i}}$. In view of the known flow field on the drop surface (given by equation (D.2)), we found $\theta_{\mathrm{b} \mid \mathrm{i}}$ simply by solving the equation

$$
\mathrm{d} \theta_{\mathrm{b} \mid \mathrm{i}} / \mathrm{d} T=\left.u_{\theta}\right|_{\text {surf }}\left(\theta_{\mathrm{b} \mid \mathrm{i}}\right)=\alpha \sin \theta_{\mathrm{b} \mid \mathrm{i}}+\beta \sin \theta_{\mathrm{b} \mid \mathrm{i}} \cos \theta_{\mathrm{b} \mid \mathrm{i}}
$$

subject to an initial condition $\left.\theta_{\mathrm{b} \mid \mathrm{i}}\right|_{T=0}=\theta_{\mathrm{i}}$. This was achieved via a 4th order RungeKutta technique with a time step $\delta T=0.01$ and as mentioned previously we considered 
three different $\theta_{\mathrm{i}}$ values, $\frac{\pi}{1000}, \frac{\pi}{100}$ and $\frac{\pi}{10}$.

The level of truncation error for the Runge-Kutta scheme could be checked against an analytical solution: equation (D.6) admits an analytical solution in implicit form ( $T$ as a function of $\left.\theta_{\mathrm{b} \mid \mathrm{i}}\right)$. When we computed $\theta_{\mathrm{b} \mid \mathrm{i}}$ vs $T$ via the Runge-Kutta scheme, and then substituted the $\theta_{\mathrm{b} \mid \mathrm{i}}$ that was obtained back into the implicit formula, we recovered the original $T$ value through to 7 significant figures.

\section{Appendix D.2.5. Solution scheme for $\zeta$}

After determining $\theta_{\mathrm{b} \mid \mathrm{i}}$ vs $T$, we selected a set of times at which to examine the $\zeta$ vs $T$ profiles in detail. These times were $T=1, T=5$ and $T=10$. As a point of reference for $\theta_{\mathrm{i}}=\frac{\pi}{100}$ and $\theta_{\mathrm{r}}=\frac{99 \pi}{100}$, the value of $\theta_{\mathrm{b} \mid \mathrm{i}}$ began to exceed $\theta_{\mathrm{r}}$ at time 18.08 , the same time value quoted in sections 4.1, 4.2.6, 4.3.2 and 4.3.3: for times $T$ in excess of this, $\zeta$ has already achieved its final steady state value $\zeta_{\mathrm{ss}}(\theta)$ for these particular $\theta_{\mathrm{i}}$ and $\theta_{\mathrm{r}}$ values.

For each of the above mentioned times $T$, we divided the surface of the drop into 100 equispaced intervals between $\theta_{\mathrm{i}}$ and $\theta_{\mathrm{b} \mid \mathrm{i}}(T)$ as well as 100 equispaced intervals between $\theta_{\mathrm{b} \mid \mathrm{i}}(T)$ and $\theta_{\mathrm{r}}$. For each of the 100 equispaced $\theta$ values in the domain $\theta>\theta_{\mathrm{b} \mid \mathrm{i}}(T)$, we determined the corresponding $\theta_{0}$, by integrating the equation

$$
\mathrm{d} \theta / \mathrm{d} T=\left.u_{\theta}\right|_{\operatorname{surf}}(\theta)=\alpha \sin \theta+\beta \sin \theta \cos \theta
$$

backwards in time, starting from the selected $\theta$ value at time $T$ and continuing down to time zero. This was again achieved via a 4th order Runge-Kutta method analogous to the one described above, and gave the $\theta_{0}$ value corresponding to each selected $\theta$ and $T$.

Values of $\zeta$ could now be obtained via equation (D.4), integrating either from $\theta_{0}$ to $\theta$ (for each of the $\theta$ values greater than $\theta_{\mathrm{b} \mid \mathrm{i}}$ ), or integrating from $\theta_{\mathrm{i}}$ to $\theta$ (for each of the $\theta$ values less than $\left.\theta_{\mathrm{b} \mid \mathrm{i}}\right)$. For each of these integrations we used a Simpson's rule dividing the corresponding integration domain into 1000 equispaced intervals.

Appendix D.2.6. Solution scheme for solute masses $M_{\mathrm{surf}}, M_{\mathrm{ret}}$ and $M$

Once the $\zeta$ values were obtained as described above, it was necessary to compute the solute masses $M$ that have entered the drop (via summing equation (15) for solute mass 
located near the drop surface $M_{\text {surf }}$ and equation (17) for solute mass returned to the drop interior $M_{\text {ret }}$ ). The modifications to the solution introduced in Appendix D.2 imply that $\zeta$ is identically zero on the domain $0<\theta<\theta_{\mathrm{i}}$, and hence $M_{\text {surf }}$ becomes

$$
M_{\text {surf }}=2 \pi \int_{\theta_{\mathrm{i}}}^{\theta_{\mathrm{r}}} \sqrt{4 \zeta /(P e \pi)} \mathrm{d} \theta /\left.u_{\theta}\right|_{\text {surf }} .
$$

Since we have computed $\zeta$ values on 100 equispaced intervals both between $\theta_{\mathrm{i}}$ and $\theta_{\mathrm{b} \mid \mathrm{i}}$ and between $\theta_{\mathrm{b} \mid \mathrm{i}}$ and $\theta_{\mathrm{r}}$, the value of $M_{\text {surf }}$ in equation (D.8) can be computed via Simpson's rule. The mass returned to the interior of the drop, denoted $M_{\text {ret }}$ could be determined by applying Simpson's rule to equation (17), but integrating now over time, rather than over position. A time step $\delta T=0.02$ was used for the integration.

According to equations (17) and (D.8), the values of $M_{\text {surf }}$ and $M_{\text {ret }}$ (and hence also of $\left.M \equiv M_{\text {surf }}+M_{\text {ret }}\right)$ are sensitive to Peclet number. It is therefore necessary to specify a Peclet number in order to compute $M$. Remembering that we have stated that typical Peclet numbers for this process are on the order of tens of thousands, we chose $P e=10000$ as a "base case". The variation of $M_{\text {surf }}$ and $M_{\text {ret }}$ (and hence of $M$ ) with Peclet number is very straightforward: the mass transferred at any given $T$ value is proportional to $P e^{-1 / 2}$. Hence it is only necessary to compute $M$ vs $T$ for a single "base case" Peclet number, and values for any other Peclet number are obtained by rescaling, assuming that the Peclet number remains large enough that a boundary layer approximation (from which equations (17) and (D.8) are derived) applies. To ensure that this would be the case, we considered values from the "base case" $P e=10000$ upwards (up to $P e=100000$ ).

In addition to being sensitive to Peclet number, the boundary layer theory can also be sensitive to the flow field in the drop, and we have assumed a very particular flow field with a very particular streamline layout in Appendix D.1. Changing the flow field in the drop without changing the streamline topology would change the predictions of the boundary layer theory quantitatively, but not the general approach to computing the amount of solute transferred via equations (17) and (D.8).

A change in streamline topology (as can happen when the wake separates from the drop as Reynolds number increases (Juncu, 1999; Yan et al., 2002)) would require a modification 
to the approach to computing the solute mass transfer. The streamline pattern now consists of a main vortex filling most of the drop (Juncu, 1999; Yan et al., 2002) with a much weaker counter-rotating vortex in the neighbourhood of the rear of the drop. Whilst solute mass transfer in the weak counter-rotating vortex is expected to be dominated by diffusion, a convective boundary layer remains relevant to mass transfer in the main vortex. To compute the solute mass transfer in the main vortex, $\theta_{\mathrm{r}}$ in equations (17) and (D.8) must be placed adjacent to that separation point, not at the rear of the drop. In the interests of simplicity however we have ignored these more complicated streamline topologies, restricting consideration to cases that do not exhibit any wake separation, but that have instead a single vortex filling the entire volume of the drop.

This completes our discussion of how the solution of the boundary layer equations was implemented. The following subsection deals with determining the time at which the systems switches from a boundary layer formulation to a streamline-averaged one.

\section{Appendix D.3. Switching time from boundary layer to streamline-averaged formulation}

The solute mass $M$ that has entered the drop at any time $T$ (as determined above) can be related to the volume $V$ that this solute mass invades: in fact as was already mentioned in section 2.3.2 the solute concentrations have been normalised so as to ensure that $V=M$. As was discussed (see sections 4.3-4.4), apart from an initial transient, the numerical $V$ vs $T$ relation approximated very well to a straight line

$$
V \approx(a+b T) / \sqrt{P e}
$$

where $a$ and $b$ are empirical coefficients which turn out to be $a \approx 7.99$ and $b \approx 6.28$.

According to the theory in section 2.3, this must be matched to a relation for the volume $V$ enclosed by streamlines expressed as a function of the streamline orbit times $T_{\text {orbit }}$ : see equation (26). The parameters $A$ and $B$ appearing in equation (26) are inherited from the relation for $T_{\text {orbit }}$ vs $\psi$ (equation (23)) which ultimately can be related to the flow field, and hence in our particular case to the parameters $e_{1}$ through $e_{6}$ in equation (D.1).

The procedure for obtaining $A$ and $B$ is outlined in Appendix A and consists of analytic 
approximations to find hold up times near the forward and rear stagnation points, coupled to quadratures to compute traverse times along the remainder of the drop surface and axis. Specifically we employed analytic approximations near the forward stagnation point for $r>0.99$ and $\theta<\frac{\pi}{100}$, and likewise near the rear stagnation point for $r>0.99$ and $\pi-\theta<\frac{\pi}{100}$. Outside these domains, quadratures were performed (via Simpson's rule) using 4000 points on the drop surface and 2000 points on the drop axis. The end result was that $A=2.35$ and $B=-0.663$.

At first sight it is counterintuitive that $B$ is negative, since we expect the logarithmic term (which arises from hold up near the forward and rear stagnation points) to be added to the positive traverse times computed by quadratures. The negative $B$ value is however an artifact of the way the expression in equation (23) has been written. Remember (see Appendix D.1.2) that $\psi$ is never any larger than $\psi_{\text {stag }}$ and in fact near the drop surface and axis $\psi$ is much smaller than $\psi_{\text {stag. }}$. We can write equation $(23)$ in the form

$$
T_{\text {orbit }} \sim A \log \left(\psi_{\text {stag }} / \psi\right)+B^{\prime}
$$

where $B^{\prime}=A \log \left(1 / \psi_{\text {stag }}\right)+B$. Recalling from Appendix D.1.2 that $\psi_{\text {stag }}=0.0810$, and using the $A$ and $B$ values reported above, we find $B^{\prime}=5.24$. Equation (D.10) only applies for $\psi \ll \psi_{\text {stag }}$, meaning there is no need for $B^{\prime}$ to equal $\lim _{\psi \rightarrow \psi_{\text {stag }}} T_{\text {orbit }}$ which we already know from Appendix D.1.2 is $T_{\text {orbit,stag }} \approx 6.28$.

Having obtained $A$ and $B$ (or equivalently $A$ and $B^{\prime}$ ) we now have to seek a value of $V$ such that the $T$ value given by equation (D.9) intersects the $T_{\text {orbit }}$ value given by equation (26). The intersection point is the switching time $T_{\text {switch }}$ that we seek. That there is a unique intersection point (for any given $P e$ ) can be seen from the fact that equation (D.9) gives $V$ as an increasing function of $T$, whereas equation (26) gives $V$ as a decreasing function of $T_{\text {orbit }}$. In our computations, the approximate location of the intersection point was read off graphically (for any given $P e$ ) from plots of $V$ vs $T$ and $V$ vs $T_{\text {orbit }}$, subsequently Newton-Raphson iteration was used to find a simultaneous solution of equations (26) and (D.9) to within round-off error of the computer used. 
Appendix D.4. Setting up the streamline-averaged formulation

As was described in section 2.4, the streamline-averaged formulation leads to a diffusion equation in streamfunction space (equation (32)), which in turn involves some coefficients, i.e. orbit times $T_{\text {orbit }}$ and effective diffusivity $D_{\text {eff }}$ which depend on streamfunction $\psi$, and which are themselves defined in terms of integrals along streamlines (see equations (22) and (31)). Before we can solve equation (32) itself, first it is necessary to evaluate these coefficients via quadrature along streamlines. However even before we can do that, we must compute where each streamline is located in the drop: this is done in Appendix D.4.1, the technique for obtaining $T_{\text {orbit }}$ and $D_{\text {eff }}$ is described next in Appendix D.4.2, and then Appendix D.4.3 discusses the streamline-averaged initial conditions.

\section{Appendix D.4.1. Computing the locations of streamlines}

The way that we computed the location of the streamlines is as follows. Knowing that the domain of $\psi$ values in the drop was 0 through $\psi_{\text {stag }}$, we selected 1024 target $\psi$ values (denoted $\left.\psi_{\text {target }}\right)$ in increments of $\psi_{\text {stag }} / 1024$. For each value of $\psi_{\text {target }}$, we considered the formula for the streamfunction (given by equation (D.1)) along the direction $\theta=\theta_{\text {stag }}$. For this streamfunction, there are necessarily two values of $r$ at which $\psi=\psi_{\text {target }}$, an upper value $r_{\text {target,upper satisfying }} r_{\text {target,upper }}>r_{\text {stag }}$, and a lower value $r_{\text {target,lower satisfying }}$ $r_{\text {target,lower }}<r_{\text {stag }}$. For any given $\psi_{\text {target }}$, both $r_{\text {target,upper }}$ and $r_{\text {target,lower }}$ were found via the Newton-Raphson technique. For $\psi \ll \psi_{\text {stag }}, r_{\text {target,upper }}$ turns out to be close to the drop surface, whereas $r_{\text {target,lower }}$ is close to the drop centre. However as $\psi$ increases towards $\psi_{\text {stag }}$, both $r_{\text {target,upper }}$ and $r_{\text {target,lower migrate towards }} r_{\text {stag }}$.

Nominally the points $\left(r_{\text {target,upper }}, \theta_{\text {stag }}\right)$ and $\left(r_{\text {target,lower }}, \theta_{\text {stag }}\right)$ that we compute should be on the same streamline. Since however the values of $r_{\text {target,upper }}, r_{\text {target,lower }}$ and $\theta_{\text {stag }}$ were only recorded to a finite precision (typically to 6 decimal places), when we substituted those values back into equation (D.1) and evaluated $\psi$ at both $\left(r_{\text {target,upper }}, \theta_{\text {stag }}\right)$ and $\left(r_{\text {target,lower }}, \theta_{\text {stag }}\right)$, a very slight difference in the resulting $\psi$ values was obtained, typically in the 6th significant figure. This tiny difference is however insignificant taking into account that with 1024 streamlines being computed, the relative difference in $\psi$ between one streamline and an adjacent one tends to be approximately 3 orders of magnitude 
larger than that, i.e. in the 3rd significant figure.

The points $\left(r_{\text {target,upper }}, \theta_{\text {stag }}\right)$ and $\left(r_{\text {target,lower }}, \theta_{\text {stag }}\right)$ are just two isolated points on a streamline, and the remaining points on that streamline were obtained by integrating

$$
\begin{aligned}
& \mathrm{d} r / \mathrm{d} t=u_{r} \\
& \mathrm{~d} \theta / \mathrm{d} t=u_{\theta} / r
\end{aligned}
$$

where $u_{r}$ and $u_{\theta}$ are given by equations (4)-(5). This integration was done via Heun's method with a time step of 0.001 and subsequently repeated with a time step of $5 \times 10^{-4}$ to check sensitivity to time step.

Equations (D.11)-(D.12) correspond to following streamlines, like those sketched in Figure 1(a), in a clockwise sense. If we follow a streamline starting from $\left(r_{\text {target,upper }}, \theta_{\text {stag }}\right)$, the $\theta$ value on the streamline increases above $\theta_{\text {stag }}$ and then starts to decrease, falling back towards $\theta_{\text {stag }}$ once the streamline reaches the neighbourhood of $\left(r_{\text {target,lower }}, \theta_{\text {stag }}\right)$. As soon as $\theta$ falls below $\theta_{\text {stag }}$, we stop the Heun's method integration, interpolating the final integration interval to retain data only as far as $\theta_{\text {stag. }}$. The resulting $r$ value should

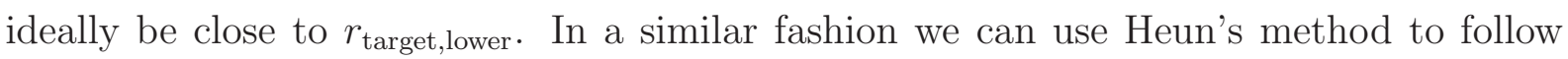
the streamline starting from $\left(r_{\text {target,lower }}, \theta_{\text {stag }}\right)$. As before the streamline is being followed in a clockwise fashion, but now the value of $\theta$ should initially decrease, and then increase

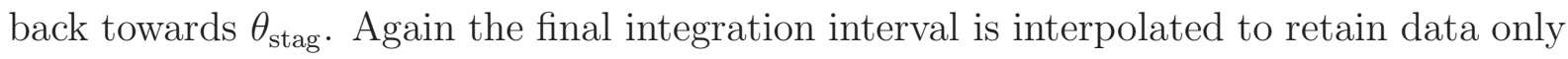
up to $\theta_{\text {stag }}$, and the final $r$ value should be in the neighbourhood of $r_{\text {target,upper }}$.

Heun's method involves a truncation error, which is equivalent to saying that the numerical points that we compute gradually drift away from the streamline upon which they should be placed. We quantify this truncation error by computing the $\psi$ values at the end point of each integration, comparing them with the $\psi$ values at the starting point. For integration time step 0.001 and $5 \times 10^{-4}$, this deviation in $\psi$ between start and end points was respectively $13 \%$ and $3 \%$ of the difference in $\psi$ between $\left(r_{\text {target,upper }}, \theta_{\text {stag }}\right)$ and $\left(r_{\text {target,lower }}, \theta_{\text {stag }}\right):$ remember that the latter difference was itself very tiny (in the 6 th significant figure) and arose due to $r_{\text {target,upper }}, r_{\text {target,lower }}$ and $\theta_{\text {stag }}$ only being recorded to finite 6 decimal place precision. In effect this means that it is unnecessary to use 
smaller time steps than those we have already used in our Heun method integration: the error accumulated during that integration along the streamline is already less than that involved in identifying the locations of $r_{\text {target,upper }}, r_{\text {target,lower }}$ and $\theta_{\text {stag }}$ in the first place.

\section{Appendix D.4.2. Computing streamline orbit time and effective diffusivity}

Having computed the locus swept out by each of 1024 streamlines, we can now proceed to compute $T_{\text {orbit }}$ and $D_{\text {eff }}$ via equations (22) and (31). This is done via quadrature (using the trapezoidal rule), using the streamline loci we have already determined.

Note in particular that $T_{\text {orbit }}$ in general does not follow equation (23) since that equation only applies to $\psi \ll \psi_{\text {stag }}$, whereas here we consider the full range of $\psi$ values up to

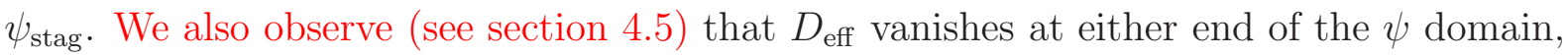
both as $\psi \rightarrow 0$ and $\psi \rightarrow \psi_{\text {stag. }}$. It follows that somewhere, for one of the streamlines with $0<\psi<\psi_{\text {stag }}$, $D_{\text {eff }}$ must attain a maximum. We denote this local maximum by $D_{\text {eff,max }}$, and its value turns out to be 0.0678 (to 3 figures) and moreover it corresponds to streamline number 192 out of the 1024 that we calculated (counting streamlines from the drop surface/axis towards the internal stagnation point). Determining the value of $D_{\text {eff,max }}$, turns out to be relevant to formulating the numerical algorithm for solving the streamline-averaged equation (32). We will explain more about this in Appendix D.5.

It is worth recalling that we obtained our formulae for $T_{\text {orbit }}$ and $D_{\text {eff }}$ (and hence also $\left.D_{\text {eff,max }}\right)$ for a very particular flow field given in Appendix D.1. Were we to change the flow field inside the drop, the values of $T_{\text {orbit }}$ and $D_{\text {eff }}$ would be sensitive to how the streamlines are laid out in space and also to the speed of the internal flow. If, for a fixed streamline pattern, we changed the speed of the flow inside the drop, multiplying all flow speeds $u_{s}$ by some uniform factor ${ }^{11} f_{u}$, equations (22) and (31) indicate that $T_{\text {orbit }}$ would scale inversely with $f_{u}$, whereas $D_{\text {eff }}$ would scale proportional to the square of $f_{u}$, whilst $\psi$ and $\psi_{\text {stag }}$ would be proportional to $f_{u}$. The time evolution of the mass fraction in the drop given by equation (32) over the solution domain $0 \leq \psi \leq \psi_{\text {stag }}$ would however remain unaffected. We

\footnotetext{
${ }^{11}$ The factor $f_{u}$ here refers not to changing the drop speed (since that is determined by Peclet number), but rather to changing the speed of the internal drop circulation relative to the drop speed. Such a change could be achieved by manipulating the viscosity ratio between internal and external liquids.
} 
conclude that despite $T_{\text {orbit }}$ and $D_{\text {eff }}$ being sensitive to flow speed, the streamline-averaged theory itself is not sensitive to that, depending on the flow field only to the extent that the spatial layout of the streamlines is affected by that field. We selected a very particular flow field in Appendix D.1 but the spatial layout of the streamlines is qualitatively similar (Ubal et al., 2010) to that of other flow fields e.g. Hadamard-Rybczynski flow. Hence the results we present for the streamline-averaged theory should likewise be qualitatively similar to what is obtained for a Hadamard-Rybczynski flow (Brignell, 1975; Kronig and Brink, 1950; Prakash and Sirignano, 1978).

\section{Appendix D.4.3. Determining initial condition for the streamline-averaged formulation}

A challenge associated with setting up the streamline-averaged formulation is identifying a suitable distribution for the solute mass within the drop to use as an initial condition within that formulation. Mass must be redistributed from a boundary layer mass fraction field (equation (11)), which envisages non-uniform mass fraction along streamlines with the mass fraction being larger wherever the extent of diffusion $\zeta$ is larger, to a streamlineaveraged field (equation (34)) with uniform mass fraction along streamlines.

Since we have already identified the switching time at which this is to happen (see Appendix D.3), and hence the corresponding total solute mass $M$ in the drop at this time, our task is to integrate the right hand side of equation (35) by quadrature and, by adjusting the parameter $\psi_{*}$ within this equation, match it to the known $M$ value on the left hand side. We know that the maximum value of $\psi$ in the drop is $\psi_{\text {stag }}$, and we can shift the upper limit of the integral in equation $(35)$ to the value $\psi_{\text {stag }}$, which makes negligible error since we anticipate $\psi_{*} \ll \psi_{\text {stag }}$, implying that the integrand is

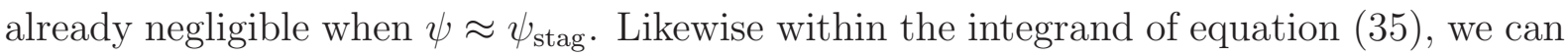
approximate $T_{\text {orbit }}$ by equation (23), which applies for $\psi \ll \psi_{\text {stag }}$. Performing quadrature on equation (35) remains complicated by $T_{\text {orbit }}$ diverging as $\psi \rightarrow 0$, although the function remains integrable. For any chosen $\psi_{*}$ value, we integrated analytically over the domain $0 \leq \psi \leq 0.001 \psi_{*}$ approximating $W=\operatorname{erfc}\left(\psi / \psi_{*}\right)$ from equation $(34)$ by $W \approx 1-\frac{2}{\sqrt{\pi}} \psi / \psi_{*}$ in this domain. Over the rest of the integration domain we used an adaptive quadrature routine (quad_qags) built into the computer algebra package "maxima". Having thereby 
computed the right hand side of equation (35) for any chosen $\psi_{*}$, we recomputed it for values of $\psi_{*}$ that were larger or smaller by $0.1 \%$. This permitted us to compute numerically the derivative of the right hand side of equation (35) with respect to $\psi_{*}$, and apply Newton-Raphson iteration to find the target value of $\psi_{*}$ satisfying equation (35).

The value of $\psi_{*}$ that we obtained was sensitive to Peclet number, since the value of $M$ at which we begin to use the streamline-averaged theory is also sensitive to Peclet number: higher Peclet numbers imply switching at smaller $M$ values and hence smaller $\psi_{*}$, as was already anticipated in section 2.5.1. On the other hand, according to section 2.3.2, higher Peclet number also implies switching to the streamline-averaged state at earlier times (at least when time is measured on a diffusive scale), so higher Peclet number systems spend more time in the streamline-averaged state, thereby compensating for having entered that state with less solute. Moreover the governing equation (32) for the streamline-averaged theory has no Peclet number dependence whatsoever, so any slight sensitivity that the initial condition might exhibit to $P e$ is rapidly lost as the system evolves over time.

This completes the setting up of the streamline-averaged formulation. The solution technique used to solve the streamline-averaged equations is described in the next section.

\section{Appendix D.5. Solving the streamline-averaged equations}

We solved the streamline-averaged equations using a finite difference Crank-Nicolson method (Press et al., 1992). We used $2^{N}$ intervals in streamfunction space, where $N$ is an integer that we select. Since we have already computed $T_{\text {orbit }}$ and $D_{\text {eff }}$ values for 1024 streamlines in total, we could choose $N$ up to 10 . Choosing smaller values of $N$ corresponds to using a coarser grid: e.g. $N=9$ retains every 2 nd streamline out of the original 1024 that we computed, $N=8$ retains every 4 th streamline, etc..

The increment in $\psi$ from streamline to streamline that we denote $\delta \psi$ now equals $\psi_{\text {stag }} / 2^{N}$. The time step $\delta t$ for the Crank-Nicolson method was chosen such that $\delta t=$ $\delta \psi^{2} /\left(4 D_{\text {eff,max }}\right)$, which is sufficient to ensure stability of the Crank-Nicolson scheme. Clearly determining $D_{\text {eff,max }}$ in advance as we have done in Appendix D.4.2 is important because $D_{\text {eff,max }}$ affects the time step for the numerical scheme.

Streamlines are labelled from $i=0$ to $i=2^{N}$ with $i=0$ giving the drop surface and 
axis, and $i=2^{N}$ giving the internal stagnation point. Finite differencing equation (32) in space gives us the rate of change of solids fraction $W$ on streamline $i$ for all $i$ values in the domain $1 \leq i \leq 2^{N}-1$ in terms of the values of $W$ (on streamline $i$ and also on the nearest neighbour streamlines $i \pm 1$ ), as well as in terms of the values of $D_{\text {eff }} T_{\text {orbit }}$ (again on streamline $i$ and the nearest neighbour streamlines $i \pm 1$ ), and the value of streamline orbit time $T_{\text {orbit }}$ (on streamline $i$, but importantly not on streamlines $i \pm 1$ ).

The fact that $T_{\text {orbit }}$ is required only on streamline $i$ but not on its neighbours, means in the case $i=1$ that the finite difference neatly avoids the singular behaviour of $T_{\text {orbit }}$ in the limit as $\psi \rightarrow 0$, the product $D_{\text {eff }} T_{\text {orbit }}$ being non-singular in this limit. Of course for $i=0$ a boundary condition applies $W=1$ for all times. At the other boundary, for which $\psi \rightarrow \psi_{\text {stag }}$ and hence $i=2^{N}$, the rate of evolution of $W$ is given by equation (33). We approximate this equation via finite difference, although the spatial derivative at this boundary is necessarily one-sided involving streamline $i=2^{N}$ and $i=2^{N}-1$, rather than a central difference which is used for all other streamlines in a Crank-Nicolson scheme.

Having now specified the spatial finite difference representation of the governing differential equation and of the boundary conditions, we can proceed to solve the equations using finite differences in time, starting from the initial condition given by equation (34): this is discussed further in Appendix D.5.1 below.

\section{Appendix D.5.1. Choosing the finite difference step size for Crank-Nicolson}

We implemented the Crank-Nicolson algorithm via a computer program in C.

The run time of the program is very sensitive to the parameter $N$, there being $2^{N}$ streamlines, and hence order $2^{N}$ operations per time step. However according to the formula given earlier for $\delta t$, the size of the time step scales like $1 / 2^{2 N}$, hence the computations required to reach any target final time grow like $2^{3 N}$. To achieve good computational speed we need to choose $N$ as small as possible, but without compromising computational accuracy. We performed calculations with different values of $N$, including $N=5$ (corresponding to 32 streamlines), $N=6$ (corresponding to 64 streamlines), and $N=7$ (corresponding to 128 streamlines).

For the "base case" $P e$ value we consider here $(P e=10000)$ we compared for different 
$N$ values the predicted time evolution of $W$ at the internal stagnation point. We only found discrepancies between the predictions for $N=6$ (64 streamlines) and those for higher values of $N$ only in the 4th (or higher) significant figure. Hence we adopted this value $N=6$ in all our subsequent calculations.

\section{Appendix D.6. Summary of solution methods and model parameters}

We summarise the discussion of Appendix D.1-Appendix D.5 as follows.

The model that we have solved here comprises an early-time boundary layer theory combined with a streamline-averaged theory at later time. The model predictions depend upon the flow field assumed and potentially also upon the Peclet number chosen. We employed a truncated Galerkin expansion for the flow field formerly used by Uribe-Ramirez and Korchinsky (2000b) and Ubal et al. (2010), although other choices are available (e.g. a Hadamard-Rybczynski flow, as mentioned in Appendix D.1). We chose a base case Peclet number of $P e=10000$ (a value which is typical for liquid-liquid extraction applications, see sections 1 and 2.1). However (see Appendix D.2.6) we also considered larger Peclet numbers up to $P e=100000$ to investigate the sensitivity to this parameter.

Despite the fact that it is necessary to choose such parameter values in order to compute with the model, the predictions that result can be rather insensitive to the choices made. This is particularly true of the streamline-averaged theory (see Appendix D.4-Appendix D.5), which is actually sensitive to how the streamlines are laid out in space rather than to the overall speed of the flow field. Moreover the Peclet number does not appear as an explicit parameter anywhere within the governing equations of the streamline-averaged theory, and merely affects the time at which we switch to computing with this particular theory and the initial condition applied at this time (specifically the parameter $\psi_{*}$ in equation (34) depends on Peclet number).

Computing with the model required us to implement a number of numerical techniques but the techniques are all fairly standard ones.

To implement the boundary layer theory (see Appendix D.2) we first needed to compute a quantity $\theta_{0}$ which is an angular location on the drop surface representing the initial location of a material point that reaches location $\theta$ by time $T$. In addition to computing 
$\theta_{0}$, it was necessary to select the value of a parameter $\theta_{\mathrm{i}}$ (the so called "injection point") which needs to be close to the forward stagnation point of the drop, and which we chose arbitrarily to be $\frac{\pi}{100}$ (although other choices e.g. $\frac{\pi}{10}$ or $\frac{\pi}{1000}$ are also possible). Despite this apparent arbitrariness in the choice of $\theta_{\mathrm{i}}$ there was no arbitrariness in the solute mass fraction field which resulted: this could be expressed (see equation (11)) in terms of an "extent of diffusion" function $\zeta$. For any given time $T$ and any given angular location $\theta$, this function $\zeta$ could be computed via quadrature (see equation (D.4)), the lower limit of the quadrature interval being $\max \left(\theta_{\mathrm{i}}, \theta_{0}\right)$ and hence depending upon whether $\theta_{0}$ is greater or less than $\theta_{\mathrm{i}}$. However, for situations in which a change in the value of $\theta_{\mathrm{i}}$ did indeed alter the lower bound of the interval, the value of the integrand in the neighbourhood of the lower bound was very small. Hence small shifts in $\theta_{\mathrm{i}}$ do not materially affect $\zeta$. Moreover once $\zeta$ was determined, the amount of mass $M$ entering the drop as a function of time $T$ could be obtained via quadrature (see Appendix D.2.6).

This mass $M$ was then related to a volume $V$ that this mass would fill, were it to be distributed at a specified concentration throughout those streamlines closest to the drop surface and axis (see section 2.3 and Appendix D.3). Thus a relation between volume $V$ filled by solute and elapsed time $T$ was established.

Streamline orbit times $T_{\text {orbit }}$ for streamlines close to the drop surface and axis were also computed, a relation being found between $T_{\text {orbit }}$ and the volume $V$ that these streamlines enclosed. Since the flow is held up for arbitrarily long times near the forward and rear stagnation points of the drop, computing $T_{\text {orbit }}$ required analytic approximations near these stagnation points, and quadrature outside these neighbourhoods.

The boundary layer theory was switched to a streamline-averaged theory once the solute that had entered the drop was deemed to have executed one full streamline circulation: this required that the time elapsed $T$ matched the streamline orbit time $T_{\text {orbit }}$ corresponding to the particular volume $V$ within the drop which the solute was deemed to fill. The switching time that satisfied $T=T_{\text {orbit }}$ along with the corresponding volume $V$ filled by solute (and hence the associated solute mass $M$ ) were determined by a Newton-Raphson technique. The switch between the boundary layer theory and the 
streamline-averaged theory was thereby taken to occur at a well-defined time and a welldefined amount of solute transferred, these values being dependent however upon the flow field assumed and upon the Peclet number.

The streamline-averaged equation was solved by a Crank-Nicolson finite difference technique. Coefficients in the streamline-averaged model $T_{\text {orbit }}$ and $D_{\text {eff }}$ for a selection of streamlines throughout the entire drop were computed by quadrature along streamlines, the locations of the streamlines themselves having first been obtained via Heun's method (see Appendix D.4). The initial condition for the streamline-averaged model assumed that the solute mass $M$ in the drop could be redistributed across streamlines according to a complementary error function (see equation (34)): the distribution involved a parameter $\psi_{*}$ which was fixed via a Newton-Raphson technique.

As is seen from the summary above, the numerical techniques employed were all standard ones, i.e. quadratures, Runge-Kutta, Heun's method, Newton-Raphson and CrankNicolson. Any complexity arises not from the numerical techniques themselves but rather from the fact that implementing the model requires assembling all the various techniques together. Results from the implementation of the model are described in the main text.

\section{References}

Abramzon, B., Borde, I., 1980. Conjugate unsteady heat-transfer from a droplet in creeping flow. AIChE J. 26.

Batchelor, G. K., 1967. An Introduction to Fluid Dynamics, first edition Edition. Cambridge University Press, Cambridge.

Brignell, A. S., 1975. Solute extraction from an internally circulating spherical liquid drop. Int. J. Heat Mass Trans. 18, 61-68.

Brodkorb, M. J., Bosse, D., von Reden, C., Gorak, A., Slater, M. J., 2003. Single drop mass transfer in ternary and quaternary liquid-liquid extraction systems. Chem. Eng. Process. 42, 825-840. 
Edelmann, C. A., Le Clercq, P. C., Noll, B., 2017. Numerical investigation of different modes of internal circulation in spherical drops: Fluid dynamics and mass/heat transfer. Int. J. Multiphase Flow 95, 54-70.

Handlos, A. E., Baron, T., 1957. Mass and heat transfer from drops in liquid-liquid extraction. AIChE J. 3, 127-136.

Johns, L. E., Beckmann, R. B., 1966. Mechanism of dispersed-phase mass transfer in viscous, single-drop extraction systems. AIChE J. 12, 10-16.

Juncu, G., 1999. A numerical study of steady viscous flow past a fluid sphere. Int. J. Heat Fluid Flow 20.

Juncu, G., 2005. Unsteady ternary mass transfer from a sphere in creeping flow. Int. J. Therm. Sci. 44, 255-266.

Juncu, G., 2011. Unsteady-state mass transfer from a binary gas bubble with changing volume. Int. J. Heat Mass Transfer 54, 669-677.

Jyothi, R. K., Lee, J. Y., Kim, J. S., Sohn, J. S., 2009. Liquid-liquid extraction of platinum from acidic solutions: A review. Solv. Extr. Res. Dev. 16, 13-22.

Korchinsky, W. J., Grassia, P., Harrison, C. H., 2009. Multicomponent mass transfer in films and rigid drops: the influence of concentration-variable diffusivity. Chem. Engng Sci. $64,433-442$.

Kronig, R., Brink, J. C., 1950. The theory of extraction from falling droplets. Appl. Sci. Res. A2, 142-154.

Kumar, A., Hartland, S., 1999. Correlations for prediction of mass transfer coefficients in single drop systems and liquid-liquid extraction columns. Chem. Eng. Res. Des. 77, $372-384$.

Leal, L. G., 2007. Advanced Transport Phenomena: Fluid Mechanics and Convective Transport Processes. Cambridge Series in Chemical Engineering. Cambridge University Press, Cambridge. 
Levich, V. G., Krylov, V. S., Vorotilin, V. P., 1965. A contribution to theory of unsteady diffusion from a moving drop. Dokl. Akad. Nauk. SSSR 161, 648-651.

Mazzola, P. G., Lopes, A. M., Hasmann, F. A., Jozala, A. F., Penna, T. C. V., Magalhaes, P. O., Rangel-Yagui, C. O., Pessoa, A., 2008. Liquid-liquid extraction of biomolecules: An overview and update of the main techniques. J. Chem. Technol. Biotechnol. 83, $143-157$.

Mohanty, S., 2000. Modeling of liquid-liquid extraction column: A review. Rev. Chem. Eng. 16, 199-248.

Moreno-Gonzalez, D., Garcia-Campana, A. M., 2017. Salting-out assisted liquid-liquid extraction coupled to ultra-high performance liquid chromatography-tandem mass spectrometry for the determination of tetracycline residues in infant foods. Food Chem. $221,1763-1769$.

Negri, E. D., Korchinsky, W. J., 1986. Multicomponent mass transfer in spherical rigid drops. Chem. Engng Sci. 41, 2395-2400.

Negri, E. D., Young, C. H., Korchinsky, W. J., 1986. High flux, single solute mass transfer in spherical rigid drops. Chem. Engng Sci. 41, 2401-2406.

Nishihama, S., Hirai, T., Komasawa, I., 2001. Review of advanced liquid-liquid extraction systems for the separation of metal ions by a combination of conversion of the metal species with chemical reaction. Ind. Eng. Chem. Res. 40, 3085-3091.

Oliver, D. L. R., Carleson, T. E., Chung, J. N., 1985. Transient heat transfer to a fluid sphere suspended in an electric field. Int. J. Heat Mass Transfer 28, 1005-1009.

Piarah, W. H., Paschedag, A., Kraume, M., 2001. Numerical simulation of mass transfer between a single drop and an ambient flow. AIChE J. 47 (7), 1701-1704.

Prakash, S., Sirignano, W. A., 1978. Liquid fuel droplet heating with internal circulation. Int. J. Heat Mass Trans. 21, 885-895. 
Press, W. H., Teukolsky, S. A., Vetterling, W. T., Flannery, B. P., 1992. Numerical Recipes in C. The Art of Scientific Computing, 2nd Edition. Cambridge University Press, Cambridge.

Richardson, J. F., Harker, J. H., Backhurst, J. R., 2002. Chemical Engineering, 5th Edition. Vol. 2: Particle Technology and Separation Processes. Butterworth-Heinemann, Oxford, Ch. 13: Liquid-Liquid Extraction, pp. 721-770.

Ruckenstein, E., 1967. Mass transfer between a single drop and a continuous phase. International Journal of Heat and Mass Transfer 10, 1785-1792.

Sadhal, S. S., Ayyaswamy, P. S., Chung, J. N., 1997. Transport Phenomena with Drops and Bubbles. Springer, New York, London.

Silva, M. E., Franco, T. T., 2000. Liquid-liquid extraction of biomolecules in downstream processing: A review paper. Braz. J. Chem. Eng. 17, 1-17.

Sirignano, W. A., 2010. Fluid Dynamics and Transport of Droplets and Sprays, 2nd Edition. Cambridge University Press, Cambridge.

Ubal, S., Grassia, P., Harrison, C. H., Korchinsky, W. J., 2011. Numerical simulation of multi-component mass transfer in rigid or circulating drops: Multi-component effects even in the presence of weak coupling. Colloids and Surf. A, Physicochem. and Engg Aspects 380, 6-15.

Ubal, S., Harrison, C. H., Grassia, P., Korchinsky, W. J., 2010. Numerical simulation of mass transfer in circulating drops. Chem. Engng Sci. 65, 2934-2956.

Uribe-Ramirez, A. R., Korchinsky, W. J., 2000a. Fundamental theory for prediction of multicomponent mass transfer in single-liquid drops at intermediate Reynolds numbers $(10<R e<250)$. Chem. Engng Sci. 55, 3319-3328.

Uribe-Ramirez, A. R., Korchinsky, W. J., 2000b. Fundamental theory for prediction of single-component mass transfer in liquid drops at intermediate Reynolds numbers $(10<$ $R e<250)$. Chem. Engng Sci. 55, 3305-3318. 
Vorotilin, V. P., Krylov, V. S., Levich, V. G., 1965. On the theory of extraction from a falling droplet. J. Appl. Math. Mech. 29, 386-394.

Waheed, M. A., Henschke, M., Pfennig, A., 2002. Mass transfer by free and forced convection from single spherical liquid drops. Int. J. Heat Mass Transfer 45, 4507-4514.

Wegener, M., Paul, N., Kraume, M., 2014. Fluid dynamics and mass transfer at single droplets in liquid/liquid systems. Int. J. Heat Mass Transfer 71, 475-495.

Yahaya, G. O., Hamad, F., Bahamdan, A., Tammana, V. V. R., Hamad, E. Z., 2013. Supported ionic liquid membrane and liquid-liquid extraction using membrane for removal of sulfur compounds from diesel/crude oil. Fuel Process. Technol. 113, 123-129.

Yan, Y., Lai, H., Gentle, C. R., Smith, J. M., 2002. Numerical analysis of fluid flows inside and around a liquid drop using an incorporation of multi-block iteration and moving mesh. Chem. Eng. Res. Des. 80, 325-331. 


\begin{tabular}{r|rrr|r}
$P e$ & $T_{\text {switch }}$ & $V$ & $\psi$ & $\psi_{*}$ \\
\hline 10000 & 10.37 & 0.7326 & 0.009157 & 0.0173 \\
20000 & 11.18 & 0.5537 & 0.006511 & 0.0122 \\
50000 & 12.24 & 0.3801 & 0.004144 & 0.00777 \\
100000 & 13.05 & 0.2848 & 0.002942 & 0.00549
\end{tabular}

Table 1: As a function of Peclet number $P e$, the switching time $T_{\text {switch }}$ at which the boundary layer theory is switched for a streamline-averaged one, the characteristic volume $V$ of the drop which has been invaded by solute at this time (determining $V$ as if the solute invades with the same concentration as it has outside the drop, and remembering also that the full drop volume is $\frac{4}{3} \pi \approx 4.188$ ), and the streamline $\psi$ enclosing this invaded volume (remembering that the internal stagnation point is $\psi_{\text {stag }} \approx 0.0810$ ). The final column gives the parameter $\psi_{*}$ that appears in the complementary error function (equation (34)) which governs the assumed distribution of solute in the drop when the streamline-averaged state is initiated (ensuring that the correct amount of solute is inherited from the earlier boundary layer regime). 
(a)

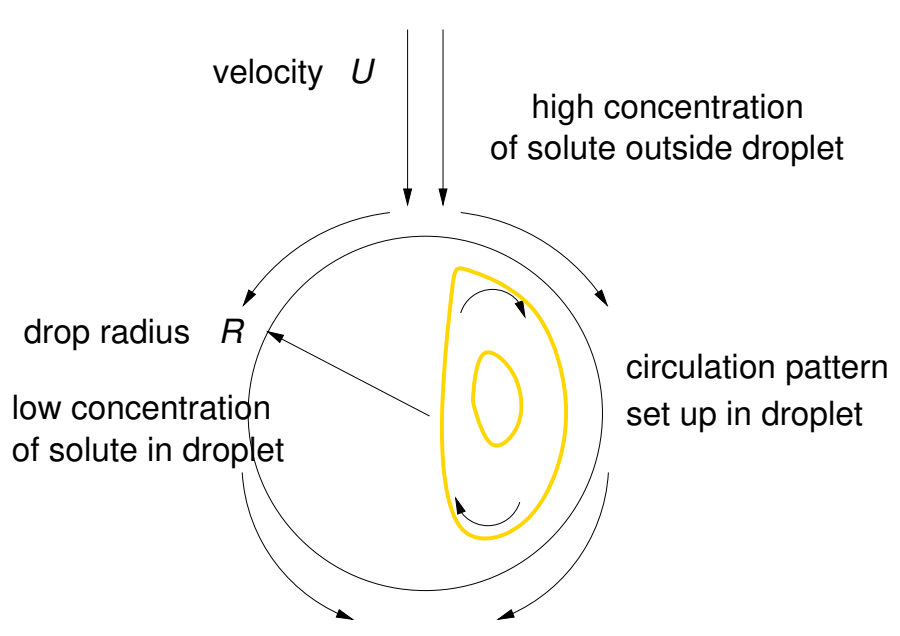

(b)

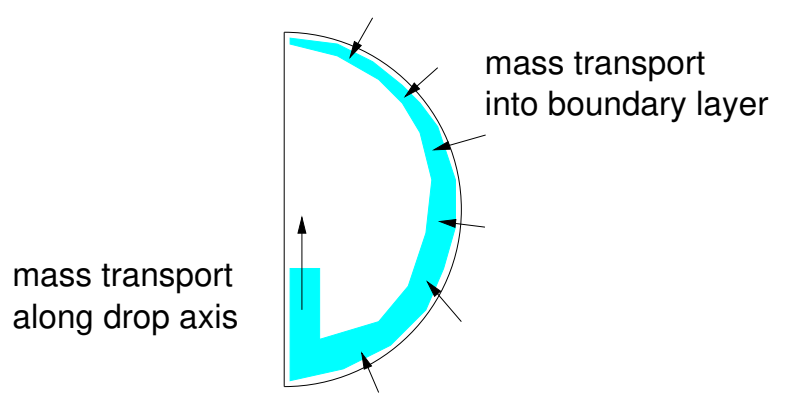

(c)

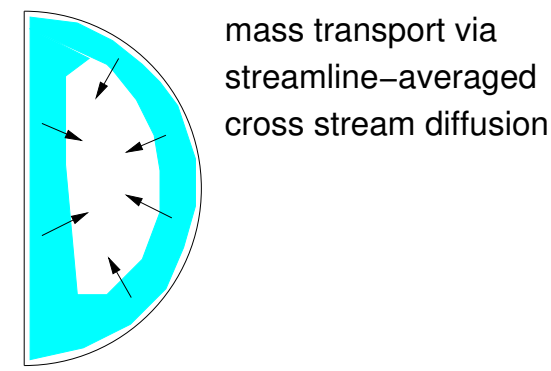

Figure 1: (a) A drop of radius $R$ is moving relative to surrounding liquid at speed $U$, which also sets up a circulation pattern inside the drop. Initially there is a high concentration of solute outside the drop and a low concentration of solute inside, such that mass is transported into the drop. (b) A boundary layer picture of the mass transport in which the mass transfer takes place primarily across a near surface boundary layer (shown shaded) but also with the streamline pattern causing solute to be transported into the drop interior part way up the drop axis (again shown shaded). (c) A streamline-averaged picture of mass transport in which solute concentration is uniform along streamlines, but mass is transferred in the cross-stream direction from a high concentration region near the drop surface and axis (shown shaded) to a low concentration region away from the surface and axis. 


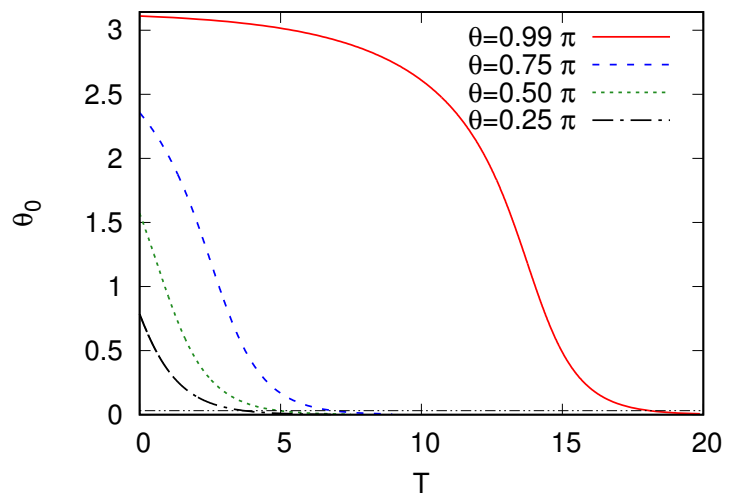

Figure 2: The angle $\theta_{0}$ as a function of $T$ for various $\theta$ values: $\theta_{0}$ is the initial location of the material point which happens to be at location $\theta$ at time $T$. The dash-dot line along the bottom of the figure corresponds to a set value of $\theta_{\mathrm{i}}$, chosen here to be $\frac{\pi}{100}$. 
(a)

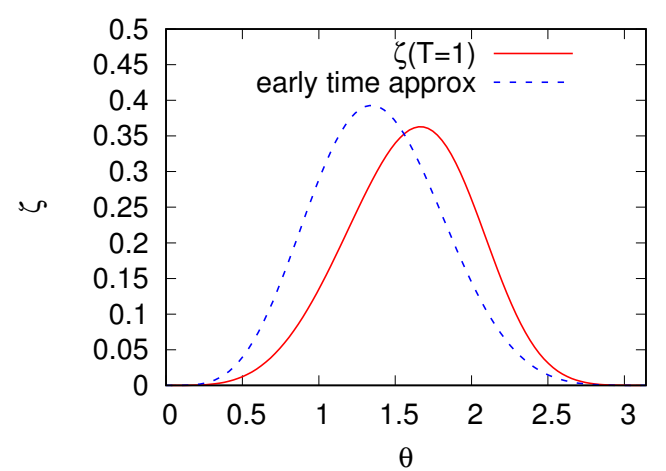

(b)

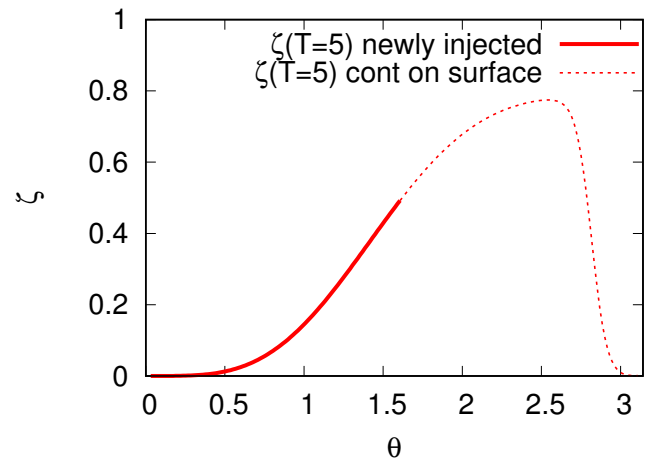

(c)

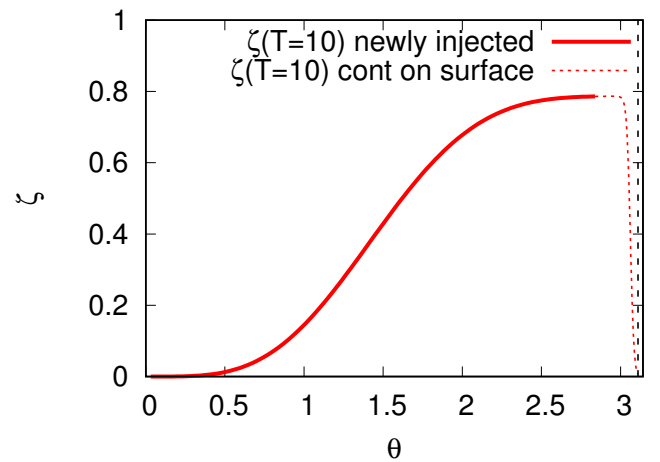

Figure 3: (a) The extent of diffusion $\zeta$ vs angular position $\theta$ for (a) time $T=1$, (b) time $T=5$ and (c) time $T=10$. In (a) the prediction is compared against a very early-time approximation for $\zeta$ (equation (37)) which corresponds to a spherically symmetric solute concentration field as would be found in a rigid drop. In both (b) and (c), the $\theta$ domain is divided into material points newly injected onto the surface since time zero $\left(\theta_{0}<\theta_{\mathrm{i}}\right)$, and material points continually on the surface throughout the evolution $\left(\theta_{0}>\theta_{\mathrm{i}}\right)$. Here $\theta_{0}$ is the initial location (found via equation (9)) and the injection point is $\theta_{\mathrm{i}}=\frac{\pi}{100}$. 
(a)

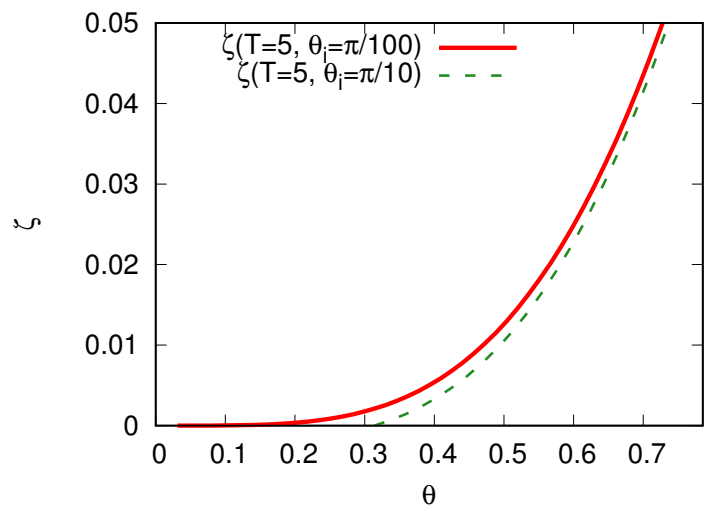

(b)

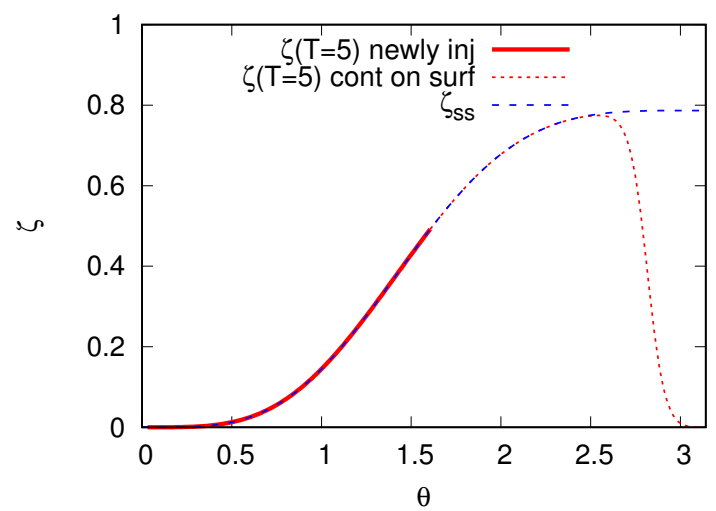

Figure 4: (a) The extent of diffusion $\zeta$ vs angular position $\theta$ plotted over a restricted domain $\theta<\frac{\pi}{4}$ at time $T=5$ and with two different values of $\theta_{\mathrm{i}}\left(\theta_{\mathrm{i}}=\frac{\pi}{10}\right.$ and $\left.\theta_{\mathrm{i}}=\frac{\pi}{100}\right)$. (b) $\zeta$ vs $\theta$ again for $T=5$ but plotted now for a wider domain of $\theta$ values, and with $\theta_{\mathrm{i}}=\frac{\pi}{100}$. A distinction is now made between material points that are newly injected since time zero and those that have been continually on the surface. Moreover these data for $T=5$ are compared with a steady state formula $\zeta_{\mathrm{ss}}$ vs $\theta$. 
(a)

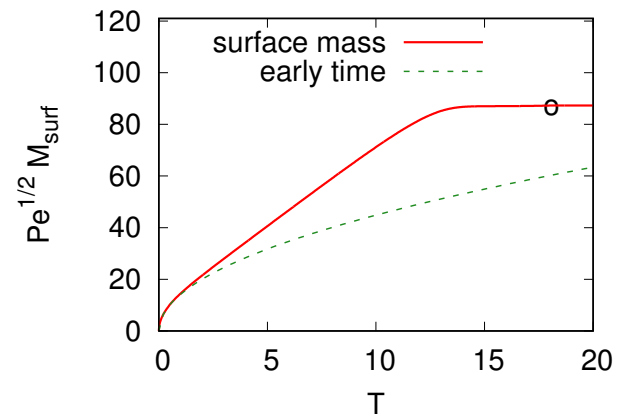

(b)

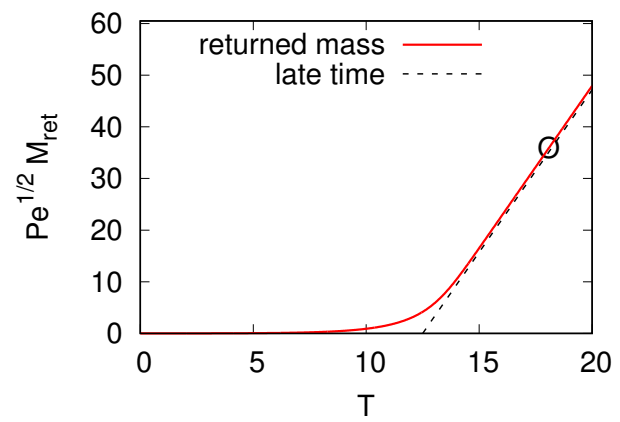

Figure 5: (a) The amount of solute mass $P e^{1 / 2} M_{\text {surf }}$ accumulated near the drop surface vs time $T$, as predicted by boundary layer theory. For comparison, the near surface mass in the very early-time limit is shown: this early-time formula assumes mass transfer as per a rigid drop. In the limit of later times the $P e^{1 / 2} M_{\text {surf }}$ from boundary layer theory attains an exact steady state (indicated by o). (b) The solute mass $P e^{1 / 2} M_{\text {ret }}$ returned to the interior of the drop vs time $T$. The return point $\theta_{\mathrm{r}}$ is chosen as $\frac{99 \pi}{100}$ here. After a certain time (indicated by $\circ$ ), $P e^{1 / 2} M_{\text {ret }}$ is known to attain an exactly steady rate of growth. To guide the eye, this late-time asymptotic growth has also been extrapolated back to the $T$ axis. 
(a)

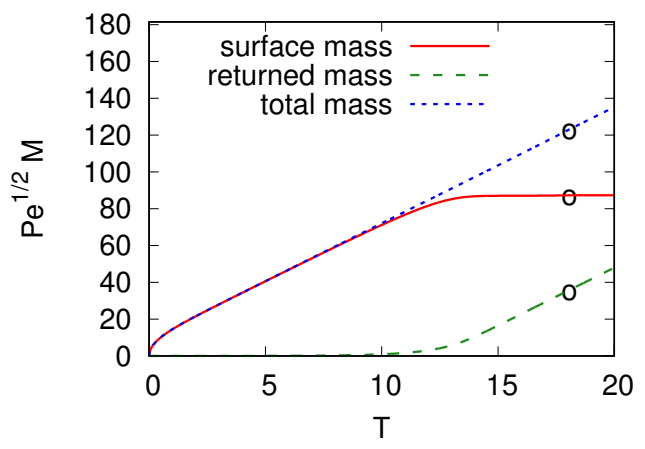

(b)

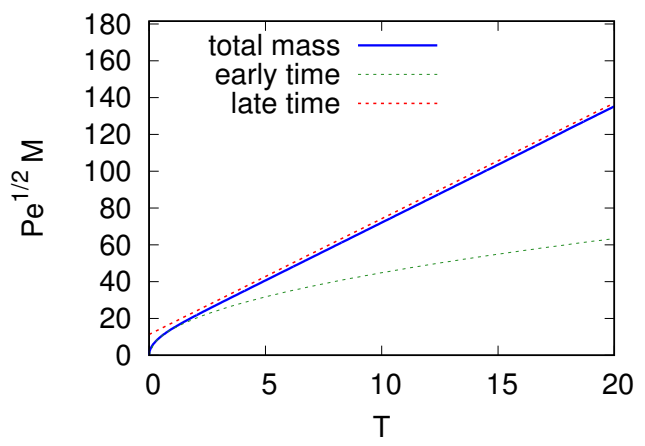

(c)

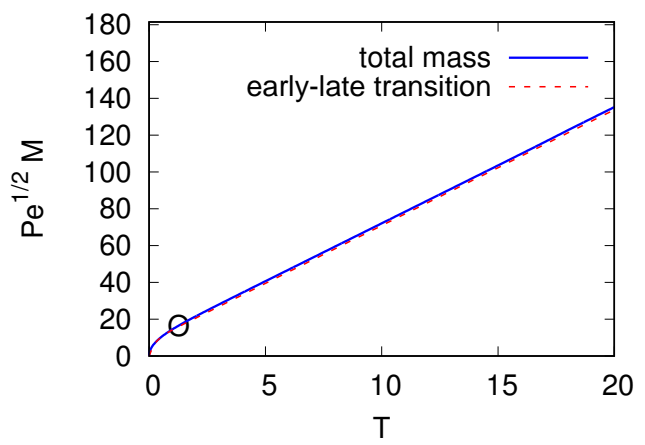

Figure 6: (a) The solute mass $P e^{1 / 2} M$ in the drop (as predicted by boundary layer theory) divided into two components, the mass accumulated adjacent to the surface $P e^{1 / 2} M_{\text {surf }}$ and the mass returned to the interior of the drop $P e^{1 / 2} M_{\text {ret }}$. These data correspond to an injection point and a return point respectively $\theta_{\mathrm{i}}=\frac{\pi}{100}$ and $\theta_{\mathrm{r}}=\frac{99 \pi}{100}$. Given these $\theta_{\mathrm{i}}$ and $\theta_{\mathrm{r}}$ values, the symbol $\circ$ indicates the time at which $P e^{1 / 2} M_{\text {surf }}$ achieves a final steady state, and $P e^{1 / 2} M_{\text {ret }}$ achieves a final steady rate of growth. (b) $P e^{1 / 2} M$ compared with an early-time formula (a square root growth law, corresponding to a rigid drop) and a late-time formula (a final steady rate of mass increase). (c) $P e^{1 / 2} M$ compared to an approximation in which an abrupt transition from an early-time square root behaviour to a later time straight line behaviour is considered to occur at the time for which the slope of the square root matches that of the straight line. The transition point is denoted $\circ$. 


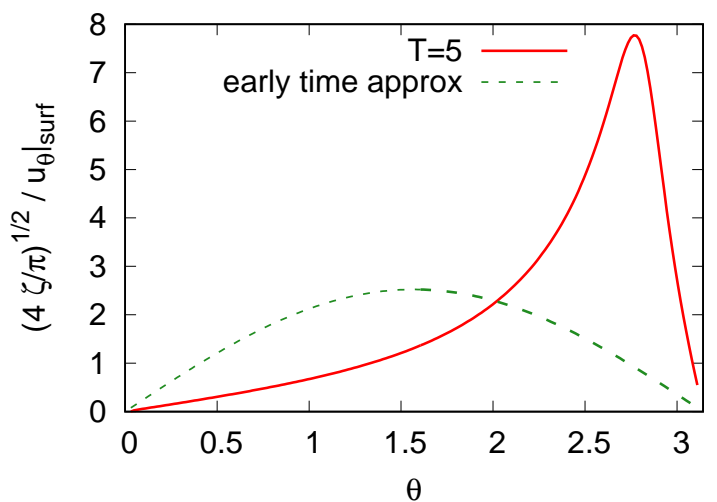

Figure 7: Mass distribution within the drop when $T=5$ as predicted by boundary layer theory, the mass distribution being defined as $\sqrt{4 \zeta / \pi} /\left.u_{\theta}\right|_{\text {surf }}$ which is the integrand in the expression for $P e^{1 / 2} M_{\text {surf }}$ : see equation (D.8). For comparison we also show the distribution, $\sqrt{4 T / \pi} \sin \theta$, which is what arises when the very early-time "rigid drop" approximation for $\zeta$ given by equation (37) is substituted into the integrand of equation (D.8) (although of course for $T=5$ that expression has been extrapolated well outside its early-time regime of validity). The $\sin \theta$ factor in this "rigid drop" expression arises from geometry: the drop has more surface area around its equator. 
(a)

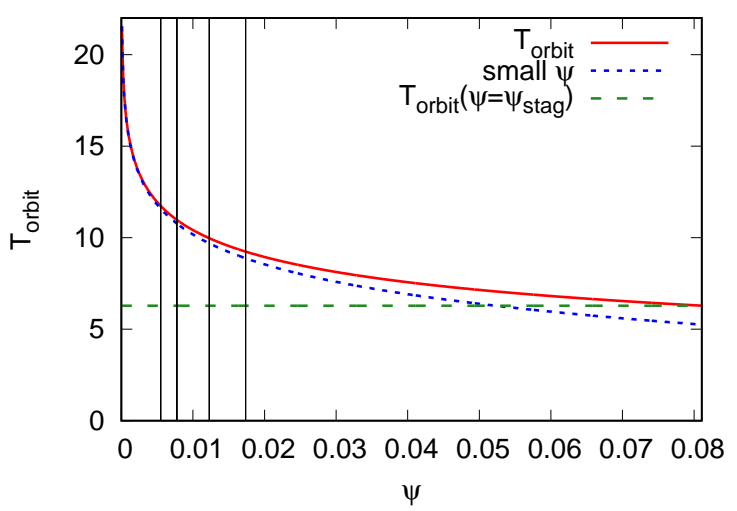

(b)

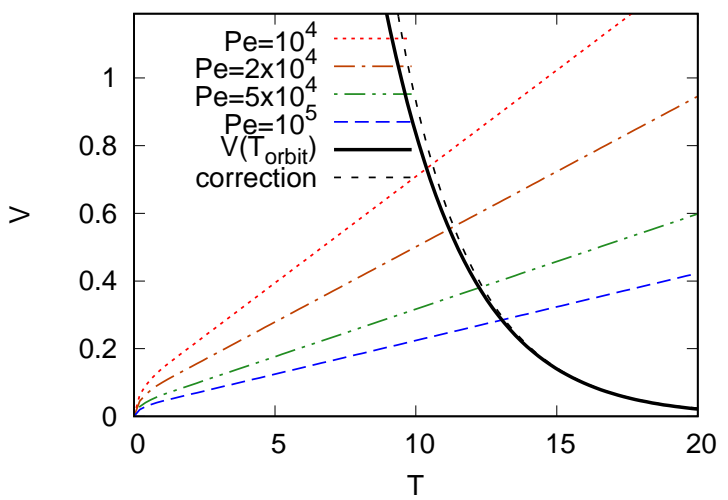

Figure 8: (a) The streamline orbit time $T_{\text {orbit }}$ as a function of streamfunction $\psi$, compared with a small $\psi$ asymptotic approximation (23). The horizontal line represents the time $T_{\text {orbit,stag }}$ required to orbit streamlines in the neighbourhood of the internal stagnation point $\psi_{\text {stag }}$. The vertical lines indicate the $\psi$ value to which solute has managed to invade the drop at the switching time when the streamlineaveraged formulation begins, respectively for $P e=100000$ (leftmost vertical line), 50000, 20000 and 10000 (rightmost vertical line). (b) The volume $V$ of the drop invaded by solute as a function of elapsed time $T$ (as predicted by boundary layer theory; see equation (D.9)) for various Peclet numbers $P e$. In addition the volume $V$ enclosed between the surface of the drop and a streamline with orbit time $T_{\text {orbit }}$. The intersections between the various curves represent, for each given $P e$, the instants at which sufficient time has elapsed that material has orbited once around the volume invaded, hence giving the switching time $T_{\text {switch }}$ from the boundary layer theory to the streamline-averaged one. For simplicity in locating these intersections we use an asymptotic approximation to $V$ vs $T_{\text {orbit }}$ (solid curve; equation (26)) valid in the limit of large $T_{\text {orbit }}$ (equivalent to the small $\psi$ formula in (a)). The correction associated with having finite $\psi$ (dashed curve adjacent to the solid curve in (b)) is small. 
(a)

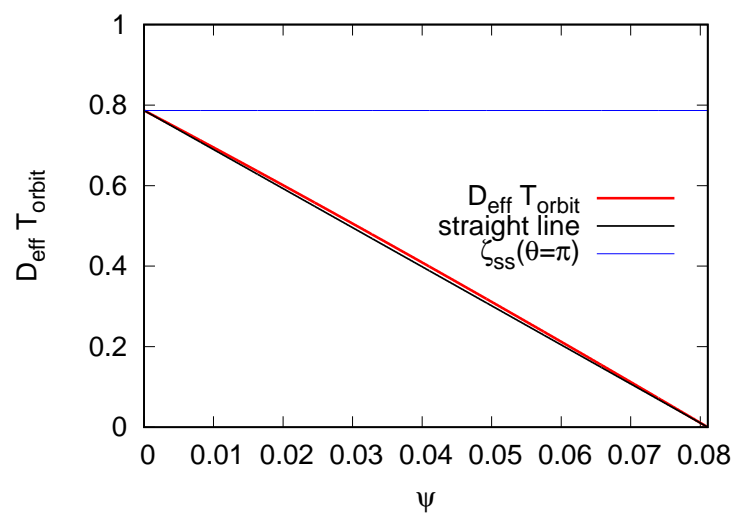

(b)

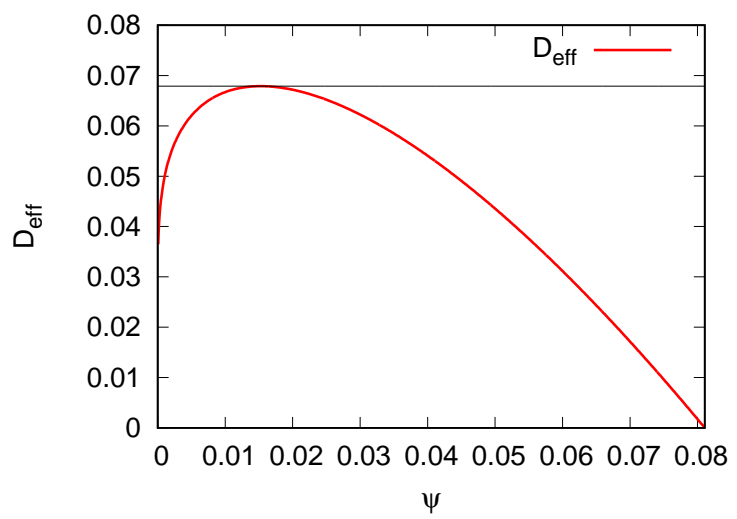

Figure 9: (a) The product $D_{\text {eff }} T_{\text {orbit }}$ vs streamfunction $\psi$. The function is very nearly a straight line (shown for comparison). Meanwhile the horizontal line is $\left.\zeta_{\mathrm{ss}}\right|_{\theta=\pi}$ as defined in equation (42). (b) $D_{\text {eff }}$ vs $\psi$. Note that $D_{\text {eff }}$ vanishes at both ends of the $\psi$ interval (for $\psi \rightarrow 0$ and for $\psi \rightarrow \psi_{\text {stag }}$ ) having a local maximum (see the horizontal line) in the interior of the interval. 
(a)

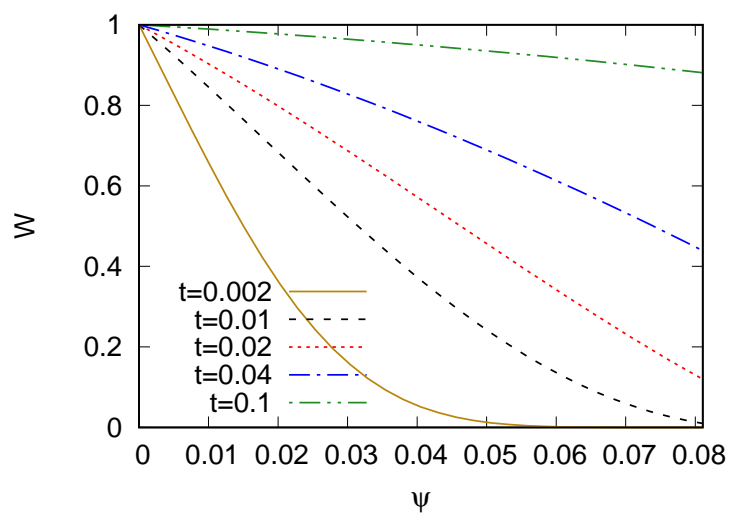

(b)

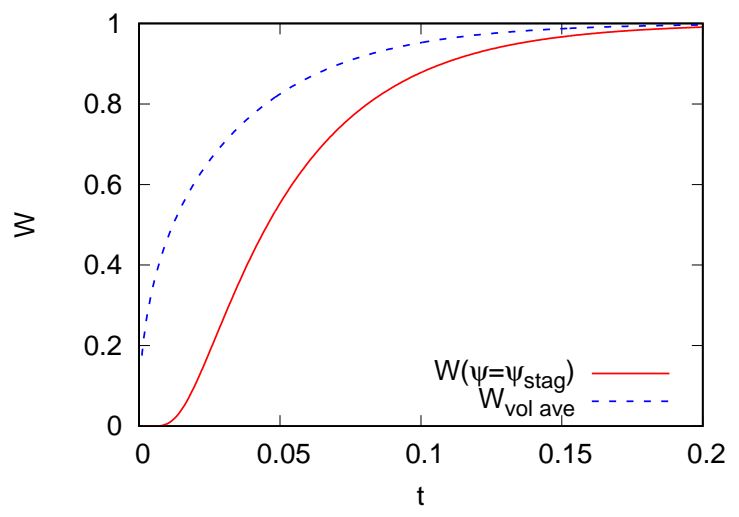

Figure 10: (a) Predictions of the streamline-averaged model for (normalised) solute mass fraction $W$ vs streamfunction $\psi$ at various times $t$. The data are formally for $P e=10000$, although the $P e$ value impacts negligibly upon the streamline-averaged solution itself, governing only the time at which the system first reaches the streamline-averaged state (which is well before any of the various times for which $W$ vs $\psi$ is plotted here). (b) $W$ vs time $t$ predicted by the streamline-averaged model, with $W$ taken either at the internal stagnation point $\psi=\psi_{\text {stag }}$ or else as a volume-average over the drop. These data again are formally for $P e=10000$ although, as before, the $P e$ value impacts very little on the solution: larger Peclet numbers would give an earlier switch into the streamline-averaged state, with less mass (and hence less volume-averaged solute mass fraction) having been transferred up to that point. 
(a)

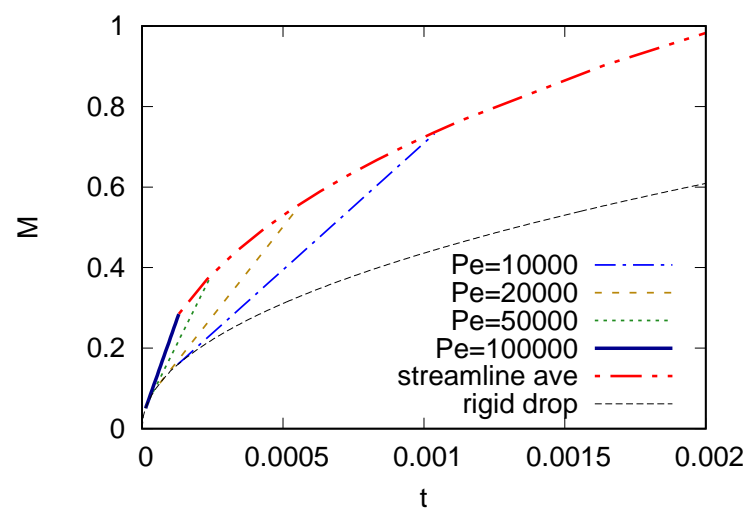

(b)

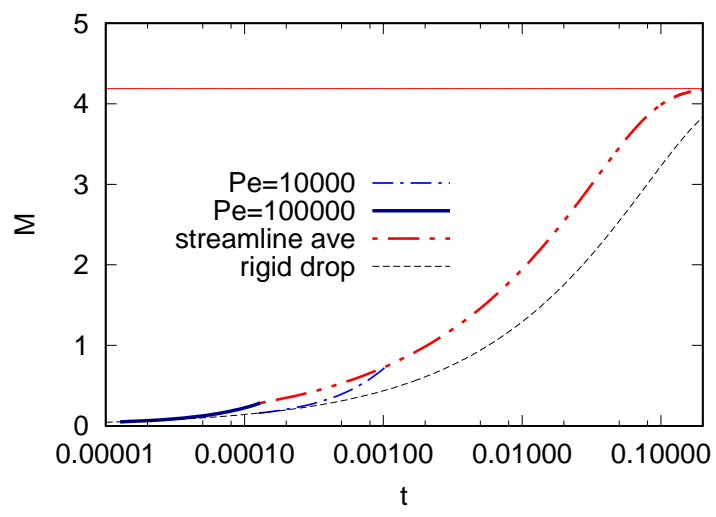

(c)

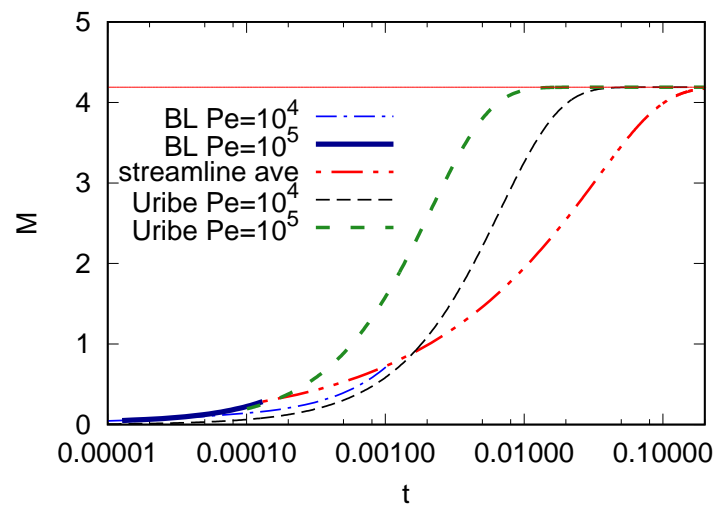

Figure 11: (a) Predictions of (normalised) solute mass $M$ in the drop vs time $t$ for various Peclet numbers $P e$, showing how for very small values of $t$, mass transfer crosses over from a boundary layer regime to a streamline-averaged regime. A comparison with the rigid drop prediction is also shown. (b) As per (a), but on a longer time scale, with a logarithmic axis for $t$. In this normalised or rescaled system, the final solute mass (horizontal line) is $\frac{4}{3} \pi$. (c) A comparison between the boundary layer theory developed in this work denoted 'BL' (again for various Peclet numbers), the streamline-averaged theory, and the previous boundary layer theory of Uribe-Ramirez and Korchinsky (given by equation (49), see Uribe-Ramirez and Korchinsky (2000b)). 


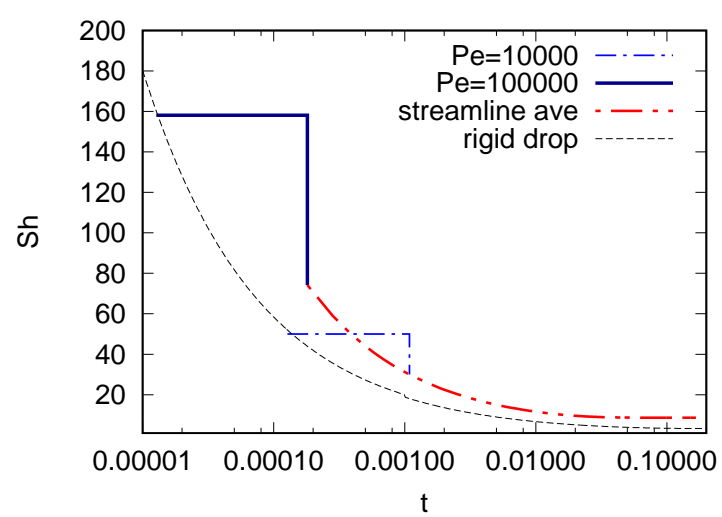

Figure 12: Predicted Sherwood number $S h$ vs dimensionless time $t$, for the boundary layer system at two different Peclet numbers $(P e=10000$ and $P e=100000)$ showing how $S h$ departs from the rigid drop case and subsequently undergoes a jump as the system switches into the streamline-averaged state. 